\title{
Right marker speeds of solutions to the KPP equation with noise
}

\author{
Sandra Kliem *
}

March 25, 2022

\begin{abstract}
We consider the one-dimensional KPP-equation driven by space-time white noise. We show that for all parameters above the critical value for survival, there exist stochastic wavelike solutions which travel with a deterministic positive linear speed. We further give a sufficient condition on the initial condition of a solution to attain this speed. Our approach is in the spirit of corresponding results for the nearest-neighbor contact process respectively oriented percolation. Here, the main difficulty arises from the moderate size of the parameter and the long range interaction. Stopping times and averaging techniques are used to overcome this difficulty.
\end{abstract}

Key words: stochastic PDE; KPP equation; white noise; travelling wave speed; near-critical. MSC2000 subject classification. Primary 60H15; Secondary 35R60.

\section{Introduction}

The Kolmogorov-Petrovskii-Piskunov-(KPP)-equation (also known as the Kolmogorov- or Fisher-equation) with noise is given as

$$
\partial_{t} u=\partial_{x x} u+\theta u-u^{2}+u^{\frac{1}{2}} d W, \quad t>0, x \in \mathbb{R}, \quad u(0, x)=u_{0}(x) \geq 0,
$$

where $W=W(t, x)$ is space-time white noise and $\theta>0$ a parameter. The deterministic part of this one-dimensional stochastic partial differential equation (SPDE) is, after appropriate scaling, a case of the well-studied KPP-equation. Note that by Mueller and Tribe [16, Lemma 2.1.2], constant front-factors in the PDEs/SPDEs to be referred to, can and will be changed without comment to fit into our framework. Including the noise term, one can think of $u(t, x)=u_{t}(x)=u_{t}^{\left(u_{0}\right)}(x)$ as the (random) density of a population in time and space. Leaving out the term $\theta u-u^{2}$, the above SPDE is the density of a superBrownian motion (cf. Perkins [18, Theorem III.4.2]), the latter being the high density limit of branching particle systems. The additional term of $\theta u$ models linear mass creation at rate $\theta>0,-u^{2}$ models death due to competition respectively overcrowding. In [17, Mueller and Tribe obtain solutions to (1.1) as (weak) limits of approximate densities of occupied sites in rescaled one-dimensional long range contact processes.

Let $\mathcal{C}^{+}$denote the space of non-negative continuous functions on $\mathbb{R}$. The existence and uniqueness in law of solutions to (1.1) in the space of non-negative continuous functions with slower than exponential growth $\mathcal{C}_{\text {tem }}^{+}$

$$
\mathcal{C}_{\text {tem }}^{+}=\left\{f \in \mathcal{C}^{+}:\|f\|_{\lambda}<\infty \text { for all } \lambda>0\right\} \text { with }\|f\|_{\lambda}=\sup _{x \in \mathbb{R}}|f(x)| e^{-\lambda|x|},
$$

\footnotetext{
*Institut für Mathematik, Goethe Universität, Frankfurt am Main, Germany. E-mail: kliem@math.uni-frankfurt.de
} 
is established in Tribe [19, Theorem 2.2]. Here, a solution to (1.1) is to be understood in the sense of a weak solution (see Notation 1.4 below). Denote with $\mathbb{P}_{u_{0}}$ the law of such a solution starting in $u_{0} \in \mathcal{C}_{\text {tem }}^{+}$. By [19, Theorem 2.2], the map $f \mapsto \mathbb{P}_{f}$ on $\mathcal{C}_{\text {tem }}^{+}$is continuous and the family of laws $\mathbb{P}_{f}, f \in \mathcal{C}_{\text {tem }}^{+}$ forms a strong Markov family. For $\nu \in \mathcal{P}\left(\mathcal{C}_{\text {tem }}^{+}\right)$, the space of probability measures on $\mathcal{C}_{\text {tem }}^{+}$, denote $\mathbb{P}_{\nu}(A)=\int_{\mathcal{C}_{\text {tem }}^{+}} \mathbb{P}_{f}(A) \nu(d f)$. Use $\mathbb{E}_{u_{0}}$ respectively $\mathbb{E}_{\nu}$ to denote respective expectations.

Let $\tau=\inf \{t \geq 0: u(t, \cdot) \equiv 0\}$ be the extinction-time of the process. By [16, Theorem 1], there exists a critical value $\theta_{c}>0$ such that for any initial condition $u_{0} \in \mathcal{C}_{c}^{+} \backslash\{0\}$ with compact support and $\theta<\theta_{c}$, the extinction-time of $u$ solving (1.1) is finite almost surely. For $\theta>\theta_{c}$, survival, that is $\tau=\infty$, happens with positive probability.

The investigation of the dynamics of solutions to (1.1) is a major challenge, where the main difficulty comes from the competition term $-u^{2}$. Without competition, the underlying additive property facilitates the use of Laplace functionals. Including competition, only subadditivity in the sense of [16, Lemma 2.1.7] respectively Kliem [14, Remark 2.1(i)] holds, that is, for $u_{0}, v_{0} \in \mathcal{C}_{\text {tem }}^{+}$and $w_{0} \equiv u_{0}+v_{0}$ there exists a coupling of solutions $\left(u_{t}\right)_{t \geq 0},\left(v_{t}\right)_{t \geq 0},\left(w_{t}\right)_{t \geq 0}$ to (1.1) with respective initial conditions $u_{0}, v_{0}, w_{0}$ such that $w_{t}(x) \leq u_{t}(x)+v_{t}(x)$ for all $t \geq 0, x \in \mathbb{R}$ almost surely.

Write $\langle f, g\rangle=\int f(x) g(x) d x$. For the process in (1.1) one has a self-duality relationship in the form

$$
\mathbb{E}_{u_{0}}\left[e^{-2<u(t), v_{0}>}\right]=\mathbb{E}_{u_{0}} \otimes \mathbb{E}_{v_{0}}\left[e^{-2\langle u(s), v(t-s)\rangle}\right]=\mathbb{E}_{v_{0}}\left[e^{-2<u_{0}, v(t)>}\right]
$$

for all $0 \leq s \leq t$ and $u_{0}, v_{0} \in \mathcal{C}_{t e m}^{+}$, where $u(t), v(t)$ are independent solutions to (1.1) with initial condition $u_{0}$ respectively $v_{0}$ (cf. [14, (2.1)]). Use $\mathcal{P}(E)$ to denote the space of probability measures on $E$. In [14, Remark 2.5] this self-duality is used to prove existence of a unique upper invariant distribution $\mu \in \mathcal{P}\left(\mathcal{C}_{\text {tem }}^{+}\right)$satisfying

$$
\lim _{t \rightarrow \infty} \inf \left\{\mathbb{E}_{\psi}\left[e^{-2<u(T+t), \phi>}\right] ; \psi \in \mathcal{C}_{t e m}^{+}\right\}=\int e^{-2<f, \phi>} \mu(d f)=\mathbb{P}_{\phi}(\tau<\infty)
$$

for all $T>0, \phi \in \mathcal{C}_{c}^{+}$. In [9, Theorem 1], Horridge and Tribe give sufficient conditions ("uniformly distributed in space") for initial conditions to be in the domain of attraction of $\mu$. They characterize $\mu$ by the right hand side of (1.4) and show that it is the unique translation invariant stationary distribution satisfying $\mu(\{f: f \not \equiv 0\})=1$. The result and method of proof are in the spirit of Harris' convergence theorem for additive particle systems (cf. Durrett [5, Theorem 3.3]).

Recall the construction of solutions to (1.1) from [17] by means of limits of densities of rescaled long range contact processes. When investigating solutions to the SPDE (1.1), it is only natural to anticipate and/or investigate behavior similar in spirit to the approximating systems. Indeed, 9] successfully applied the method of proof of Harris' convergence theorem for additive particle systems to prove a corresponding result in the context of SPDEs (1.1). Due to the long range interaction and the lack of a dual process, results for long range contact processes are limited. More is known for the nearest-neighbor contact process $\left(\xi_{t}\right)_{t \geq 0}$ on $\mathbb{Z}$ (cf. Griffeath [7]), where the neighborhood of a site $x \in \mathbb{Z}$ is restricted to $\{x-1, x+1\}$. For the nearest-neighbor contact process a full description of the limiting law of a solution is available. The limiting law is the weighted average of the Dirac-measure on the "all-unoccupied" configuration and the upper invariant measure of the process, $\nu$, where the weight on the former coincides with the extinction probability (see [7, Theorem 5]).

In what follows, let $S$ be the space of all subsets of $\mathbb{Z}$. By identifying the state of the process $\xi_{t}$ at time $t$ with the set of occupied sites, we can consider $\left(\xi_{t}\right)_{t \geq 0}$ as an $S$-valued process. Let $\lambda$ be the birth-parameter, the death-parameter is set to one. Set $\lambda_{c}=\sup \left\{\lambda \geq 0: \mathbb{P}\left(\tau^{\{0\}}=\infty\right)=0\right\}$, where $\tau^{\{0\}}=\inf \left\{t \geq 0: \xi_{t}^{\{0\}}=\emptyset\right\}$ is the extinction time of the population starting with zero being the only occupied site at time 0 .

The proof of complete convergence for the nearest-neighbor case relies in essence on the progression of the so-called edge processes $l_{t}^{A} \equiv \min \left\{x: x \in \xi_{t}^{A}\right\}, r_{t}^{A} \equiv \max \left\{x: x \in \xi_{t}^{A}\right\}, A \in S$ fixed. Due to the 
nearest-neighbor interaction one can easily show that

$$
\xi_{t}^{\{0\}}=\xi_{t}^{A} \cap\left[l_{t}^{\{0\}}, r_{t}^{\{0\}}\right]=\xi_{t}^{(-\infty, 0] \cap \mathbb{Z}} \cap \xi_{t}^{[0, \infty) \cap \mathbb{Z}} \text { for all } 0 \in A \subset \mathbb{Z} \text { on }\left\{\tau^{\{0\}}>t\right\}
$$

(cf. [7, Theorem 3]). Moreover,

$$
l_{t}^{\{0\}}=l_{t}^{[0, \infty) \cap \mathbb{Z}} \text { and } r_{t}^{\{0\}}=r_{t}^{(-\infty, 0] \cap \mathbb{Z}} \text { on }\left\{\tau^{\{0\}}>t\right\} .
$$

In [3, Theorem 1.4 and Section 4] respectively [4, Section 3, (8)-(9)], Durrett shows for the nearest-neigbor contact process respectively for oriented percolation in two dimensions that

$$
-\lim _{t \rightarrow \infty} \frac{l_{t}^{\{0\}}}{t}=\lim _{t \rightarrow \infty} \frac{r_{t}^{\{0\}}}{t}=\alpha \text { a.s., } \quad \text { where } \alpha \begin{cases}>0, & \text { if } \tau^{\{0\}}=\infty, \\ <0, & \text { if } \tau^{\{0\}}<\infty .\end{cases}
$$

In these models, edge speeds characterize critical values. Similar features were for instance recently observed in Bessonov and Durrett [2] for planar quadratic contact processes (here, two individuals are needed to produce a new one). Under long range interaction, (1.5)-(1.6) do not hold true any longer.

For these reasons, the study of the speed of the right (and thus by symmetry left) marker

$$
R_{0}(u(t)) \equiv R_{0}(t) \equiv \sup \{x \in \mathbb{R}: u(t, x)>0\} \quad \text { with } \sup \emptyset=-\infty
$$

of a solution to (1.1) starting in $u_{0} \in \mathcal{P}\left(\mathcal{C}_{\text {tem }}^{+}\right)$with $\mathbb{P}\left(R_{0}\left(u_{0}\right)<\infty\right)=1$ is of independent interest and yields new insights into the dynamics of solutions to (1.1). Note that $R_{0}(t)=-\infty$ if and only if $\tau \leq t$. Extending arguments of Iscoe [11] one can show that $R_{0}(u(0))<\infty$ implies $R_{0}(u(t))<\infty$ for all $t>0$. In [14, Remark 2.8], $\mathcal{C}_{\text {tem }}^{+}$-valued left- and right-upper measures were derived as analogues to the law of

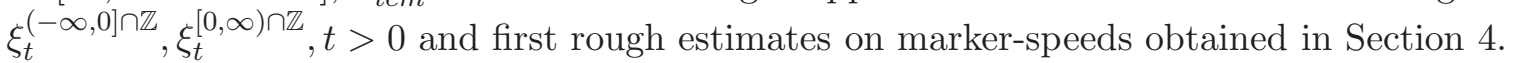

Let $R_{0}(t)$ as in (1.8). Using $R_{0}$ as a (right) wavefront marker, we look for so-called travelling wave solutions to (1.1), that is solutions with the properties

(i) $\quad R_{0}(u(t)) \in(-\infty, \infty)$ for all $t \geq 0$,

(ii) $u\left(t, \cdot+R_{0}(u(t))\right)$ is a stationary process in time.

Travelling wave solutions are of interest in models from physics, chemistry and biology (cf. Aronson and Weinberger [1]). In [19, the existence of travelling wave solutions for $\theta>\theta_{c}$ with non-negative wave speed, based on solutions to (1.1) with Heavyside initial data of the form $H_{0}(x) \equiv 1 \wedge(-x \vee 0)$ is established. In [19, Section 4] it is established that for $\theta>\theta_{c}$ any travelling wave solution has an asymptotic (possibly random) wave speed

$$
R_{0}(u(t)) / t \rightarrow A \in\left[0,2 \theta^{1 / 2}\right] \text { for } t \rightarrow \infty \text { almost surely. }
$$

It is further shown that for $\theta$ big enough, $A$ is close to $2 \theta^{1 / 2}$ with high probability. Strict positivity of $A$ remains an open problem if $\theta$ is of moderate size. Further open problems that arise are for instance if the wave-speed is deterministic or random, the dependence of the speed on the parameter $\theta$, the uniqueness of the distribution of the travelling waves and the shape of the wavefront. In this article, we make substantial progress to resolve the first two problems.

An alternative construction of travelling wave solutions is given in [14 in case $\theta>\theta_{c}$. The initial Heavyside-condition $H_{0}$ is replaced by an arbitrary non-negative continuous function $g_{0} \in \mathcal{C}_{c}^{+}$with compact support. As extinction (that is $\tau=\inf \left\{t \geq 0: u_{t} \equiv 0\right\}=\inf \left\{t \geq 0:\left\langle u_{t}, 1\right\rangle=0\right\}<\infty$ ) happens with probability $0<\mathbb{P}_{g_{0}}(\tau<\infty)<1$, we condition on non-extinction to obtain well-defined travelling wave solutions $\nu^{\left(g_{0}\right)}$. Note that $\nu^{\left(g_{0}\right)}$ denotes any subsequential limit obtained by this construction. The uniqueness of the limiting distribution remained as an open problem. 
Recall the discussion below (1.8). For $T>0$, denote by $v_{T}$ the left-upper measure on $\mathcal{C}_{\text {tem }}^{+}$correspond-

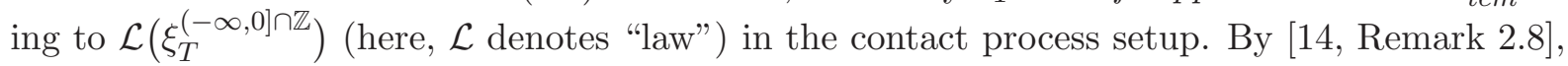

$$
\int e^{-2\langle f, g\rangle} v_{T}(d f)=\mathbb{P}\left(\left\langle\mathbb{1}_{(-\infty, 0)}(\cdot), u_{T}^{(g)}\right\rangle=0\right), \quad \text { for } g \in \mathcal{C}_{\text {tem }}^{+}
$$

Furthermore, for $u_{0} \in \mathcal{C}_{\text {tem }}^{+}$with $R_{0}\left(u_{0}\right) \leq 0$ and $T>0$ arbitrarily fixed one obtains the existence of a coupling with a random continuous process $\left(u_{T+t}^{*, l}\right)_{t \geq 0}$ with values in $\mathcal{C}_{\text {tem }}^{+}$such that

$$
u_{T+t}^{\left(u_{0}\right)}(x) \leq u_{T+t}^{*, l}(x) \quad \text { for all } x \in \mathbb{R}, t \geq 0 \text { almost surely, }
$$

where $\mathcal{L}\left(\left(u_{T+t}^{*, l}\right)_{t \geq 0}\right)=\mathbb{P}_{v_{T}}$ holds. Note in particular that such a coupling yields

$$
R_{0}\left(u_{T+t}^{\left(u_{0}\right)}\right) \leq R_{0}\left(u_{T+t}^{*, l}\right) \text { for all } t \geq 0 \text { almost surely. }
$$

By symmetry, analogous results hold for a right-upper measure, say $\kappa_{T}$, where we make use of the notations $L_{0}(f) \equiv \inf \{x \in \mathbb{R}: f(x)>0\}$ and $u_{T+t}^{*, r}$ instead. In the appendix (cf. (5.8)) we indicate how to modify the techniques of [14] to construct travelling wave solutions $\nu^{*, l}$ respectively $\nu^{*, r}$ from $u^{*, l}$ respectively $u^{*, r}$.

The first main result of this article is a pre-step to a result in the spirit of the first case of (1.7), where the almost sure convergence is replaced by $\mathcal{L}^{1}$-convergence.

Proposition 1.1. For all $\theta>\theta_{c}$, the limit $B \equiv B(\theta) \equiv \lim _{t \rightarrow \infty} \mathbb{E}\left[R_{0}\left(u_{t}^{*, l}\right)\right] / t$ exists and is strictly positive. Moreover, for all $\theta_{c}<\underline{\theta} \leq \theta_{1} \leq \theta_{2} \leq \bar{\theta}$, there exists a constant $C=C(\underline{\theta}, \bar{\theta})$ such that

$$
B\left(\theta_{2}\right)-B\left(\theta_{1}\right) \geq C\left(\theta_{2}-\theta_{1}\right) .
$$

We note that the strict positivity of $B(\theta)$ follows from (1.14), once $B(\theta) \geq 0$ is established for all $\theta>\theta_{c}$. Our approach relies on establishing the estimate (1.14) along the lines of the corresponding result for contact processes in [3, Lemma 4.2].

Recall from above that $H_{0}$ denotes Heavyside initial data of the form $H_{0}(x) \equiv 1 \wedge(-x \vee 0)$.

Definition 1.2. Let

$$
\mathcal{H}=\left\{f \in \mathcal{C}_{\text {tem }}^{+}: \exists x_{0} \in \mathbb{R}, \epsilon>0: f(x) \geq \epsilon H_{0}\left(x-x_{0}\right) \text { for all } x \in \mathbb{R}\right\}
$$

and $\mathcal{H}^{R}=\left\{f \in \mathcal{H}: R_{0}(f) \in \mathbb{R}\right\}$.

Our second main result concerns the limiting speeds of several right markers. It establishes in particular the existence of at least one travelling wave with positive deterministic speed.

Theorem 1.3. Let $\theta>\theta_{c}$. Then

$$
R_{0}\left(u_{T}^{*, l}\right) / T \rightarrow B \text { as } T \rightarrow \infty \text { almost surely and in } \mathcal{L}^{1} .
$$

For any travelling wave solution $\nu^{*, l}$,

$$
R_{0}\left(u_{T}^{\left(\nu^{*, l}\right)}\right) / T \rightarrow B \text { almost surely as } T \rightarrow \infty
$$

and $\left(0 \vee R_{0}\left(u_{T}^{\left(\nu^{*, l}\right)}\right)\right) / T \rightarrow B$ in $\mathcal{L}^{1}$.

For initial conditions $\psi \in \mathcal{H}^{R}$,

$$
R_{0}\left(u_{T}^{(\psi)}\right) / T \rightarrow B \text { as } T \rightarrow \infty \text { in probability and in } \mathcal{L}^{1} .
$$

For any travelling wave solution $\nu^{(\psi)}$,

$$
R_{0}\left(u_{T}^{\left(\nu^{(\psi)}\right)}\right) / T \rightarrow B \text { almost surely as } T \rightarrow \infty
$$

and $\left(0 \vee R_{0}\left(u_{T}^{\left(\nu^{(\psi)}\right)}\right)\right) / T \rightarrow B$ in $\mathcal{L}^{1}$. 
Recall (1.7). It remains to prove, for instance, that for $\theta<\theta_{c},\left(R_{0}\left(u_{T}^{*, l}\right) \vee 0\right) / T$ converges (in some sense) to 0 . In combination with (1.16) this would then show that the edge speeds of solutions starting in left- or right-upper measures characterize critical values. This is work in progress.

For the remainder, let us recall some notation and Theorem 2.2 from [19] that are often used in the present article.

Notation 1.4 (Notation from [19], also see Subsection 1.2 of [14]). 1. Equip $\mathcal{C}_{\text {tem }}^{+}$with the topology given by the norms $\|f\|_{\lambda}$ for $\lambda>0$. Note that $d(f, g) \equiv \sum_{n \in \mathbb{N}}\left(1 \wedge\|f-g\|_{1 / n}\right)$ metrizes this topology and makes $\mathcal{C}_{\text {tem }}^{+}$a Polish space. Let $\left(\mathcal{C}\left([0, \infty), \mathcal{C}_{\text {tem }}^{+}\right), \mathcal{U}, \mathcal{U}_{t}, U(t)\right)$ be continuous path space, the canonical right continuous filtration and the coordinate variables.

2. In [19, (2.4)-(2.5)], the more general equation

$$
\partial_{t} u=\partial_{x x} u+\alpha+\theta u-\beta u-\gamma u^{2}+u^{\frac{1}{2}} d W, \quad t>0, x \in \mathbb{R}, \quad u(0, x)=u_{0}(x) \geq 0
$$

with $\alpha, \beta, \gamma \in \mathcal{C}\left([0, \infty), \mathcal{C}_{\text {tem }}^{+}\right)$is under consideration. We may interpret $\alpha$ as the immigration rate, $\theta-\beta$ as the mass creation-annihilation rate and $\gamma$ as the overcrowding rate.

$A$ solution to (1.20) consists of a filtered probability space $\left(\Omega, \mathcal{F}, \mathcal{F}_{t}, \mathbb{P}\right)$, an adapted white noise $W$ and an adapted continuous $\mathcal{C}_{\text {tem }}^{+}$valued process $u(t)$ such that for all $\phi \in \mathcal{C}_{c}^{\infty}$, the space of infinitely differentiable functions on $\mathbb{R}$ with compact support,

$$
\begin{aligned}
\langle u(t), \phi\rangle= & \langle u(0), \phi\rangle+\int_{0}^{t}\left\langle u(s), \phi_{x x}+(\theta-\beta(s)-\gamma(s) u(s)) \phi\right\rangle d s \\
& +\int_{0}^{t}\langle\alpha(s), \phi\rangle d s+\int_{0}^{t} \int|u(s, x)|^{1 / 2} \phi(x) d W_{x, s} .
\end{aligned}
$$

If in addition $\mathbb{P}(u(0, x)=f(x))=1$ then we say the solution $u$ starts at $f$.

Theorem 1.5 (Theorem 2.2a)-b) of [19]).

a) For all $f \in \mathcal{C}_{\text {tem }}^{+}$there is a solution to (1.20) started at $f$.

b) All solutions to (1.20) started at $f$ have the same law which we denote by $Q^{f, \alpha, \beta, \gamma}$. The map $(f, \alpha, \beta, \gamma) \rightarrow Q^{f, \alpha, \beta, \gamma}$ is continuous. The laws $Q^{f, \alpha, \beta, \gamma}$ for $f \in \mathcal{C}_{\text {tem }}^{+}$form a strong Markov family.

c) For $R, T>0$ let $\mathcal{U}_{R, T}=\sigma(U(t, x): t \leq T,|x| \leq R)$. Then the two laws $Q^{f, \alpha, \beta, \gamma}, Q^{f, \alpha, 0,0}$ are mutually absolutely continuous on $\mathcal{U}_{R, T}$.

Note that Tribe [19] later uses the notation $Q^{f} \equiv Q^{f, 0,0,1}$ where we use $\mathbb{P}_{f}$. Also, when the parameter $\theta$ in (1.20) is not clear from the context, we write $Q^{f, \alpha, \beta, \gamma}(\theta)$.

Finally, let $\stackrel{\mathcal{D}}{=}$ denote equality in distribution. Constants may change from line to line. We drop $\theta$ if the context is clear.

Outline. The paper is organized as follows. Sections 23 are dedicated to the proof of Proposition 1.1 . that is, the positivity of $B(\theta)$ for all $\theta>\theta_{c}$. In Subsections 2.1 2.3 the groundwork is laid for the proof of Proposition 1.1. In Subsection 2.2 we already state the estimate that lies at the heart of the proof of Proposition 1.1, see Proposition 2.10, Its proof follows in Subsection 2.4. A substantial part of the proof goes into an estimate on the gain of mass at the front due to an increase in $\theta$, see Proposition 2.17. We therefore postpone the proof of the latter to Section 3 ,

Section 4 is dedicated to the proof of Theorem 1.3. that is, the convergence of the linear speed of right markers to $B(\theta)$. In the appendix, Section 5 , the construction of travelling wave solutions from [14] is extended to include $\nu^{*, l}$ and $\nu^{(\psi)}$ with initial conditions $\psi \in \mathcal{H}^{R}$ (cf. (1.15) and below). Coupling techniques that are often used are summarized for reference. 


\section{Preliminary results}

\subsection{The terms under investigation}

Let $\theta>\theta_{c}$ be arbitrarily fixed. Recall the sequence of laws $\left(v_{T}\right)_{T>0}$ on $\mathcal{C}_{\text {tem }}^{+}$and $\left(u_{T+t}^{*, l}\right)_{t \geq 0}$ for $T>0$ fixed satisfying $\mathcal{L}\left(u_{T+t}^{*, l}\right)=v_{T+t}$ from (1.11)-(1.12). Note that $v .=v \cdot(\theta)$ and $u_{T+.}^{*, l}=u_{T+\cdot}^{*, l}(\theta)$. From [14, Corollary 4.7 and Notation 1.3-4.] we conclude that the double-integrals below are well-defined with values in $[-\infty, \infty)$. Let

$$
\alpha_{T}(\theta)=\alpha_{T}=\frac{2}{T} \int_{0}^{T / 2} \mathbb{E}\left[R_{0}\left(u_{T / 2+s}^{*, l}\right)\right] d s
$$

In fact, $\mathbb{E}\left[R_{0}\left(u_{T}^{*, l}\right)\right] / T$ and $\alpha_{T} / T$ are uniformly bounded in $T \geq 1$ as we conclude from [14, Corollary 4.7 ] and the next lemma.

\subsection{Estimates on right-markers}

Note that in this subsection, for all $\theta_{c}<\underline{\theta} \leq \theta \leq \bar{\theta}$ the constants to follow only depend on $\theta$ through $\underline{\theta}, \bar{\theta}$.

Lemma 2.1. For all $u_{0} \in \mathcal{H}$, there exists a constant $C=C\left(u_{0}\right)>0$ such that

$$
\mathbb{E}_{u_{0}}\left[0 \vee\left(-R_{0}\left(u_{t}\right)\right)\right] \leq C(1+t)
$$

holds uniformly in $t \geq 0$. Moreover, there exist $C_{i}=C_{i}\left(u_{0}\right)>0, i=1,2$ such that for all $M>0$,

$$
\mathbb{E}_{u_{0}}\left[\frac{-R_{0}\left(u_{t}\right)}{t} \mathbb{1}_{\left\{R_{0}\left(u_{t}\right)<-M t\right.}\right] \leq C_{1} e^{-C_{2} M}
$$

holds uniformly in $t \geq 1$.

Proof. Let $u_{0} \in \mathcal{H}$. Recall $\epsilon, x_{0}$ from the definition of $\mathcal{H}$. By domination, that is using [19, Lemma 3.1b)], we assume without loss of generality that $u_{0}=\epsilon H_{0}\left(\cdot-x_{0}\right)$. We further assume $x_{0}=0$ by the shift invariance of the dynamics.

We reason as in the proof of [19, Lemma 3.5]. The author uses the wave-marker $R_{1}(t)=\ln \left(\left\langle e^{*}, u_{t}\right\rangle\right)$ and Heavyside initial data $H_{0}$ instead. It is shown that there exist $c=c(\theta), a=a(\theta), \delta=\delta(\theta)>0$ such that $\mathbb{P}_{H_{0}}\left(R_{1}(t) \leq-a-c m t\right) \leq(1-\delta / 4)^{m}$ for all $t \geq 0, m \in \mathbb{N}$.

We claim that this holds for $H_{0}$ replaced by $u_{0}=\epsilon H_{0}, R_{1}(t)$ replaced by $R_{0}(t)$ and $a$ replaced by 0 as well. Moreover, the constants $c, \delta$ only depend on $\epsilon, \underline{\theta}$ and $\bar{\theta}$. Indeed, replace $a>0$ by $a=0$. Reason as in the given proof with $R_{1}(f)$ replaced by $R_{0}(f)$ and $\psi_{0}$ replaced by $\psi_{0}^{\prime} \equiv \epsilon \psi_{0}$ until the last set of equations. Choose $r=c t$ for $t \geq 1$ arbitrarily fixed and $r=c$ for $t \in[0,1$ ) (and thus $Q^{\psi_{0}}\left(T_{0}(U) \leq t\right) \leq Q^{\psi_{0}}\left(T_{0}(U) \leq 1\right) \leq \delta / 4$ in the notation of [19]). In the last set of equations, use that for a superprocess with initial symmetric condition $\psi_{0}^{\prime}$ and law $\overline{\mathbb{P}}_{\psi_{0}^{\prime}}$, there exists $\delta>0$ small enough such that $\overline{\mathbb{P}}_{\psi_{0}^{\prime}}\left(R_{0}\left(u_{t}\right) \geq 0\right) \geq \overline{\mathbb{P}}_{\psi_{0}^{\prime}}(\tau>t) / 2 \geq \mathbb{P}_{\psi_{0}^{\prime}}\left(\left\langle u_{t}, 1\right\rangle \geq \delta\right) \geq \delta / 2$ to obtain for all $t \geq 1$,

$$
\mathbb{P}_{u_{0}}\left(R_{0}(t) \leq-c m t\right) \leq(1-\delta / 4)^{m} \text { for all } m \in \mathbb{N} .
$$

As a result,

$$
\mathbb{E}_{u_{0}}\left[0 \vee\left(-R_{0}\left(u_{t}\right)\right)\right] \leq c t+\sum_{m \in \mathbb{N}}(1-\delta / 4)^{m} c(m+1) t \leq C(c, \delta) t
$$

For $t \in[0,1)$, the different choice of $r$ yields

$$
\mathbb{P}_{u_{0}}\left(R_{0}(t) \leq-c m\right) \leq(1-\delta / 4)^{m} \text { for all } m \in \mathbb{N}
$$


and

$$
\mathbb{E}_{u_{0}}\left[0 \vee\left(-R_{0}\left(u_{t}\right)\right)\right] \leq c+\sum_{m \in \mathbb{N}}(1-\delta / 4)^{m} c(m+1) \leq C(c, \delta)
$$

instead.

By $\lfloor x\rfloor$ we denote the greatest integer that is less than or equal to $x \in \mathbb{R}$. To obtain the second claim, for $0<M<c$ choose $C_{1}$ big enough and $C_{2}$ small enough such that $C(c, \delta) \leq C_{1} e^{-C_{2} c}$. For $M \geq c$ and $t \geq 1$

$$
\begin{aligned}
\mathbb{E}_{u_{0}}\left[\frac{-R_{0}\left(u_{t}\right)}{t} \mathbb{1}_{\left\{R_{0}\left(u_{t}\right)<-M t\right\}}\right] & \leq \sum_{m=\lfloor M / c\rfloor}^{\infty}(1-\delta / 4)^{m} c(m+1) \\
& =e^{\ln (1-\delta / 4)\lfloor M / c\rfloor} \sum_{m=0}^{\infty}(1-\delta / 4)^{m} c(m+1+\lfloor M / c\rfloor) \leq C_{1} e^{-C_{2} M}
\end{aligned}
$$

for $C_{1}=C_{1}(c, \delta)$ big enough and $C_{2}=C_{2}(c, \delta)$ small enough.

Corollary 2.2. There exists a constant $C>0$ such that

$$
\mathbb{E}\left[0 \vee\left(-R_{0}\left(u_{t}^{*, l}\right)\right)\right] \leq C(1+t)
$$

holds uniformly in $t>0$. Moreover, there exist $C_{i}>0, i=1,2$ such that for all $M>0$,

$$
\mathbb{E}\left[\frac{-R_{0}\left(u_{t}^{*, l}\right)}{t} \mathbb{1}_{\left\{R_{0}\left(u_{t}^{*, l}\right)<-M t\right\}}\right] \leq C_{1} e^{-C_{2} M}
$$

holds uniformly in $t \geq 1$.

Proof. The result follows again by domination, this time using (1.13) and $u_{0} \in \mathcal{H}^{R}$ with $R_{0}\left(u_{0}\right) \leq 0$ arbitrary.

Corollary 2.3. $\mathbb{E}_{u_{0}}\left[\left|R_{0}\left(u_{T}\right)\right|\right] / T, u_{0} \in \mathcal{H}^{R}$ and $\mathbb{E}\left[\left|R_{0}\left(u_{T}^{*, l}\right)\right|\right] / T$ are uniformly bounded in $T \geq 1$ (constants may depend on $\left.u_{0}\right)$.

Proof. Combine Lemma 2.1 respectively Corollary 2.2 with [14, Lemma 4.6].

Corollary 2.4. There exists a constant $C>0$ such that $\mathbb{E}\left[\left|R_{0}\left(u_{s}^{*, l}\right)\right|\right] \leq C$ for all $0<s \leq 1$. Moreover, for every $u_{0} \in \mathcal{H}^{R}$ there exists a constant $C\left(u_{0}\right)>0$ such that $\mathbb{E}\left[\left|R_{0}\left(u_{s}\right)\right|\right] \leq C\left(u_{0}\right)$ for all $0 \leq s \leq 1$.

Proof. Fix $0<s \leq 1$. Then $\mathbb{E}\left[0 \vee R_{0}\left(u_{s}^{*, l}\right)\right] \leq C$ follows from [14, Proposition 4.5] and $\mathbb{E}[0 \vee(-$ $\left.\left.R_{0}\left(u_{s}^{*, l}\right)\right)\right] \leq C$ from Corollary 2.2. For $u_{0} \in \mathcal{H}^{R}$, the bound for the positive part follows by domination and shift invariance, that is, $\mathbb{E}\left[0 \vee R_{0}\left(u_{s}\right)\right] \leq\left|R_{0}\left(u_{0}\right)\right|+\mathbb{E}\left[0 \vee R_{0}\left(u_{s}^{*, l}\right)\right] \leq\left|R_{0}\left(u_{0}\right)\right|+C=C\left(u_{0}\right)$. The bound for the negative part follows from Lemma 2.1 .

Corollary 2.5. $\alpha_{T} / T$ is uniformly bounded in $T \geq 1$.

Remark 2.6. The definition of the marker $R_{0}$ together with Corollaries 2.3 2.4 yields inf $\left\{t>0: u_{t}^{*, l} \equiv\right.$ $0\}=+\infty$ a.s., that is, the process $u^{*, l}$ does not die out in finite time. Thus, if we consider $u^{*, l}$, we do not have to bother with conditioning on non-extinction.

The existence of the following limit will turn out to be crucial in the following chapters. Non-negativity of the limit follows below. 
Lemma 2.7. The limit

$$
B=B(\theta)=\lim _{T \rightarrow \infty} \frac{\mathbb{E}\left[R_{0}\left(u_{T}^{*, l}\right)\right]}{T}=\inf _{T \geq 1} \frac{\mathbb{E}\left[R_{0}\left(u_{T}^{*, l}\right)\right]}{T} \in(-\infty, \infty)
$$

exists.

Proof. We work with $\mathbb{P}_{v_{1}}$, that is randomize the initial condition according to the law of $u_{1}^{*, l} \in \mathcal{C}_{\text {tem }}^{+}$. By the strong Markov property of the process we have for arbitrary $1 \leq s, t$,

$$
\begin{aligned}
\mathbb{E}\left[R_{0}\left(u_{s+t}^{*, l}\right)\right] & =\mathbb{E}\left[\mathbb{E}\left[R_{0}\left(u_{s+t}^{*, l}\right) \mid \mathcal{F}_{t}\right]\right]=\mathbb{E}\left[R_{0}\left(u_{s}^{\left(u_{t}^{*, l}\right)}\right)\right] \\
& =\mathbb{E}\left[R_{0}\left(u_{s}^{\left(u_{t}^{*, l}\left(\cdot+R_{0}\left(u_{t}^{*, l}\right)\right)\right)}\right)\right]+\mathbb{E}\left[R_{0}\left(u_{t}^{*, l}\right)\right] .
\end{aligned}
$$

Use monotonicity, that is (1.13) to further obtain

$$
\mathbb{E}\left[R_{0}\left(u_{s+t}^{*, l}\right)\right] \leq \mathbb{E}\left[R_{0}\left(u_{s}^{*, l}\right)\right]+\mathbb{E}\left[R_{0}\left(u_{t}^{*, l}\right)\right] .
$$

By subadditivity (cf. for instance Liggett [15, Theorem B22]) and from the uniform boundedness of $\mathbb{E}\left[\left|R_{0}\left(u_{T}^{*, l}\right)\right|\right] / T$ in $T \geq 1$, we conclude,

$$
\lim _{T \rightarrow \infty} \frac{\mathbb{E}\left[R_{0}\left(u_{T}^{*, l}\right)\right]}{T}=\inf _{T>0} \frac{\mathbb{E}\left[R_{0}\left(u_{T}^{*, l}\right)\right]}{T} \text { exists in }(-\infty, \infty)
$$

Corollary 2.8. The limit $\lim _{T \rightarrow \infty} \frac{\alpha_{T}}{T}=\frac{3}{4} B$ exists.

Proof. For all $\epsilon>0$ there exists $T_{0} \geq 1$ such that for all $T \geq T_{0}$,

$$
\limsup _{T \rightarrow \infty} \frac{\alpha_{T}}{T}=\limsup _{T \rightarrow \infty} \frac{2}{T^{2}} \int_{0}^{T / 2} \mathbb{E}\left[R_{0}\left(u_{T / 2+s}^{*, l}\right)\right] d s \leq \limsup _{T \rightarrow \infty} \frac{2}{T^{2}} \int_{0}^{T / 2}(\epsilon+B)(T / 2+s) d s=\frac{3}{4}(\epsilon+B) .
$$

Analogous reasoning for a lower bound concludes the proof.

The limit is indeed non-negative.

Lemma 2.9. The limit $B=\lim _{T \rightarrow \infty} \frac{\mathbb{E}\left[R_{0}\left(u_{T}^{*, l}\right)\right]}{T}$ from Lemma 2.7 is non-negative.

Proof. Let $\nu \in \mathcal{P}\left(\mathcal{C}_{\text {tem }}^{+}\right)$be such that $\nu\left(\left\{f: R_{0}(f)=0\right\}\right)=1$ and $\mathbb{P}_{\nu}$ is the law of a travelling wave. For $\theta>\theta_{c}$, existence follows from [19, Theorem 3.8 and (3.29)] and the shift invariance of the dynamics. By [19, Proposition 4.1], $R_{0}\left(u_{t}^{(\nu)}\right) / t$ converges a.s. to a (possibly random) limit $A^{(\nu)} \geq 0$. By monotonicity, that is by (1.13), we have $R_{0}\left(u_{t}^{*, l}\right) / t \geq R_{0}\left(u_{t}^{(\nu)}\right) / t$ for all $t \geq 1$ a.s. and thus $\liminf _{t \rightarrow \infty} R_{0}\left(u_{t}^{*, l}\right) / t \geq 0$ a.s.

Let $\epsilon>0$ arbitrary. By Corollary 2.2 there exist constants $C_{1}, C_{2}>0$ such that for $M>0$ satisfying $C_{1} e^{-C_{2} M}<\epsilon$,

$$
B \geq \limsup _{T \rightarrow \infty} \frac{\mathbb{E}\left[R_{0}\left(u_{T}^{*, l}\right) \mathbb{1}_{\left\{R_{0}\left(u_{T}^{*, l}\right) \geq-M T\right\}}\right]}{T}-\epsilon \geq \mathbb{E}\left[\liminf _{T \rightarrow \infty} \frac{R_{0}\left(u_{T}^{*, l}\right)}{T} \mathbb{1}_{\left.\left\{R_{0}\left(u_{T}^{*, l}\right)\right) \geq-M T\right\}}\right]-\epsilon \geq-\epsilon
$$

where we applied Fatou's lemma. 
We now formulate the main result of this section. The proof is deferred to Subsection 2.4.

Proposition 2.10. Let $\theta_{c}<\underline{\theta}<\bar{\theta}$. Then there exists $C=C(\underline{\theta}, \bar{\theta})>0$ and $T_{0}=T_{0}(\underline{\theta}, \bar{\theta}) \geq 1$ such that for all $T \geq T_{0}$ and $\underline{\theta} \leq \theta_{1}<\theta_{2} \leq \bar{\theta}$,

$$
\frac{\alpha_{T}\left(\theta_{2}\right)-\alpha_{T}\left(\theta_{1}\right)}{T} \geq C\left(\theta_{2}-\theta_{1}\right)
$$

Corollary 2.11. For all $\theta>\theta_{c}$,

$$
B=B(\theta)=\lim _{T \rightarrow \infty} \frac{\mathbb{E}\left[R_{0}\left(u_{T}^{*, l}(\theta)\right)\right]}{T}>0 .
$$

Proof. By definition of $\alpha_{T}$, Corollary 2.8 and Lemma 2.9, Proposition 2.10 implies that for all $\theta>\theta_{c}$,

$$
\lim _{T \rightarrow \infty} \frac{\mathbb{E}\left[R_{0}\left(u_{T}^{*, l}(\theta)\right)\right]}{T}=B(\theta)=\frac{4}{3} \lim _{T \rightarrow \infty} \frac{\alpha_{T}(\theta)}{T}>\frac{4}{3} \lim _{T \rightarrow \infty} \frac{\alpha_{T}\left(\theta_{c}+\left(\theta-\theta_{c}\right) / 2\right)}{T} \geq 0 .
$$

We conclude this subsection with two more results that we need for later estimates.

Lemma 2.12. Let $\theta_{c}<\underline{\theta}$, then there exists $\tilde{\delta}>0$ such that

$$
\mathbb{P}\left(R_{0}\left(u_{T}^{*, l}(\theta)\right) \geq 0\right) \geq \tilde{\delta}
$$

for all $T \geq 1$ and $\theta \geq \underline{\theta}$.

Proof. Use a $\theta$-*-coupling to see that it suffices to show the claim for $\underline{\theta}$ fixed. Note that for $T \geq 1$ arbitrarily fixed, $\mathbb{P}\left(R_{0}\left(u_{T}^{*, l}(\underline{\theta})\right) \geq 0\right)>0$. Therefore, in the following proof by contradiction we only need to suppose to the contrary that there exists a sequence $\left(T_{n}\right)_{n \in \mathbb{N}}$ such that $T_{n} \rightarrow \infty$ for $n \rightarrow \infty$ and $\lim _{n \rightarrow \infty} \mathbb{P}\left(R_{0}\left(u_{T_{n}}^{*, l}\right) \geq 0\right)=0$. Let $H_{0}(x)=1 \wedge(-x \vee 0)$ be Heavyside initial data and set $f_{1}(x)=$ $H_{0}(x+1)+H_{0}(-x-1)$. Then $f_{1} \in \mathcal{C}_{\text {tem }}^{+}$and $\operatorname{supp}\left(f_{1}\right)=(-\infty,-1] \cup[1, \infty)$. Let $f_{2}(x)=0 \vee(1-|x|)$, then $f_{2} \in \mathcal{C}_{c}^{+}$with $\operatorname{supp}\left(f_{2}\right)=[-1,1] . f_{1}$ fulfills condition [9, (6)] and hence [9, Theorem 1] yields $u_{t}^{\left(f_{1}\right)} \Rightarrow \mu$ for $t \rightarrow \infty$. Using a coupling with two independent processes in combination with the construction of [14, Remark 2.8(ii)], we construct two independent processes $\left(u_{t}^{*, l}\right)_{t \geq 1}$ and $\left(u_{t}^{*, r}\right)_{t \geq 1}$ such that $\mathcal{L}\left(\left(u_{t}^{*, l}\right)_{t \geq 1}\right)=$ $\mathbb{P}_{v_{1}}, \mathcal{L}\left(\left(u_{t}^{*, r}\right)_{t \geq 1}\right)=\mathbb{P}_{\kappa_{1}}$ and

$$
u_{t}^{\left(f_{1}\right)} \leq u_{t}^{*, l}(\cdot+1)+u_{t}^{*, r}(\cdot-1) \text { for all } t \geq 1, x \in \mathbb{R} \text { almost surely. }
$$

By [9, Theorem 1] and [16, Theorem 1],

$$
\int_{\mathcal{C}_{\text {tem }}^{+}} e^{-2\left\langle g, f_{2}\right\rangle} \mu(d g)=\mathbb{P}_{f_{2}}(\tau<\infty)<1 .
$$

Note that the additional factor of 2 in the exponent results from the use of a different scaling constant in the original SPDE. We obtain by the weak convergence of $u_{t}^{\left(f_{1}\right)}$ to $\mu$,

$$
\begin{aligned}
1 & >\lim _{n \rightarrow \infty} \int_{\mathcal{C}_{\text {tem }}^{+}} e^{-2\left\langle g, f_{2}\right\rangle} u_{T_{n}}^{\left(f_{1}\right)}(d g)=\lim _{n \rightarrow \infty} \mathbb{E}\left[e^{-2\left\langle u_{T_{n}}^{\left(f_{1}\right)}, f_{2}\right\rangle}\right] \\
& \geq \lim _{n \rightarrow \infty} \mathbb{E}\left[e^{-2\left\langle u_{T_{n}}^{*, l}(\cdot+1)+u_{T_{n}}^{*, r}(\cdot-1), f_{2}\right\rangle}\right]=\lim _{n \rightarrow \infty} \mathbb{E}\left[e^{-2\left\langle u_{T_{n}}^{*, l}(\cdot+1), f_{2}\right\rangle}\right] \mathbb{E}\left[e^{-2\left\langle u_{T_{n}}^{*, r}(\cdot-1), f_{2}\right\rangle}\right] .
\end{aligned}
$$

The assumption $\lim _{n \rightarrow \infty} \mathbb{P}\left(R_{0}\left(u_{T_{n}}^{*, l}\right) \geq 0\right)=0$ yields by symmetry and by the shift invariance of the dynamics, $\lim _{n \rightarrow \infty} \mathbb{P}\left(R_{0}\left(u_{T_{n}}^{*, l}(\cdot+1) \geq-1\right)=0=\lim _{n \rightarrow \infty} \mathbb{P}\left(L_{0}\left(u_{T_{n}}^{*, r}(\cdot-1) \leq 1\right)=0\right.\right.$. Use a coupling $u_{T_{n}}^{*, r} \leq u_{T_{n}}^{*}$ with $u_{T_{n}}^{*} \Rightarrow \mu \in \mathcal{P}\left(\mathcal{C}_{\text {tem }}^{+}\right)$(cf. [14, (2.34) and Proposition 2.4]) to conclude by using dominated convergence that the right hand side in (2.23) is equal to 1 , a contradiction. 
Lemma 2.13. For all $\theta_{c}<\theta \leq \bar{\theta}, T \geq 1$,

$$
\mathbb{E}\left[\left(0 \vee R_{0}\left(u_{T}^{*, l}\right)\right)^{2}\right] \leq C(\bar{\theta}) T^{2}
$$

Proof. In what follows, constants $C=C(\bar{\theta})$ may change from line to line. Note that for $a_{i} \geq 0, i=$ $1, \ldots, n, n \in \mathbb{N},\left(\sum_{i=1}^{n} a_{i}\right)^{2} \leq n \sum_{i=1}^{n} a_{i}^{2}$.

We first show the claim for $T \in \mathbb{N}$. Reason as in [14, Lemma 4.2-Proposition 4.5] to show that for $T=1, \mathbb{E}\left[\left(0 \vee R_{0}\left(u_{1}^{*, l}\right)\right)^{2}\right] \leq C$. Then reason as in [14, Lemma 4.6] to show the claim for $T \in \mathbb{N}$ by induction.

Next, we extend this result to $T \geq 1$. As $\mathcal{L}\left(u_{T}^{*, l}\right) \in \mathcal{P}\left(\mathcal{C}_{\text {tem }}^{+} \backslash\{0\}\right)$ for all $T>0$ we use [14, Remark 2.8] to get for $T \geq 1$ arbitrary,

$$
\mathbb{E}\left[\left(0 \vee R_{0}\left(u_{T}^{*, l}\right)\right)^{2}\right]=\mathbb{E}\left[\left(0 \vee R_{0}\left(u_{T-\lfloor T\rfloor}^{\left(u_{\lfloor T\rfloor}^{*, l}\right)}\right)\right)^{2}\right]
$$

By [14, Remark A.1] and symmetry,

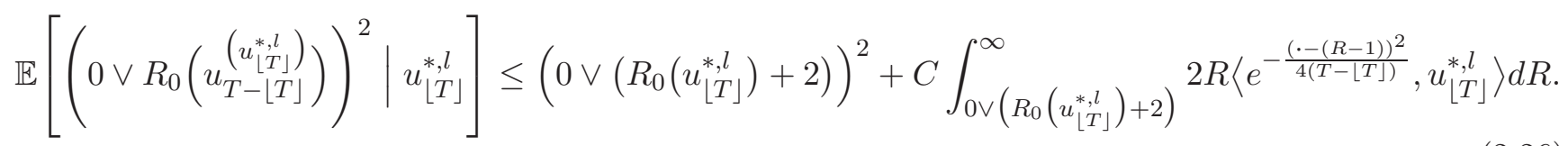

Take expectations and use [14, Corollaries 2.6 and 2.9] to conclude that

$$
\mathbb{E}\left[\left(0 \vee R_{0}\left(u_{T-\lfloor T\rfloor}^{\left(u_{\lfloor T\rfloor}^{*, l}\right)}\right)\right)^{2}\right] \leq C\lfloor T\rfloor^{2}+C \int_{0}^{\infty} 2 R\left\langle e^{-\frac{(\cdot-(R-1))^{2}}{4}}, 1\right\rangle d R \leq C T^{2}
$$

as claimed.

\subsection{A preliminary estimate}

The following two lemmas yield, in combination, a lower bound on the expected increase of the right front marker at time $T+t, T>0, t \geq 0$ resulting from an increase of $\psi \in \mathcal{C}_{\text {tem }}^{+}$in the initial density of a solution to (1.1).

Recall the construction of the left upper invariant measure $v_{T}$ and the process $\left(u_{T+t}^{*, l}\right)_{t>0}$ for $T>0$ fixed from [14] (cf. the corresponding construction for the upper invariant measure $\mu_{T}$ from [14], Proposition 2.2 and Corollary 2.6 as well as Remark 2.8). For arbitrarily fixed (to be chosen later) $\psi \in \mathcal{C}_{\text {tem }}^{+}$, write

$$
\Phi(x) \equiv\left\{\begin{array} { l l } 
{ \infty , } & { x < 0 , } \\
{ 0 , } & { \text { otherwise } }
\end{array} \quad \text { and } \quad \Psi ( x ) \equiv \left\{\begin{array}{ll}
\infty, & x<0 \\
\psi(x), & x \geq 0 \\
0, & \text { otherwise }
\end{array}\right.\right.
$$

In what follows consider couplings of solutions $\left(u_{T+t}^{(\phi)}\right)_{t \geq 0}, \phi \in \mathcal{C}_{\text {tem }}^{+}$and $\left(u_{T+t}^{(\phi+\psi)}\right)_{t \geq 0}, \psi \in \mathcal{C}_{t e m}^{+}$with processes $\left(u_{T+t}^{(\Phi)}\right)_{t \geq 0}$ and $\left(u_{T+t}^{(\Psi)}\right)_{t \geq 0}$ for $T>0$ arbitrarily fixed. Note that by a slight abuse of notation $" \Psi=\Phi+\psi$ ". The two latter processes are to be understood in the spirit of the construction of $v_{T}$, that is as in Corollary 2.6 we choose sequences $\left(\Psi_{N}\right)_{N \in \mathbb{N}}$ and $\left(\Phi_{N}\right)_{N \in \mathbb{N}}$ such that $\Psi_{N} \uparrow \Psi$ and $\Phi_{N} \uparrow \Phi$ for $N \rightarrow \infty$ to obtain $u_{T+t}^{(\Psi)}(x) \equiv \uparrow \lim _{N \rightarrow \infty} u_{T+t}^{\left(\Psi_{N}\right)}(x)$ and $u_{T+t}^{(\Phi)} \equiv \uparrow \lim _{N \rightarrow \infty} u_{T+t}^{\left(\Phi_{N}\right)}(x)$ on a common probability space. 
Lemma 2.14. Let $\psi \in \mathcal{C}_{\text {tem }}^{+}$arbitrarily fixed and $\Phi, \Psi$ be as above. Let $\phi \in \mathcal{C}_{\text {tem }}^{+}$arbitrary with $R_{0}(\phi) \leq 0$. Then, for arbitrary $T>0, t \geq 0$, there exists a coupling of processes $\left(u_{T+t}^{(\Phi)}\right)_{t \geq 0},\left(u_{T+t}^{(\Psi)}\right)_{t \geq 0}$ and solutions $\left(u_{T+t}^{(\phi)}\right)_{t \geq 0},\left(u_{T+t}^{(\phi+\psi)}\right)_{t \geq 0}$ such that

$$
\mathbb{E}\left[R_{0}\left(u_{T+t}^{(\Psi)}\right)-R_{0}\left(u_{T+t}^{(\Phi)}\right)\right] \leq \mathbb{E}\left[R_{0}\left(u_{T+t}^{(\phi+\psi)}\right)-R_{0}\left(u_{T+t}^{(\phi)}\right)\right]
$$

for all $t \geq 0$ almost surely. On the right hand side we consider a monotonicity-coupling and set $R_{0}\left(u_{T+t}^{(\phi+\psi)}\right)-R_{0}\left(u_{T+t}^{(\phi)}\right)=0$ on $\left\{\tau^{(\phi+\psi)} \leq T+t\right\}$.

Remark 2.15. Note that the expectations on the left hand side of (2.29) are well-defined by Lemma 2.1] and Corollaries 2.3 2.4. Indeed, note that if $f_{n} \uparrow f$ in $\mathcal{C}_{\text {tem }}^{+}$, then $R_{0}\left(f_{n}\right) \uparrow R_{0}(f)$ for $n \rightarrow \infty$. Now use approximating sequences $\Psi_{N}, \Phi_{N} \in \mathcal{H}^{R}$ for $\Psi$ respectively $\Phi$ from below as in [14, Remark 2.8(i)] in combination with dominated convergence.

Proof. Step 1. Let $\phi, \psi$ as in the statement above. We first show the claim for $T=0$ and $\Phi, \Psi \in \mathcal{C}_{\text {tem }}^{+}$ satisfying $R_{0}(\Phi)=0, \Phi \geq \phi$ and $\Psi=\Phi+\psi$. Consider the following coupling. Let $u_{1}=u^{(\phi)}$ be a non-negative solution to

$$
\frac{\partial u_{1}}{\partial t}=\Delta u_{1}+\left(\theta-u_{1}\right) u_{1}+\sqrt{u_{1}} \dot{W}_{1}, \quad u_{1}(0)=\phi
$$

and $v_{2}$ be a non-negative solution to

$$
\frac{\partial v_{2}}{\partial t}=\Delta v_{2}+\left(\theta-v_{2}-2 u_{1}\right) v_{2}+\sqrt{v_{2}} \dot{W}_{2}, \quad v_{2}(0)=\Phi-\phi
$$

with $W_{2}$ a white noise independent of $W_{1}$. For the construction of the latter proceed as in Remark 5.9 on monotonicity-couplings. Then $u_{1}+v_{2} \stackrel{\mathcal{D}}{=} u^{(\Phi)}$, that is, $u_{1}+v_{2}$ solves (1.1) with initial condition $\Phi$. Let $v_{3}$ be a non-negative solution to

$$
\frac{\partial v_{3}}{\partial t}=\Delta v_{3}+\left(\theta-v_{3}-2\left(u_{1}+v_{2}\right)\right) v_{3}+\sqrt{v_{3}} \dot{W}_{3}, \quad v_{3}(0)=\psi
$$

with $W_{3}$ a white noise independent of $W_{1}, W_{2}$. Then $u_{1}+v_{2}+v_{3} \stackrel{\mathcal{D}}{=} u^{(\Phi+\psi)}$ follows as above, and using that $\Psi=\Phi+\psi$,

$$
\begin{aligned}
R_{0}\left(u_{t}^{(\Psi)}\right)-R_{0}\left(u_{t}^{(\Phi)}\right) & \stackrel{\mathcal{D}}{=} R_{0}\left(\left(u_{1}+v_{2}+v_{3}\right)_{t}\right)-R_{0}\left(\left(u_{1}+v_{2}\right)_{t}\right) \\
& =\left(R_{0}\left(\left(v_{3}\right)_{t}\right)-R_{0}\left(\left(u_{1}+v_{2}\right)_{t}\right)\right) \vee 0
\end{aligned}
$$

for all $t \geq 0$ a.s., where we set $R_{0}\left(u_{t}^{(\Psi)}\right)-R_{0}\left(u_{t}^{(\Phi)}\right)=0$ on $\left\{\tau^{(\Psi)} \leq t\right\}$. Finally, let $d_{4}$ be a non-negative solution to

$$
\frac{\partial d_{4}}{\partial t}=\Delta d_{4}+2 v_{2} v_{3}+\left(\theta-d_{4}-2\left(u_{1}+v_{3}\right)\right) d_{4}+\sqrt{d_{4}} \dot{W}_{4}, \quad d_{4}(0)=0
$$

with $W_{4}$ independent of $W_{1}, W_{2}, W_{3}$ and where the term $2 v_{2} v_{3}$ can be interpreted as an additional immigration term. Then $u_{1}+v_{3}+d_{4} \stackrel{\mathcal{D}}{=} u^{(\phi+\psi)}$ and

$$
\begin{aligned}
R_{0}\left(u_{t}^{(\phi+\psi)}\right)-R_{0}\left(u_{t}^{(\phi)}\right) & =R_{0}\left(\left(u_{1}+v_{3}+d_{4}\right)_{t}\right)-R_{0}\left(\left(u_{1}\right)_{t}\right) \\
& =\left(R_{0}\left(\left(v_{3}+d_{4}\right)_{t}\right)-R_{0}\left(\left(u_{1}\right)_{t}\right)\right) \vee 0 \\
& \geq\left(R_{0}\left(\left(v_{3}\right)_{t}\right)-R_{0}\left(\left(u_{1}+v_{2}\right)_{t}\right)\right) \vee 0,
\end{aligned}
$$

the last by the non-negativity of the solutions $d_{4}$ and $v_{2}$. 
The second part of the claim now follows from the above and (2.33). For the first part of the claim, use that $\Psi=\Phi+\psi$ and

$$
u_{1} \stackrel{\mathcal{D}}{=} u^{(\phi)}, \quad u_{1}+v_{2} \stackrel{\mathcal{D}}{=} u^{(\Phi)}, \quad u_{1}+v_{2}+v_{3} \stackrel{\mathcal{D}}{=} u^{(\Phi+\psi)}, \quad u_{1}+v_{3}+d_{4} \stackrel{\mathcal{D}}{=} u^{(\phi+\psi)}
$$

to obtain a coupling satisfying

$$
u_{t}^{(\Psi)}-u_{t}^{(\Phi)}=\left(u_{1}+v_{2}+v_{3}\right)-\left(u_{1}+v_{2}\right)=v_{3} \leq v_{3}+d_{4}=\left(u_{1}+v_{3}+d_{4}\right)-u_{1}=u_{t}^{(\phi+\psi)}-u_{t}^{(\phi)}
$$

as claimed.

Step 2. Fix $T>0$. Let $\Phi_{N} \uparrow \Phi, \Phi$ as in (2.28), satisfy $R_{0}\left(\Phi_{N}\right)=0$ and $\Phi_{1} \geq \phi, \Phi_{1} \in \mathcal{H}^{R}$. Set $\Psi_{N}=\Phi_{N}+\psi$. By Step 1, there exists a coupling of solutions $\left(u_{T+t}^{\left(\Phi_{N}\right)}\right)_{t \geq 0},\left(u_{T+t}^{\left(\Psi_{N}\right)}\right)_{t \geq 0}$ to (1.1) such that (2.29) holds with $\Phi, \Psi$ replaced by $\Phi_{N}, \Psi_{N}$ for $N \in \mathbb{N}$ arbitrarily fixed.

Define $u_{T+t}^{(\Phi)}(x)=\uparrow \lim _{N \rightarrow \infty} u_{T+t}^{\left(\Phi_{N}\right)}(x)$ and $u_{T+t}^{(\Psi)}(x)=\uparrow \lim _{N \rightarrow \infty} u_{T+t}^{\left(\Psi_{N}\right)}(x)$ on a common probability space (cf. [14, Remark 2.8(i)]). By taking limits in $N \rightarrow \infty$, the claim now follows for $\Phi, \Psi$ as well by dominated convergence (cf. Remark 2.15 above).

Lemma 2.16. For $t>0$ fixed and $\psi \in \mathcal{C}_{\text {tem }}^{+}$,

$$
\mathbb{E}\left[R_{0}\left(u_{t}^{(\Psi)}\right) \vee 0-R_{0}\left(u_{t}^{(\Phi)}\right) \vee 0\right] \geq \int_{0}^{\infty} \mathbb{E}\left[\mathbb{1}_{\left\{-x \leq L_{0}\left(u_{t}^{*, r}\right)<-x+1\right\}}\left(1-e^{-2\left\langle\mathbb{1}_{[0,1)} \psi, u_{t}^{*, r}(\cdot-x)\right\rangle}\right)\right] d x
$$

holds.

Proof. By partial integration, for $\phi \in \mathcal{C}_{\text {tem }}^{+}, t>0$ arbitrary,

$$
\mathbb{E}_{\phi}\left[R_{0}\left(u_{t}\right) \vee 0\right]=\int_{0}^{\infty} \mathbb{P}_{\phi}\left(R_{0}\left(u_{t}\right)>x\right) d x
$$

By (1.11), symmetry and by the shift invariance of the dynamics,

$$
\mathbb{P}_{\phi}\left(R_{0}\left(u_{t}\right) \leq x\right)=\mathbb{E}\left[e^{-2\left\langle\phi, u_{t}^{*, r}(\cdot-x)\right\rangle}\right]=\mathbb{E}\left[e^{-2\left\langle\phi(\cdot+x), u_{t}^{*, r}\right\rangle}\right] .
$$

Hence,

$\mathbb{E}_{\phi}\left[R_{0}\left(u_{t}\right) \vee 0\right]=\int_{0}^{\infty} \mathbb{E}\left[\left(1-e^{-2\left\langle\phi, u_{t}^{*, r}(\cdot-x)\right\rangle}\right)\right] d x=\int_{0}^{\infty} \mathbb{E}\left[\mathbb{1}_{\left\{u_{t}^{*, r} \mid \operatorname{supp}(\phi(\cdot+x)) \not \equiv 0\right\}}\left(1-e^{-2\left\langle\phi, u_{t}^{*, r}(\cdot-x)\right\rangle}\right)\right] d x$

In the following we use $\Phi$ and $\Psi=\Phi+\psi$ as initial conditions or test functions to facilitate notation. This notation is understood as an abbreviation for taking limits of non-decreasing approximating sequences of initial conditions as explained above and using monotone convergence to obtain the respective results.

Reason as in Remark 2.15 to see that the following integrals are well-defined. The Theorem of FubiniTonelli yields

$$
\begin{aligned}
\mathbb{E}\left[R_{0}\left(u_{t}^{(\Psi)}\right) \vee 0-R_{0}\left(u_{t}^{(\Phi)}\right) \vee 0\right] & =\int_{0}^{\infty} \mathbb{E}\left[e^{-2\left\langle\Phi(\cdot+x), u_{t}^{*, r}\right\rangle}\right]-\mathbb{E}\left[e^{-2\left\langle\Psi(\cdot+x), u_{t}^{*, r}\right\rangle}\right] d x \\
& =\int_{0}^{\infty} \mathbb{E}\left[e^{-2\left\langle\Phi(\cdot+x), u_{t}^{*, r}\right\rangle}\left(1-e^{-2\left\langle\psi(\cdot+x), u_{t}^{*, r}\right\rangle}\right)\right] d x \\
& =\int_{0}^{\infty} \mathbb{E}\left[\mathbb{1}_{\left\{L_{0}\left(u_{t}^{*, r}\right) \geq-x\right\}}\left(1-e^{-2\left\langle\psi(\cdot+x), u_{t}^{*, r}\right\rangle}\right)\right] d x \\
& \geq \int_{0}^{\infty} \mathbb{E}\left[\mathbb{1}_{\left\{-x \leq L_{0}\left(u_{t}^{*, r}\right)<-x+1\right\}}\left(1-e^{-2\left\langle\mathbb{1}_{[0,1)} \psi, u_{t}^{*, r}(\cdot-x)\right\rangle}\right)\right] d x
\end{aligned}
$$

This completes the proof. 


\subsection{Proof of Proposition 2.10}

Let $T \geq 1$ be arbitrarily fixed. For $\theta_{c}<\underline{\theta}<\bar{\theta}$ arbitrary let

$$
\delta=\frac{\bar{\theta}-\underline{\theta}}{M} \text {, where } M>0 \text { is arbitrarily large with } M T \in \mathbb{N} \text { and } \theta_{m}=\underline{\theta}+m \frac{\delta}{T}, m \in\{0,1, \ldots, M T\} .
$$

For ease of notation, we only prove the case $\theta_{1}=\underline{\theta}, \theta_{2}=\bar{\theta}$. Note that if we let $\delta=\left(\theta_{2}-\theta_{1}\right) / M$ instead and consider the difference $\alpha_{T}\left(\theta_{2}\right)-\alpha_{T}\left(\theta_{1}\right)$ in what follows, the proof remains unchanged.

We proceed to observe that $\theta_{0}=\underline{\theta}, \theta_{M T}=\bar{\theta}$ and that we therefore rewrite

$$
\begin{aligned}
\alpha_{T}(\bar{\theta})-\alpha_{T}(\underline{\theta}) & =\sum_{m=1}^{M T}\left\{\alpha_{T}\left(\theta_{m}\right)-\alpha_{T}\left(\theta_{m-1}\right)\right\} \\
& =\sum_{m=1}^{M T} \frac{2}{T} \int_{0}^{T / 2}\left\{\mathbb{E}\left[R_{0}\left(u_{T / 2+s}^{*, l}\left(\theta_{m}\right)\right)\right]-\mathbb{E}\left[R_{0}\left(u_{T / 2+s}^{*, l}\left(\theta_{m-1}\right)\right]\right\} d s .\right.
\end{aligned}
$$

Let $\xi>0$ arbitrary and $S=S(\omega, m), m \in \mathbb{N}$ with $\xi \leq S \leq T / 2-\xi$ be random stopping times to be made more precise later on. Then, by the strong Markov property of the processes involved,

$$
\alpha_{T}(\bar{\theta})-\alpha_{T}(\underline{\theta})=\sum_{m=1}^{M T} \frac{2}{T} \int_{0}^{T / 2} \mathbb{E}\left[\mathbb{E}_{u_{S}^{*, l}\left(\theta_{m}\right)}\left[R_{0}\left(u_{T / 2-S+s}\left(\theta_{m}\right)\right)\right]-\mathbb{E}_{u_{S}^{*, l}\left(\theta_{m-1}\right)}\left[R_{0}\left(u_{T / 2-S+s}\left(\theta_{m-1}\right)\right)\right]\right] d s .
$$

The expectations are well-defined by Corollaries 2.3 2.4. Using a $\theta$-coupling we bound (2.45) from below by

$$
\sum_{m=1}^{M T} \frac{2}{T} \int_{0}^{T / 2} \mathbb{E}\left[\mathbb{E}_{u_{S}^{*,}\left(\theta_{m}\right)}\left[R_{0}\left(u_{T / 2-S+s}\left(\theta_{m-1}\right)\right)\right]-\mathbb{E}_{u_{S}^{*, l}\left(\theta_{m-1}\right)}\left[R_{0}\left(u_{T / 2-S+s}\left(\theta_{m-1}\right)\right)\right]\right] d s .
$$

A shift in space, using the shift invariance of the dynamics, further allows to rewrite this to

$$
\begin{aligned}
\sum_{m=1}^{M T} \frac{2}{T} & \int_{0}^{T / 2} \mathbb{E}\left[\mathbb{E}_{u_{S}^{*, l}\left(\theta_{m}\right)\left(\cdot+R_{0}\left(u_{S}^{*, l}\left(\theta_{m-1}\right)\right)\right)}\left[R_{0}\left(u_{T / 2-S+s}\left(\theta_{m-1}\right)\right)\right]\right. \\
& -\mathbb{E}_{u_{S}^{*, l}\left(\theta_{m-1}\right)}\left(\cdot+R_{0}\left(u_{S}^{*, l}\left(\theta_{m-1}\right)\right)\right) \\
& {\left.\left[R_{0}\left(u_{T / 2-S+s}\left(\theta_{m-1}\right)\right)\right]\right] d s . }
\end{aligned}
$$

For $S \geq \xi>0$, use a $\theta$-*-coupling to obtain

$$
0 \leq \Delta_{S}^{*, l}\left(\theta_{m-1}, \theta_{m}\right) \equiv u_{S}^{*, l}\left(\theta_{m}\right)\left(\cdot+R_{0}\left(u_{S}^{*, l}\left(\theta_{m-1}\right)\right)\right)-u_{S}^{*, l}\left(\theta_{m-1}\right)\left(\cdot+R_{0}\left(u_{S}^{*, l}\left(\theta_{m-1}\right)\right)\right) \in \mathcal{C}_{t e m}^{+} .
$$

Hence, we use the strong Markov property of the family of laws $\mathbb{P}_{f}, f \in \mathcal{C}_{\text {tem }}^{+}$to apply Lemma 2.14, using that $T / 2-S \geq \xi>0$ and $S \geq \xi>0$, to see that

$$
\begin{aligned}
& \alpha_{T}(\bar{\theta})-\alpha_{T}(\underline{\theta}) \\
& \geq \sum_{m=1}^{M T} \frac{2}{T} \int_{0}^{T / 2} \mathbb{E}\left[\mathbb { E } \left[\mathbb{E}_{u_{S}^{*, l}\left(\theta_{m}\right)\left(\cdot+R_{0}\left(u_{S}^{*, l}\left(\theta_{m-1}\right)\right)\right)}\left[R_{0}\left(u_{T / 2-S+s}\left(\theta_{m-1}\right)\right)\right]\right.\right. \\
& \left.\left.-\mathbb{E}_{u_{S}^{*, l}\left(\theta_{m-1}\right)\left(\cdot+R_{0}\left(u_{S}^{*, l}\left(\theta_{m-1}\right)\right)\right)}\left[R_{0}\left(u_{T / 2-S+s}\left(\theta_{m-1}\right)\right)\right] \mid \mathcal{F}_{S}\right]\right] d s \\
& \left.\left.\geq \sum_{m=1}^{M T} \frac{2}{T} \int_{0}^{T / 2} \mathbb{E}\left[\mathbb{E}\left[R_{0}\left(u_{T / 2-S+s}^{\left(\Phi+\Delta_{S}^{*, l}\left(\theta_{m-1}, \theta_{m}\right.\right.}\right)\right)\left(\theta_{m-1}\right)\right)\right]-\mathbb{E}\left[R_{0}\left(u_{T / 2-S+s}^{(\Phi)}\left(\theta_{m-1}\right)\right)\right]\right] d s \\
& \geq \sum_{m=1}^{M T} \frac{2}{T} \int_{0}^{T / 2} \mathbb{E}\left[R_{0}\left(u_{T / 2-S+s}^{\left(\Phi+\Delta_{S}^{*, l}\left(\theta_{m-1}, \theta_{m}\right)\right)}\left(\theta_{m-1}\right)\right) \vee 0-R_{0}\left(u_{T / 2-S+s}^{(\Phi)}\left(\theta_{m-1}\right)\right) \vee 0\right] d s \text {. }
\end{aligned}
$$


With the help of Lemma 2.16 we further bound this from below by

$$
\sum_{m=1}^{M T} \frac{2}{T} \int_{0}^{T / 2} \int_{0}^{\infty} \mathbb{E}\left[\mathbb{1}_{\left\{-x \leq L_{0}\left(u_{T / 2-S+s}^{*, r}\left(\theta_{m-1}\right)\right)<-x+1\right\}}\left(1-e^{-2\left\langle\mathbb{1}_{[0,1)} \Delta_{S}^{*, l}\left(\theta_{m-1}, \theta_{m}\right),\left(u_{T / 2-S+s}^{*, r}\left(\theta_{m-1}\right)\right)(\cdot-x)\right\rangle}\right)\right] d x d s .
$$

For $d_{0}, m_{0}>0$, let

$$
\tilde{M}\left(d_{0}, m_{0}\right)=\left\{f \in \mathcal{C}_{\text {tem }}^{+}: \text {there exist } 0 \leq l_{0}<r_{0} \leq 1 / 2 \text { with }\left|r_{0}-l_{0}\right|=d_{0} \text { such that } f \geq m_{0} \mathbb{1}_{\left[l_{0}, r_{0}\right]}\right\} \text {. }
$$

Fix $\epsilon>0$ arbitrary and let $d_{0}=d_{0}(\epsilon), m_{0}=m_{0}(\epsilon)>0$ as in Corollary [5.5, where we note that instead of considering right markers we now consider left markers. We obtain as a further lower bound to the above

$$
\begin{aligned}
& \sum_{m=1}^{M T} \frac{2}{T} \int_{0}^{T / 2} \int_{0}^{\infty} \mathbb{E}\left[\mathbb{1}_{\left\{-x \leq L_{0}\left(u_{T / 2-S+s}^{*, r}\left(\theta_{m-1}\right)\right)<-x+1\right\}^{1}} \mathbb{1}_{\left\{u_{T / 2-S+s}^{*, r}\left(\theta_{m-1}\right)\left(\cdot+L_{0}\left(u_{T / 2-S+s}^{*, r}\left(\theta_{m-1}\right)\right)\right) \in \tilde{M}\left(d_{0}, m_{0}\right)\right\}}\right. \\
& \quad \times\left(1-e^{\left.\left.-2\left\langle\mathbb{1}_{[0,1)} \Delta_{S}^{*, l}\left(\theta_{m-1}, \theta_{m}\right),\left(u_{T / 2-S+s}^{*, r}\left(\theta_{m-1}\right)\right)(\cdot-x)\right\rangle\right)\right] d x d s}\right.
\end{aligned}
$$

for all $T \geq 1$. We next make use of the following crucial observation. Recall that $\theta_{m}-\theta_{m-1}=\delta / T$ for $m \in\{1, \ldots, M T\}$ with $\delta=(\bar{\theta}-\underline{\theta}) / M$.

Proposition 2.17. For all $\xi>0$ and $\varphi \in \mathcal{C}_{\text {tem }}^{+}$with $L_{0}(\varphi) \in(0,1)$ there exist $T_{0}>0$ big enough and $\rho, C_{0}, C_{1}>0$ small enough, all constants only dependent on $\xi, \underline{\theta}, \bar{\theta}, \varphi$, such that for all $T \geq T_{0}$ and $m \in\{0,1, \ldots, M T\}, M \in \mathbb{N}$ there exist stopping times $\xi \leq S=S(m, \varphi) \leq T / 2-\xi$ such that

$$
\mathbb{P}\left(\left\langle\Delta_{S(m, \varphi)}^{*, l}\left(\theta_{m-1}, \theta_{m}\right), \varphi\right\rangle \geq \rho\right) \geq C_{0}\left(1-\exp \left(-C_{1} \delta\right)\right) .
$$

The proof of the proposition follows in Section 3 below. First, we finish the proof of Proposition 2.10 , We obtain as a lower bound to the term in (2.52) with $\varphi=m_{0} \mathbb{1}_{\left[1 / 2,1 / 2+d_{0} / 2\right]}$,

$$
\begin{aligned}
& C_{0}\left(1-e^{-C_{1} \delta}\right)\left(1-e^{-2 \rho}\right) \sum_{m=1}^{M T} \frac{2}{T} \int_{0}^{T / 2} \int_{0}^{\infty} \mathbb{E}\left[\mathbb{1}_{\left\{-x \leq L_{0}\left(u_{T / 2-S+s}^{*, r}\left(\theta_{m-1}\right)\right)<-x+1\right\}}\right.
\end{aligned}
$$

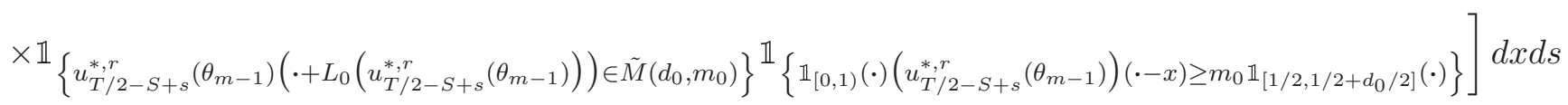

for all $T \geq T_{0}$. By definition of $\tilde{M}\left(d_{0}, m_{0}\right)$, using the Theorem of Fubini-Tonelli, this is bounded from below by

$$
\begin{aligned}
& C_{0}\left(1-e^{-C_{1} \delta}\right)\left(1-e^{-2 \rho}\right) \frac{d_{0}}{2} \sum_{m=1}^{M T} \frac{2}{T} \int_{0}^{T / 2} \mathbb{E}\left[\mathbb{1}_{\left\{L_{0}\left(u_{T / 2-S+s}^{*, r}\left(\theta_{m-1}\right)\right)<0\right\}}\right. \\
& \quad \times \mathbb{1}_{\left.\left\{u_{T / 2-S+s}^{*, r}\left(\theta_{m-1}\right)\left(\cdot+L_{0}\left(u_{T / 2-S+s}^{*, r}\left(\theta_{m-1}\right)\right)\right) \in \tilde{M}\left(d_{0}, m_{0}\right)\right\}\right] d s .}
\end{aligned}
$$

By symmetry and Lemma 2.12, we have for $T$ big enough,

$$
\frac{2}{T} \int_{0}^{T / 2} \mathbb{E}\left[\mathbb{1}_{\left\{L_{0}\left(u_{T / 2-S+s}^{*, r}\left(\theta_{m-1}\right)\right)<0\right\}}\right] d s \geq \tilde{\delta} / 2>0
$$


with $\tilde{\delta}$ as in Lemma 2.12, Recall the definition of $\left(\nu_{T}^{*, l}(\theta)\right)$ from (5.8). We conclude using Corollary 5.5 and symmetry that

$$
\begin{aligned}
\alpha_{T}(\bar{\theta})-\alpha_{T}(\underline{\theta}) & \geq C_{0}\left(1-e^{-C_{1} \delta}\right)\left(1-e^{-2 \rho}\right) \frac{d_{0}}{2} \sum_{m=1}^{M T}(\tilde{\delta} / 2-\epsilon) \\
& =C_{0}\left(1-e^{-C_{1} \delta}\right)\left(1-e^{-2 \rho}\right) \frac{d_{0}}{2}(\tilde{\delta} / 2-\epsilon) M T
\end{aligned}
$$

for all $T \geq T_{0}$. Choose $\epsilon$ small enough and recall that $\delta=(\bar{\theta}-\underline{\theta}) / M$ respectively $M=(\bar{\theta}-\underline{\theta}) / \delta$ to conclude that

$$
\frac{\alpha_{T}(\bar{\theta})-\alpha_{T}(\underline{\theta})}{T} \geq C_{0}\left(1-e^{-2 \rho}\right) \frac{d_{0}}{2}(\tilde{\delta} / 2-\epsilon)\left(1-e^{-C_{1}(\bar{\theta}-\underline{\theta}) / M}\right) M .
$$

Let $M \rightarrow \infty$ to obtain $C_{0}\left(1-e^{-2 \rho}\right) \frac{d_{0}}{2}(\tilde{\delta} / 2-\epsilon)(\bar{\theta}-\underline{\theta})$ as a lower bound to the left hand side. This finishes the proof.

\subsection{Proof of Proposition 1.1}

Lemma 2.7yields the existence of the limit $B=B(\theta)$. Its positivity follows from Corollary 2.11. Combine Proposition 2.10 and Corollary 2.8 to obtain (1.14) by taking $T \rightarrow \infty$. This concludes the proof.

\section{Proof of Proposition 2.17}

In this section we prove Proposition 2.17 We start out by giving the main idea of the proof.

\subsection{Idea of proof}

Let $T_{1}, T_{2}>0, m \in \mathbb{N}$ and $\xi>0$ be arbitrarily fixed. Let $\theta_{m}$ as in (2.43) and suppose that $\theta_{c}<\underline{\theta} \leq$ $\theta_{m-1}<\theta_{m} \leq \bar{\theta}$. For $t \in\left[\xi, T / 2-\xi-T_{1}-T_{2}\right]$ fixed, on a time-interval of length $T_{1}+T_{2}$, we look for a (random) point in time $S=S(m) \in\left[t, t+T_{1}+T_{2}\right]$ such that

$$
\mathbb{1}_{[0,1)} \Delta_{S}^{*, l}\left(\theta_{m-1}, \theta_{m}\right) \geq \rho \mathbb{1}_{[0,1)}
$$

where

$$
\Delta_{S}^{*, l}\left(\theta_{m-1}, \theta_{m}\right) \equiv u_{S}^{*, l}\left(\theta_{m}\right)\left(\cdot+R_{0}\left(u_{S}^{*, l}\left(\theta_{m-1}\right)\right)\right)-u_{S}^{*, l}\left(\theta_{m-1}\right)\left(\cdot+R_{0}\left(u_{S}^{*, l}\left(\theta_{m-1}\right)\right)\right)
$$

as in (2.48).

We investigate the difference between the solutions $u^{*, l}\left(\theta_{m}\right)$ and $u^{*, l}\left(\theta_{m-1}\right)$ over time with the goal of finding $S$ such that (3.1) holds. For $t$ fixed as above, condition on $\mathcal{F}_{t}$. Aside from the shift in space, by monotonicity, the difference on the time-interval $\left[t, t+T_{1}+T_{2}\right]$ is greater or equal to the difference of solutions $u_{t+.}^{*, l}\left(\theta_{m}\right)$ and $u_{t+.}^{*, l}\left(\theta_{m-1}\right)$ with common initial condition $u_{t}^{*, l}\left(\theta_{m-1}\right)$ at time $t$.

First step: Start out with density $u_{t}^{*, l}\left(\theta_{m-1}\right)$. Use a time-interval of length $T_{1}$ to gain additional massdensity $v_{T_{1}}$ of height of order $O(\epsilon), \epsilon \equiv \theta_{m}-\theta_{m-1}$ on the support of $u_{t}^{*, l}\left(\theta_{m-1}\right)$ with probability of order $O(1)$. This amount is due to an immigration term of order $\epsilon u_{t+s}^{*, l}\left(\theta_{m-1}\right), s \in\left[0, T_{1}\right]$ in a $\theta$-coupling. For $T_{1}$ not too big, the mass created, $v_{s}, s \in\left[0, T_{1}\right]$ remains small and immigration dominates the annihilation term of order $v_{s}$.

Second step: Use a monotonicity-coupling to compare the original solution $u_{t+T_{1}+\cdot}^{*, l}\left(\theta_{m-1}\right)$ with parameter $\theta_{m-1}$ for a time-interval of length $T_{2}$ with a solution with the same parameter $\theta_{m-1}$ but with mass $v_{T_{1}}$ (cf. first step) added to the initial condition (at time $t+T_{1}$ ). With probability of order $O(\epsilon)$ the mass

$v_{T_{1}}$ gets a constant distance and an amount of mass $O(1)$ in front of the original solution $u_{t+T_{1}+\cdot}^{*, l}\left(\theta_{m-1}\right)$ after a time-period of length $T_{2}$. To be more precise, we use this time-period of length $T_{2}$ twofold. Firstly, 
we show that the mass stays "ahead" with probability of order $O(\epsilon)$ and secondly, that if it stays "ahead", then it has acquired a size of order $O(1)$ at the front.

We now give the mathematical framework for the coupling-techniques mentioned above. Let $T_{1}>0$ be arbitrarily fixed and $\theta_{c}<\underline{\theta} \leq \theta_{1}<\theta_{2} \leq \bar{\theta}<\infty$ with $\epsilon \equiv \theta_{2}-\theta_{1}>0$. For $u_{0} \in \mathcal{P}\left(\mathcal{C}_{\text {tem }}^{+}\right)$fixed, use a $\theta$-coupling to construct solutions $u_{s}^{(1)}(x)=u_{s}\left(\theta_{1}\right)(x), u_{s}^{(2)}(x)=u_{s}\left(\theta_{2}\right)(x), 0 \leq s \leq T_{1}$ to (1.1) such that $u_{s}^{(1)}(x) \leq u_{s}^{(2)}(x)$ for all $s \in\left[0, T_{1}\right], x \in \mathbb{R}$ a.s. solve

$$
u_{s}\left(\theta_{2}\right)(x)=u_{s}\left(\theta_{1}\right)(x)+v_{s}(x) \text { with } v_{s}(x) \geq 0 \text { for all } s \in\left[0, T_{1}\right], x \in \mathbb{R} \text { a.s. }
$$

and $v$ as in (5.24), that is, conditional on $\left.\sigma\left(u_{s}\left(\theta_{1}\right)\right): 0 \leq s \leq T_{1}\right)$, $v$ has distribution $Q^{0,\left(\theta_{2}-\theta_{1}\right) u\left(\theta_{1}\right), 2 u\left(\theta_{1}\right), 1}\left(\theta_{2}\right)$ (cf. (1.20) and Theorem 1.5) on $\left[0, T_{1}\right]$.

Let $T_{2}>0$ be arbitrarily fixed. Extend the above coupling to include a process $\left(w_{s}\right)_{s \in\left[0, T_{1}+T_{2}\right]}$ such that

$$
u_{s}\left(\theta_{1}\right)(x) \leq w_{s}(x) \leq u_{s}\left(\theta_{2}\right)(x) \text { for all } s \in\left[0, T_{1}+T_{2}\right], x \in \mathbb{R} \text { a.s. }
$$

as follows. Set

$$
w_{s}(x) \equiv u_{s}\left(\theta_{1}\right)(x)+v_{s}(x) \equiv \begin{cases}u_{s}^{\left(u_{0}\right)}\left(\theta_{2}\right)(x)=u_{s}^{\left(u_{0}\right)}\left(\theta_{1}\right)(x)+v_{s}(x) & \text { for } 0 \leq s \leq T_{1} \\ u_{s-T_{1}}^{\left(w_{T_{1}}\right)}\left(\theta_{1}\right)(x)=u_{s-T_{1}}^{\left(u_{T_{0}}^{\left(u_{0}\right)}\left(\theta_{1}\right)+v_{T_{1}}\right)}\left(\theta_{1}\right)(x) & \text { for } s \geq T_{1} .\end{cases}
$$

That is, conditional on $\mathcal{F}_{T_{1}}, w_{T_{1}+}$. has distribution $Q^{w_{T_{1}}, 0,0,1}\left(\theta_{1}\right)$. Indeed, to construct the coupling for the case $s>T_{1}$, condition on $\mathcal{F}_{T_{1}}$ and use a combination of a monotonicity-coupling and a $\theta$-coupling. To be more precise, use a monotonicity-coupling based on two independent white noises $W_{1}$, $W_{2}$ to construct

$$
u_{T_{1}+r}^{\left(u_{0}\right)}\left(\theta_{1}\right)(x)=u_{r}^{\left(u_{T_{1}}\left(\theta_{1}\right)\right)}\left(\theta_{1}\right)(x) \leq u_{r}^{\left(w_{T_{1}}\right)}\left(\theta_{1}\right)(x) \equiv u_{r}^{\left(u_{T_{1}}\left(\theta_{1}\right)\right)}\left(\theta_{1}\right)(x)+v_{T_{1}+r}(x) \text { for all } r \geq 0, x \in \mathbb{R}
$$

almost surely, with $v_{T_{1}+}$ solving (5.21). Then use a $\theta$-coupling to obtain

$$
u_{r}^{\left(w_{T_{1}}\right)}\left(\theta_{1}\right)(x) \leq u_{r}^{\left(w_{T_{1}}\right)}\left(\theta_{2}\right)(x) \equiv u_{r}^{\left(w_{T_{1}}\right)}\left(\theta_{1}\right)(x)+\hat{v}_{T_{1}+r}(x) \text { for all } r \geq 0, x \in \mathbb{R}
$$

almost surely, where the difference process $\hat{v}$ solves (5.24) with a white noise $W_{3}$ independent of $W_{1}, W_{2}$ from above. As a result,

$$
\begin{aligned}
u_{T_{1}+r}^{\left(u_{0}\right)}\left(\theta_{1}\right)(x) & \leq u_{T_{1}+r}^{\left(u_{0}\right)}\left(\theta_{1}\right)(x)+v_{T_{1}+r}(x)=w_{T_{1}+r}(x) \\
& \leq u_{T_{1}+r}^{\left(u_{0}\right)}\left(\theta_{1}\right)(x)+v_{T_{1}+r}(x)+\hat{v}_{T_{1}+r}(x)=u_{r}^{\left(w_{T_{1}}\right)}\left(\theta_{2}\right)(x)=u_{r}^{\left(u_{T_{1}}^{\left(u_{0}\right)}\left(\theta_{2}\right)\right)}\left(\theta_{2}\right)(x)=u_{T_{1}+r}^{\left(u_{0}\right)}\left(\theta_{2}\right)(x)
\end{aligned}
$$

holds indeed true.

\subsection{A first estimate}

The following estimate is fundamental in the first step of the construction. Recall (3.3) and thus compare the following SPDE with (5.24) from the $\theta$-coupling which quantifies the gain in density due to an increase in $\theta$.

Let

$$
\Upsilon=\left\{f \in \mathcal{C}(\mathbb{R}, \mathbb{R}):\|f\|_{\lambda}=\sup \{|f(x)| \exp (-\lambda|x|): x \in \mathbb{R}\}<\infty \text { for some } \lambda<0\right\}
$$

be the set of continuous functions with exponential decay. For existence and uniqueness of solutions to all of the SPDEs mentioned in the proof below, see Theorem 1.5 .

Also let

$$
\tilde{\Upsilon} \equiv\left\{\psi \in \mathcal{C}^{1,2} \text { and } \sup _{t \in[0, T]}\left|\psi_{t}(\cdot)\right| \wedge\left|\frac{\partial \psi_{t}(\cdot)}{\partial t}\right| \wedge\left|\Delta \psi_{t}(\cdot)\right| \in \Upsilon\right\} .
$$


Lemma 3.1. Let $T>0, \zeta \in \mathcal{C}\left([0, T], \mathcal{C}_{\text {tem }}^{+}\right) \backslash\{0\}, W$ a white noise and $\epsilon>0$ be arbitrarily fixed. Let $v=v(\epsilon, \theta, \zeta)$ be a solution to

$$
\frac{\partial v}{\partial t}=\Delta v+\epsilon \zeta+(\theta-v-2 \zeta) v+\sqrt{v} \dot{W}, \quad v(0)=0, \quad t \in[0, T] .
$$

For $g \in \Upsilon, g \geq 0, g \not \equiv 0$ fixed,

$$
0<c_{-}(\zeta, g, \theta, T)=\liminf _{\epsilon \downarrow 0^{+}} \frac{\mathbb{E}\left[1-e^{-2\left\langle v_{T}, g\right\rangle}\right]}{\epsilon} \leq \limsup _{\epsilon \downarrow 0^{+}} \frac{\mathbb{E}\left[1-e^{-2\left\langle v_{T}, g\right\rangle}\right]}{\epsilon}=c_{+}(\zeta, g, \theta, T)<\infty
$$

holds true.

Proof. The lower bound. Fix $\zeta, g, \theta$ and $T$ as above. Let

$$
I(\epsilon)=\mathbb{E}\left[1-e^{-2\left\langle v_{T}, g\right\rangle}\right] .
$$

Subsequently, dominate $v=v(\epsilon)$ by the sum of two independent solutions (cf. the construction of the coupling with two independent processes in Remark 5.11 below) satisfying

$$
\frac{\partial v^{(i)}}{\partial t}=\Delta v^{(i)}+\frac{\epsilon}{2} \zeta+\left(\theta-v^{(i)}-2 \zeta\right) v^{(i)}+\sqrt{v^{(i)}} \dot{W}_{i}, \quad v^{(i)}(0)=0, \quad i=1,2, \quad t \geq 0
$$

such that $v(t, x) \leq v^{(1)}(t, x)+v^{(2)}(t, x)$ for all $t \geq 0, x \in \mathbb{R}$ a.s. Note that $v^{(i)}=v^{(i)}(\epsilon) \stackrel{\mathcal{D}}{=} v(\epsilon / 2), i=1,2$. We obtain by the independence and the identical distribution of the two non-negative solutions, for all $\epsilon, T>0$,

$$
I(\epsilon) \leq \mathbb{E}\left[1-e^{-2\left\langle v_{T}^{(1)}+v_{T}^{(2)}, g\right\rangle}\right]=\mathbb{E}\left[1-e^{-2\left\langle v_{T}^{(1)}, g\right\rangle}\right] \mathbb{E}\left[1+e^{-2\left\langle v_{T}^{(2)}, g\right\rangle}\right] \leq 2 I(\epsilon / 2) \Longleftrightarrow\left(\frac{I(\epsilon)}{\epsilon} \leq \frac{I(\epsilon / 2)}{\epsilon / 2}\right) .
$$

By Theorem 1.5b), $I(\epsilon)$ is continuous in $\epsilon$. Hence, to establish the lower bound, it is enough to show that there exists $\epsilon_{0}>0$ such that $I(\epsilon)>0$ for all $\epsilon \in\left[\epsilon_{0}, 2 \epsilon_{0}\right]$. Indeed, by the continuity of $I$ and (3.15), it then follows that

$$
\inf _{\epsilon \in\left(0,2 \epsilon_{0}\right]} \frac{I(\epsilon)}{\epsilon} \geq \inf _{\epsilon \in\left[\epsilon_{0}, 2 \epsilon_{0}\right]} \frac{I(\epsilon)}{\epsilon}>0 .
$$

By reasoning as for an immigration-coupling, it follows that $I(\epsilon)$ is monotonically increasing in $\epsilon$. It is therefore enough to find $\epsilon_{0}=\epsilon_{0}(T)>0$ such that $I\left(\epsilon_{0}\right)>0$. By definition of $v$, this holds true for arbitrary $T>0$. Indeed, use for instance Theorem 1.5c) to see that with $\mathbb{P}(v)$ denoting the distribution of $v, \mathbb{P}(v)=Q^{0, \epsilon \zeta, 2 \zeta, 1}$ and $Q^{0, \epsilon \zeta, 0,0}$ are mutually absolutely continuous on $\mathcal{U}_{R, T}$ (recall the notation from Theorem [1.5) for $R, T>0$ arbitrarily fixed. Here, the law $Q^{0, \epsilon \zeta, 0,0}$ is the law of the solution to

$$
\frac{\partial w}{\partial t}=\Delta w+\epsilon \zeta+\theta w+\sqrt{w} \dot{W}, \quad w(0)=0, \quad t \geq 0 .
$$

The latter is a superprocess with immigration and thus satisfies $\mathbb{P}\left(\left\langle w_{T}, g\right\rangle>0\right)>0$.

The upper bound. We now derive the upper bound in (3.12). Couple a solution $v$ of (3.11) with a solution $V$ of

$$
\frac{\partial V}{\partial t}=\Delta V+\epsilon \zeta+\theta V+\sqrt{V} \dot{W}_{3}, \quad V(0)=0, \quad t \geq 0,
$$

such that $v(t, x) \leq V(t, x)$ for all $t \geq 0, x \in \mathbb{R}$ a.s. Here, $W_{3}$ is an appropriate white noise and we use techniques as in Subsection 5.3 of the appendix. See the beginning of [19, Section 2] for the theoretical background of what follows. We obtain for test functions $\psi(t, x)=\psi_{t}(x), t \geq 0, x \in \mathbb{R}$ satisfying $\psi \in \tilde{\Upsilon}$ and for $t \geq 0$ arbitrary, by an application of Itô's formula,

$$
e^{-2\left\langle V_{t}, \psi_{t}\right\rangle}=1+M_{t}-2 \int_{0}^{t} e^{-2\left\langle V_{s}, \psi_{s}\right\rangle}\left\{\epsilon\left\langle\zeta_{s}, \psi_{s}\right\rangle+\left\langle V_{s}, \frac{\partial \psi_{s}}{\partial s}+\Delta \psi_{s}+\theta \psi_{s}-\frac{2}{2} \psi_{s}^{2}\right\rangle\right\} d s,
$$


where $\left(M_{t}\right)_{t \geq 0}$ is a local martingale with quadratic variation

$$
\langle M .\rangle_{t}=4 \int_{0}^{t} \int e^{-4\left\langle V_{s}, \psi_{s}\right\rangle} V_{s}(x) \psi_{s}^{2}(x) d x d s .
$$

For $T>0$ fixed and $0 \leq s \leq T$, choose $\psi(s, z) \equiv \Psi(T-s, z)$, where $\Psi(s, x)=\Psi_{s}(x)$ is the unique non-negative solution to the partial differential equation (PDE)

$$
\frac{\partial \Psi_{s}}{\partial s}=\Delta \Psi_{s}+\theta \Psi_{s}-\Psi_{s}^{2}, \quad \Psi_{0}=g, \quad 0 \leq s \leq T
$$

(cf. Iscoe [10, Theorem A of the appendix] with $A \psi=\Delta \psi+\theta \psi, g(x)=x^{2}$ and $\mathcal{D}(A)=\left\{f \in \mathcal{C}^{2}(\mathbb{R}, \mathbb{R})\right.$ : $f, \Delta f \in \Upsilon)$. Then $\psi \in \tilde{\Upsilon}$ and we obtain for $0 \leq t \leq T$,

$$
e^{-2\left\langle V_{t}, \psi_{t}\right\rangle}=1+M_{t}-2 \epsilon \int_{0}^{t} e^{-2\left\langle V_{s}, \psi_{s}\right\rangle}\left\langle\zeta_{s}, \psi_{s}\right\rangle d s
$$

Note that the integral on the right hand side is finite as $\zeta \in \mathcal{C}\left([0, \infty), \mathcal{C}_{\text {tem }}^{+}\right)$and $\sup _{s \in[0, T]}\left|\psi_{s}(\cdot)\right| \in \Upsilon$. Let $t=T$. In case $\left(M_{t}\right)_{t \in[0, T]}$ is a martingale, take expectations to conclude

$$
\mathbb{E}\left[1-e^{-2\left\langle V_{T}, g\right\rangle}\right]=2 \epsilon \int_{0}^{T} \mathbb{E}\left[e^{-2\left\langle V_{s}, \psi_{s}\right\rangle}\left\langle\zeta_{s}, \psi_{s}\right\rangle\right] d s
$$

In case $\left(M_{t}\right)_{t \in[0, T]}$ is only a local martingale, take a sequence of increasing stopping times $\tau_{n} \uparrow T$ such that $\left(M_{t \wedge \tau_{n}}\right)_{t \in[0, T]}$ is a martingale for each $n \in \mathbb{N}$ fixed. Take expectations and subsequently use dominated convergence to obtain the same conclusion.

The coupling of $v$ and $V$ yields

$$
\mathbb{E}\left[1-e^{-2\left\langle v_{T}, g\right\rangle}\right] \leq \mathbb{E}\left[1-e^{-2\left\langle V_{T}, g\right\rangle}\right]=2 \epsilon \int_{0}^{T} \mathbb{E}\left[e^{-2\left\langle V_{s}, \psi_{s}\right\rangle}\left\langle\zeta_{s}, \psi_{s}\right\rangle\right] d s \leq 2 \epsilon \int_{0}^{T}\left\langle\zeta_{s}, \Psi_{T-s}\right\rangle d s
$$

It thus remains to show that $\int_{0}^{T}\left\langle\zeta_{s}, \Psi_{T-s}\right\rangle d s<\infty$. The latter follows from the assumption $\zeta \in$ $\mathcal{C}\left([0, \infty), \mathcal{C}_{\text {tem }}^{+}\right)$and as $\left(\psi_{s}\right)_{s \in[0, T]}=\left(\Psi_{T-s}\right)_{s \in[0, T]}$ satisfies (3.10).

\subsection{Increase of the right marker}

We now follow the strategy as outlined in Subsection 3.1. We start by investigating the increase of the right marker of a solution due to an increase in $\theta$.

Let $f \in \mathcal{C}_{\text {tem }}^{+}$with $R_{0}(f)<\infty$ and $\mathbb{P}_{f}(\tau=\infty)=1$. Recall the notation from Subsection 3.1 . in particular the definition of $u, v, w=u^{(f)}\left(\theta_{1}\right)+v$ with $u_{0}=f$ from (3.5). In what follows, write $u .(x)=u^{(f)}\left(\theta_{1}\right)(x)$ and set $\mathcal{F}_{T}^{u}=\sigma\left(u_{t}: 0 \leq t \leq T\right)$ for $T>0$ arbitrary. Note that in the proofs to follow we will often only write $\mathbb{E}$ or $\mathbb{P}$ when the context is clear. In the main statements, the indices are kept however. This will allow us to avoid changes in indexing when using duality relations.

Lemma 3.2. Let $T_{1}, T_{2}>0$ be arbitrarily fixed and $\theta_{c}<\underline{\theta} \leq \theta_{1}<\theta_{2} \leq \bar{\theta}$. For all $\delta^{\prime}>0$ there exists $\eta_{1}=\eta_{1}\left(\delta^{\prime}, T_{1}, T_{2}, \underline{\theta}, \bar{\theta}\right)>0$ small enough such that

$$
\int_{\mathcal{C}_{\text {tem }}^{+}} \mathbb{P}_{f}\left(\mathbb{E}_{f}\left[0 \vee\left(R_{0}\left(w_{T_{1}+T_{2}}\right) \wedge 1\right)-0 \vee\left(R_{0}\left(u_{T_{1}+T_{2}}\right) \wedge 1\right) \mid \mathcal{F}_{T_{1}}^{u}\right] \geq \eta_{1}\left(\theta_{2}-\theta_{1}\right)\right)\left(\nu_{T}^{*, l}\left(\theta_{1}\right)\right)(d f) \geq 1-2 \delta^{\prime}
$$

for all $T>1$.

Remark 3.3. Note that $\mathbb{P}_{\nu_{T}^{*, l}\left(\theta_{1}\right)}(\tau=\infty)=1$ by (5.8) and Remark 2.6 . 
Proof. Recall $Q^{f, \alpha, \beta, \gamma} \equiv Q_{u}^{f, \alpha, \beta, \gamma}(\theta)$ from Notation 1.4 and Theorem 1.5. With regards to the following conditional expectation $\mathbb{E}\left[0 \vee\left(R_{0}\left(w_{T_{1}+T_{2}}\right) \wedge 1\right)-0 \vee\left(R_{0}\left(u_{T_{1}+T_{2}}\right) \wedge 1\right) \mid \mathcal{F}_{T_{1}}^{u}\right]$, recall that for the coupling from (3.5), the difference $\left(0 \vee\left(R_{0}\left(w_{t}\right) \wedge 1\right)\right)-\left(0 \vee\left(R_{0}\left(u_{t}\right) \wedge 1\right)\right)$ is non-negative for all $t \geq 0$ almost surely. Moreover, conditional on $\mathcal{F}_{T_{1}}^{u}, v$ has law $Q^{0,\left(\theta_{2}-\theta_{1}\right) u, 2 u, 1}\left(\theta_{2}\right) \equiv Q_{v}^{0,\left(\theta_{2}-\theta_{1}\right) u, 2 u, 1}\left(\theta_{2}\right)$ on $\left[0, T_{1}\right]$ and conditional on $\mathcal{F}_{T_{1}}, w$ has law $Q^{u_{T_{1}}+v_{T_{1}}, 0,0,1}\left(\theta_{1}\right) \equiv Q_{w}^{u_{T_{1}}+v_{T_{1}}, 0,0,1}\left(\theta_{1}\right)$ on $\left[T_{1}, T_{1}+T_{2}\right]$. Thus, as the laws $Q^{f, \alpha, \beta, \gamma}$ for $f \in \mathcal{C}_{\text {tem }}^{+}$form a strong Markov family by Theorem $1.5 \mathrm{~b}$ ), and by (3.4),

$$
\begin{aligned}
& \mathbb{E}\left[0 \vee\left(R_{0}\left(w_{T_{1}+T_{2}}\right) \wedge 1\right)-0 \vee\left(R_{0}\left(u_{T_{1}+T_{2}}\right) \wedge 1\right) \mid \mathcal{F}_{T_{1}}^{u}\right] \\
& =Q_{v}^{0,\left(\theta_{2}-\theta_{1}\right) u, 2 u, 1}\left(\theta_{2}\right)\left[Q_{w}^{u_{T_{1}}+v_{T_{1}}, 0,0,1}\left(\theta_{1}\right)\left[0 \vee\left(R_{0}\left(u_{T_{2}}\right) \wedge 1\right)\right]\right]-Q_{u}^{u_{T_{1}}, 0,0,1}\left(\theta_{1}\right)\left[0 \vee\left(R_{0}\left(u_{T_{2}}\right) \wedge 1\right)\right] \text { a.s. }
\end{aligned}
$$

Note that by Remark 3.3, $u$ survives almost surely. Recall (2.39)-(2.41) to rewrite

$$
\begin{aligned}
& \mathbb{E}\left[0 \vee\left(R_{0}\left(w_{T_{1}+T_{2}}\right) \wedge 1\right)-0 \vee\left(R_{0}\left(u_{T_{1}+T_{2}}\right) \wedge 1\right) \mid \mathcal{F}_{T_{1}}^{u}\right] \\
& =\int_{0}^{1} \mathbb{P}\left(R_{0}\left(w_{T_{1}+T_{2}}\right)>x \mid \mathcal{F}_{T_{1}}^{u}\right) d x-\int_{0}^{1} \mathbb{P}\left(R_{0}\left(u_{T_{1}+T_{2}}\right)>x \mid \mathcal{F}_{T_{1}}^{u}\right) d x \\
& =\int_{0}^{1} \mathbb{E}\left[1-e^{-2\left\langle w_{T_{1}}(\cdot+x), u_{T_{2}}^{*, r}\left(\theta_{1}\right)\right\rangle} \mid \mathcal{F}_{T_{1}}^{u}\right] d x-\int_{0}^{1} \mathbb{E}\left[1-e^{-2\left\langle u_{T_{1}}(\cdot+x), u_{T_{2}}^{*, r}\left(\theta_{1}\right)\right\rangle} \mid \mathcal{F}_{T_{1}}^{u}\right] d x \\
& =\int_{0}^{1} \mathbb{E}\left[e^{-2\left\langle u_{T_{1}}(\cdot+x), u_{T_{2}}^{*, r}\left(\theta_{1}\right)\right\rangle}\left(1-e^{-2\left\langle v_{T_{1}}(\cdot+x), u_{T_{2}}^{*, r}\left(\theta_{1}\right)\right\rangle}\right) \mid \mathcal{F}_{T_{1}}^{u}\right] d x .
\end{aligned}
$$

Use a $\theta$-*-coupling to conclude that this is bounded below by

$$
\begin{aligned}
& \int_{0}^{1} \mathbb{E}\left[e^{-2\left\langle u_{T_{1}}(\cdot+x), u_{T_{2}}^{*, r}(\bar{\theta})\right\rangle}\left(1-e^{-2\left\langle v_{T_{1}}(\cdot+x), u_{T_{2}}^{*, r}(\underline{\theta})\right\rangle}\right) \mid \mathcal{F}_{T_{1}}^{u}\right] d x \\
& =\int_{0}^{1} \mathbb{E}\left[e^{-2\left\langle u_{T_{1}}(\cdot+x), u_{T_{2}}^{*, r}(\bar{\theta})\right\rangle_{1}} \mathbb{E}\left[1-e^{-2\left\langle v_{T_{1}}(\cdot+x), u_{T_{2}}^{*, r}(\underline{\theta})\right\rangle} \mid \sigma\left(\mathcal{F}^{u^{*, r}}, \mathcal{F}_{T_{1}}^{u}\right)\right] \mid \mathcal{F}_{T_{1}}^{u}\right] d x,
\end{aligned}
$$

where we let $\mathcal{F}^{u^{*, r}}=\sigma\left(u_{t}^{*, r}(\underline{\theta}), u_{t}^{*, r}(\bar{\theta}): t \geq 0\right)$.

Let $\epsilon=\theta_{2}-\theta_{1}$. We now randomize the initial condition. Recall $\nu_{T}^{*, l}\left(\theta_{1}\right)$ as defined in (5.8) of the appendix. Let $\eta_{1}, T>0$ be arbitrarily fixed. The quantity we are interested in is

$$
I_{1} \equiv \int_{\mathcal{C}_{\text {tem }}^{+}} \mathbb{P}_{f}\left(\mathbb{E}\left[0 \vee\left(R_{0}\left(w_{T_{1}+T_{2}}\right) \wedge 1\right)-0 \vee\left(R_{0}\left(u_{T_{1}+T_{2}}\right) \wedge 1\right) \mid \mathcal{F}_{T_{1}}^{u}\right] \geq \eta_{1} \epsilon\right)\left(\nu_{T}^{*, l}\left(\theta_{1}\right)\right)(d f)
$$

We get with the help of (3.27)-(3.28) as a lower bound to (3.29),

$$
\left.\int_{\mathcal{C}_{\text {tem }}^{+}} \mathbb{P}_{f}\left(\int_{0}^{1} \mathbb{E}\left[e^{-2\left\langle u_{T_{1}}(\cdot+x), u_{T_{2}}^{*, r}(\bar{\theta})\right.}\right\rangle_{\mathbb{E}}\left[1-e^{-2\left\langle v_{T_{1}}(\cdot+x), u_{T_{2}}^{*, r}(\underline{\theta})\right\rangle} \mid \sigma\left(\mathcal{F}^{u^{*, r}}, \mathcal{F}_{T_{1}}^{u}\right)\right] \mid \mathcal{F}_{T_{1}}^{u}\right] d x \geq \eta_{1} \epsilon\right)\left(\nu_{T}^{*, l}\left(\theta_{1}\right)\right)(d f) .
$$

Here we note that $\left(v_{t}\right)_{t \in\left[0, T_{1}\right]}$ solves (5.24) respectively (3.11) with $\theta=\theta_{2}$ and $\left(\zeta_{t}\right)_{t \in\left[0, T_{1}\right]}=\left(u_{t}\right)_{t \in\left[0, T_{1}\right]}=$ $\left(u_{t}^{(f)}\right)\left(\theta_{1}\right)_{t \in\left[0, T_{1}\right]}$ and $f$ drawn according to $\nu_{T}^{*, l}\left(\theta_{1}\right)$. By (5.11) and Corollary 5.5, for every $\delta^{\prime}>0$ there exist a compact set $K_{\delta^{\prime}} \subset \mathcal{C}_{\text {tem }}^{+}$and $d_{0}=d_{0}\left(\delta^{\prime}\right), m_{0}=m_{0}\left(\delta^{\prime}\right)>0$ such that

$$
\inf _{\underline{\theta} \leq \theta \leq \bar{\theta}}\left(\nu_{T}^{*, l}(\theta)\right)\left(K_{\delta^{\prime}} \cap M\left(d_{0}, m_{0}\right)\right) \geq 1-\delta^{\prime} \quad \text { for all } T>1
$$

with

$$
M\left(d_{0}, m_{0}\right) \equiv\left\{f \in \mathcal{C}_{\text {tem }}^{+}: \text {there exist }-1 / 2 \leq l_{0}<r_{0} \leq 0 \text { with }\left|r_{0}-l_{0}\right|=d_{0} \text { such that } f \geq m_{0} \mathbb{1}_{\left[l_{0}, r_{0}\right]}\right\}
$$


for $d_{0}, m_{0}>0$. Observe first that $M\left(d_{0}, m_{0}\right) \cap K_{\delta^{\prime}}$ is compact in $\mathcal{C}_{\text {tem }}^{+}$. Indeed, use that if $\left(f_{n}\right)_{n} \subset$ $M\left(d_{0}, m_{0}\right) \cap K_{\delta^{\prime}}$, then there exists a subsequence $\left(f_{n_{k}}\right)_{k} \subset M\left(d_{0}, m_{0}\right)$ that converges to a limit in $K_{\delta^{\prime}}$. Let $x_{n_{k}}=l_{n_{k}}+d_{0} / 2$ such that $f_{n_{k}} \geq m_{0} \mathbb{1}_{\left[x_{n_{k}}-d_{0} / 2, x_{n_{k}}+d_{0} / 2\right]}$ and $l_{n_{k}} \leq x_{n_{k}} \leq r_{n_{k}},\left|r_{n_{k}}-l_{n_{k}}\right|=d_{0}, l_{n_{k}}, r_{n_{k}} \in$ $[-1 / 2,0]$. By the compactness of $[-1 / 2,0]$ there exists a subsequence $x_{n_{k_{l}}} \rightarrow x_{0} \in[-1 / 2,0]$ for $l \rightarrow \infty$ and as a result, $f_{n_{k_{l}}}$ converges to a limit in $M\left(d_{0}, m_{0}\right) \cap K_{\delta^{\prime}}$.

Conditional on $\sigma\left(\mathcal{F}^{u^{*, r}}, \mathcal{F}_{T_{1}}^{u}\right)$, we now apply the lower bound of Lemma 3.1 for some $0 \not \equiv g=g_{x} \in$ $\Upsilon, 0 \leq g \leq u_{T_{2}}^{*, r}(\underline{\theta})(\cdot-x)$. Recall that $u_{t}=u_{t}\left(\theta_{1}\right)$. From below (3.16) it follows that it is enough to show for $\epsilon_{0}>0$ arbitrarily fixed that

$$
\begin{aligned}
& \quad \inf _{\theta_{1} \in[\underline{\theta}, \bar{\theta}]} \int_{K_{\delta^{\prime}} \cap M\left(d_{0}, m_{0}\right)} \mathbb{P}_{f}\left(\int _ { 0 } ^ { 1 } \mathbb { E } \left[e^{-2\left\langle u_{T_{1}}(\cdot+x), u_{T_{2}}^{*, r}(\bar{\theta})\right\rangle}\right.\right. \\
& \left.\left.\quad \times \mathbb{E}\left[1-e^{-2\left\langle v_{T_{1}}\left(\epsilon_{0}, \theta_{2},\left(u_{t}\right)_{t \in\left[0, T_{1}\right]}\right), g_{x}\right\rangle} \mid \sigma\left(\mathcal{F}^{u^{*, r}}, \mathcal{F}_{T_{1}}^{u}\right)\right] \mid \mathcal{F}_{T_{1}}^{u}\right] d x \geq \eta_{1} \epsilon_{0}\right)\left(\nu_{T}^{*, l}\left(\theta_{1}\right)\right)(d f) \geq 1-2 \delta^{\prime}
\end{aligned}
$$

for $\eta_{1}$ small enough and $\theta_{2}=\theta_{1}+\epsilon_{0}$. The left hand side in the above can be bounded from below by

$$
\begin{aligned}
\left(1-\delta^{\prime}\right) \inf _{\theta \in[\underline{\theta}, \bar{\theta}]} & \inf _{f \in K_{\delta^{\prime}} \cap M\left(d_{0}, m_{0}\right)} \mathbb{P}_{f}\left(\int _ { 0 } ^ { 1 } \mathbb { E } \left[e^{-2\left\langle u_{T_{1}}(\cdot+x), u_{T_{2}}^{*, r}(\bar{\theta})\right\rangle}\right.\right. \\
& \left.\left.\times \mathbb{E}\left[1-e^{-2\left\langle v_{T_{1}}\left(\epsilon_{0}, \theta_{2},\left(u_{t}\right)_{t \in\left[0, T_{1}\right]}\right), g_{x}\right\rangle} \mid \sigma\left(\mathcal{F}^{u^{*, r}}, \mathcal{F}_{T_{1}}^{u}\right)\right] \mid \mathcal{F}_{T_{1}}^{u}\right] d x \geq \eta_{1} \epsilon_{0}\right),
\end{aligned}
$$

where we used (3.31).

The map $(f, \alpha, \beta, 1) \mapsto Q^{f, \alpha, \beta, 1}$ is continuous by Theorem[1.5b). Hence, the law $\mathbb{P}_{f}\left(\theta_{1}\right)=Q^{f, 0,0,1}\left(\theta_{1}\right)=$ $Q^{f, 0,\left(\bar{\theta}-\theta_{1}\right), 1}(\bar{\theta})$ of $u$ is continuous in $f$ and $\theta_{1}$. Furthermore, by the continuous mapping theorem, the law of $v\left(\epsilon_{0}, \theta_{2}, u\right)$, that is $Q^{0, \epsilon_{0} u, 2 u, 1}\left(\theta_{1}+\epsilon_{0}\right)=Q^{0, \epsilon_{0} u, 2 u+\left(\bar{\theta}-\theta_{1}\right), 1}\left(\bar{\theta}+\epsilon_{0}\right)$ is also continuous in $f$ and $\theta_{1}$. As $[\underline{\theta}, \bar{\theta}]$ is a compact interval and $M\left(d_{0}, m_{0}\right) \cap K_{\delta^{\prime}}$ is compact in $\mathcal{C}_{\text {tem }}^{+}$, the infimum is attained for some $\theta^{\prime} \in[\underline{\theta}, \bar{\theta}], f^{\prime} \in M\left(d_{0}, m_{0}\right) \cap K_{\delta^{\prime}}$. Let $\theta^{\prime}, f^{\prime}$ be arbitrarily fixed. The innermost expectation is non-zero almost surely by reasoning as in (3.17) of the proof of the lower bound in Lemma 3.1. Let $x \in[0,1]$ be arbitrarily fixed. Then (1.11) and symmetry yield for $u_{t}=u_{t}^{\left(f^{\prime}\right)}\left(\theta^{\prime}\right)$,

$\mathbb{E}\left[e^{-2\left\langle u_{T_{1}}(\cdot+x), u_{T_{2}}^{*, r}(\bar{\theta})\right\rangle} \mid \mathcal{F}_{T_{1}}^{u}\right]=\mathbb{P}\left(\left\langle\mathbb{1}_{(0, \infty)}(\cdot), u_{T_{2}}^{\left(u_{T_{1}}(\cdot+x)\right)}(\bar{\theta})\right\rangle=0 \mid \mathcal{F}_{T_{1}}^{u}\right)=\mathbb{P}\left(R_{0}\left(u_{T_{2}}^{\left(u_{T_{1}}(\cdot+x)\right)}(\bar{\theta})\right) \leq 0 \mid \mathcal{F}_{T_{1}}^{u}\right)$.

The latter is non-zero almost surely. Thus, using dominated convergence, we can choose $\eta_{1}>0$ small enough such that $I_{1} \geq\left(1-\delta^{\prime}\right)^{2} \geq 1-2 \delta^{\prime}$.

Corollary 3.4. Let $T_{1}, T_{2}>0$ be arbitrarily fixed and $\theta_{c}<\underline{\theta} \leq \theta_{1}<\theta_{2} \leq \bar{\theta}$. For all $\delta^{\prime}>0$ there exists $\eta_{1}=\eta_{1}\left(\delta^{\prime}, T_{1}, T_{2}, \underline{\theta}, \bar{\theta}\right)>0$ small enough such that

$$
\int_{\mathcal{C}_{\text {tem }}^{+}} \mathbb{P}_{f}\left(\mathbb{P}_{f}\left(R_{0}\left(w_{T_{1}+T_{2}}\right)>R_{0}\left(u_{T_{1}+T_{2}}\right) \mid \mathcal{F}_{T_{1}}^{u}\right) \geq \eta_{1}\left(\theta_{2}-\theta_{1}\right)\right)\left(\nu_{T}^{*, l}\left(\theta_{1}\right)\right)(d f) \geq 1-2 \delta^{\prime}
$$

for all $T>1$.

Proof. Use that $\mathbb{1}_{\{X>Y\}} \geq 0 \vee(X \wedge 1)-0 \vee(Y \wedge 1)$ for $X \geq Y$.

Lemma 3.5. Let $T_{1}, T_{2}>0$ be arbitrarily fixed and $\theta_{c}<\underline{\theta} \leq \theta_{1}<\theta_{2} \leq \bar{\theta}$. For all $\delta^{\prime}>0$ there exists $\eta_{2}=\eta_{2}\left(\delta^{\prime}, T_{1}, T_{2}, \underline{\theta}, \bar{\theta}\right)>0$ big enough such that

$$
\int_{\mathcal{C}_{\text {tem }}^{+}} \mathbb{P}_{f}\left(\mathbb{P}_{f}\left(R_{0}\left(w_{T_{1}+T_{2}}\right)>R_{0}\left(u_{T_{1}+T_{2}}\right) \mid \mathcal{F}_{T_{1}}^{u}\right) \leq \eta_{2}\left(\theta_{2}-\theta_{1}\right)\right)\left(\nu_{T}^{*, l}\left(\theta_{1}\right)\right)(d f) \geq 1-4 \delta^{\prime}
$$

for all $T>1$. 
Proof. First note that $w=u+v$ as in (3.5) and thus

$$
\mathbb{P}\left(R_{0}\left(w_{T_{1}+T_{2}}\right)>R_{0}\left(u_{T_{1}+T_{2}}\right) \mid \mathcal{F}_{T_{1}}^{u}\right)=\mathbb{P}\left(R_{0}\left(v_{T_{1}+T_{2}}\right)>R_{0}\left(u_{T_{1}+T_{2}}\right) \mid \mathcal{F}_{T_{1}}^{u}\right) .
$$

Recall the construction of $v_{T_{1}+r}, r \in\left[0, T_{2}\right]$ by means of a monotonicity-coupling from (3.6). Extend this coupling as follows.

$$
\begin{array}{lll}
\frac{\partial u}{\partial t}=\Delta u+\left(\theta_{1}-u\right) u+\sqrt{u} \dot{W}_{1}, & u\left(T_{1}\right)=u_{T_{1}}^{\left(u_{0}\right)}\left(\theta_{1}\right), \\
\frac{\partial v}{\partial t}=\Delta v+\left(\theta_{1}-v-2 u\right) v+\sqrt{v} \dot{W}_{2}, & v\left(T_{1}\right)=v_{T_{1}}=w_{T_{1}}-u_{T_{1}}^{\left(u_{0}\right)}\left(\theta_{1}\right), \\
\frac{\partial d}{\partial t}=\Delta d+2 u v+\left(\theta_{1}-d-2 v\right) d+\sqrt{d} \dot{W}_{3}, & d\left(T_{1}\right)=0,
\end{array}
$$

$t \geq T_{1}$, with $W_{i}, i=1,2,3$ independent white noises. Then $U \equiv v+d$ solves, conditional on $\mathcal{F}_{T_{1}+T_{2}}^{u}$,

$$
\frac{\partial U}{\partial t}=\Delta U+\left(\theta_{1}-U\right) U+\sqrt{d} \dot{W}_{4}, \quad U\left(T_{1}\right)=v_{T_{1}}, \quad t \geq T_{1}
$$

for some white noise $W_{4}$ independent of $W_{1}$. By construction, $v_{T_{1}+t}(x) \leq U_{T_{1}+t}(x)$ for all $x \in \mathbb{R}, t \in\left[0, T_{2}\right]$ almost surely and the law of $U$ only depends on $\mathcal{F}_{T_{1}}^{u}$ through the initial condition.

Now reason similarly to (3.27)-(3.28) to obtain

$$
\begin{aligned}
& \mathbb{P}\left(R_{0}\left(v_{T_{1}+T_{2}}\right)>R_{0}\left(u_{T_{1}+T_{2}}\right) \mid \mathcal{F}_{T_{1}}^{u}\right) \leq \mathbb{P}\left(R_{0}\left(U_{T_{1}+T_{2}}\right)>R_{0}\left(u_{T_{1}+T_{2}}\right) \mid \mathcal{F}_{T_{1}}^{u}\right) \\
& =\mathbb{P}\left(\mathbb{P}\left(R_{0}\left(U_{T_{1}+T_{2}}\right)>R_{0}\left(u_{T_{1}+T_{2}}\right) \mid \mathcal{F}_{T_{1}+T_{2}}^{u}\right) \mid \mathcal{F}_{T_{1}}^{u}\right) \\
& =\mathbb{E}\left[\mathbb{E}\left[1-e^{-2\left\langle U_{T_{1}}\left(\cdot+R_{0}\left(u_{T_{1}+T_{2}}\right)\right), u_{T_{2}}^{*, r}\left(\theta_{1}\right)\right\rangle} \mid \mathcal{F}_{T_{1}+T_{2}}^{u}\right] \mid \mathcal{F}_{T_{1}}^{u}\right] \\
& =\mathbb{E}\left[1-e^{-2\left\langle v_{T_{1}}\left(\cdot+R_{0}\left(u_{T_{1}+T_{2}}\right)\right), u_{T_{2}}^{*, r}\left(\theta_{1}\right)\right\rangle} \mid \mathcal{F}_{T_{1}}^{u}\right] \\
& \leq \mathbb{E}\left[1-e^{-2\left\langle v_{T_{1}}\left(\cdot+R_{0}\left(u_{T_{1}+T_{2}}\right)\right), u_{T_{2}}^{*, r}(\bar{\theta})\right\rangle} \mid \mathcal{F}_{T_{1}}^{u}\right] \\
& =\mathbb{E}\left[\mathbb { E } \left[1-e^{\left.\left.-2\left\langle v_{T_{1}},\left(u_{T_{2}}^{*, r}(\bar{\theta})\right)\left(\cdot-R_{0}\left(u_{T_{1}+T_{2}}\right)\right)\right\rangle \mid \sigma\left(\mathcal{F}^{u^{*, r}}, \mathcal{F}_{T_{1}}^{u}\right)\right] \mid \mathcal{F}_{T_{1}}^{u}\right] .}\right.\right.
\end{aligned}
$$

In the third equality we used that $U\left(T_{1}\right)=v_{T_{1}}$.

Use Lemma 5.2 to obtain that for all $\theta \in[\underline{\theta}, \bar{\theta}], T_{1}, T_{2}>0, A>0, T \geq 1$,

$$
\mathbb{P}_{\nu_{T}^{*, l}(\theta)}\left(\left|R_{0}\left(u_{T_{1}+T_{2}}\right)\right| \geq A\right) \leq \frac{C\left(\underline{\theta}, \bar{\theta}, T_{1}+T_{2}\right)}{A} .
$$

By (5.11) it follows that for every $\delta^{\prime}>0$ there exist $A_{\delta^{\prime}}>0$ big enough and a compact set $K_{\delta^{\prime}} \subset \mathcal{C}_{\text {tem }}^{+}$ such that

$$
\inf _{\underline{\theta} \leq \theta \leq \bar{\theta}}\left(\nu_{T}^{*, l}(\theta)\right)\left(K_{\delta^{\prime}} \cap\left\{\left|R_{0}\left(u_{T_{1}+T_{2}}\right)\right|<A_{\delta^{\prime}}\right\}\right) \geq 1-\delta^{\prime} \quad \text { for all } T>1 .
$$

We obtain for $I_{2} \equiv \int_{\mathcal{C}_{\text {tem }}^{+}} \mathbb{P}_{f}\left(\mathbb{P}\left(R_{0}\left(w_{T_{1}+T_{2}}\right)>R_{0}\left(u_{T_{1}+T_{2}}\right) \mid \mathcal{F}_{T_{1}}^{u}\right) \leq \eta_{2}\left(\theta_{2}-\theta_{1}\right)\right)\left(\nu_{T}^{*, l}\left(\theta_{1}\right)\right)(d f)$ as in (3.37),

$$
\begin{array}{r}
I_{2} \geq \int_{K_{\delta^{\prime}}} \mathbb{P}_{f}\left(\sup _{a \in\left[-A_{\delta^{\prime}}, A_{\delta^{\prime}}\right]} \mathbb{E}\left[\mathbb{E}\left[1-e^{-2\left\langle v_{T_{1}},\left(u_{T_{2}}^{*, r}(\bar{\theta})\right)(\cdot-a)\right\rangle} \mid \sigma\left(\mathcal{F}^{u^{*, r}}, \mathcal{F}_{T_{1}}^{u}\right)\right] \mid \mathcal{F}_{T_{1}}^{u}\right] \leq \eta_{2}\left(\theta_{2}-\theta_{1}\right)\right) \\
\left(\nu_{T}^{*, l}\left(\theta_{1}\right)\right)(d f)-\delta^{\prime} .
\end{array}
$$


By symmetry, Corollaries 2.3 2.4 and the Markov inequality, for $T_{2}>0$ fixed we can choose $l>0$ big enough such that

$$
\mathbb{P}\left(\left|L_{0}\left(u_{T_{2}}^{*, r}(\bar{\theta})\right)\right| \geq l\right) \leq \delta^{\prime}
$$

Recall (3.4)-(3.5) and use monotonicity to conclude that for $T_{1}>0$ fixed, for all $r>0, T>1$,

$$
\mathbb{P}_{\nu_{T}^{*, l}\left(\theta_{1}\right)}\left(0 \vee R_{0}\left(v_{T_{1}}\right) \geq r\right) \leq \mathbb{P}_{\nu_{T}^{*, l}\left(\theta_{1}\right)}\left(0 \vee R_{0}\left(u_{T_{1}}\left(\theta_{2}\right)\right) \geq r\right) \leq \frac{C\left(\underline{\theta}, \bar{\theta}, T_{1}\right)}{r} .
$$

Thus, for $\delta^{\prime}>0$ fixed, we can pick $l, r>0$ big enough such that

$$
\begin{gathered}
I_{2} \geq \int_{K_{\delta^{\prime}}} \mathbb{P}_{f}\left(\sup _{a \in\left[-A_{\delta^{\prime}}, A_{\delta^{\prime}}\right]} \mathbb{E}\left[\mathbb{E}\left[1-e^{-2\left\langle v_{T_{1}},\left(\mathbb{1}_{[-l, r]} u_{T_{2}}^{*, r}(\bar{\theta})\right)(\cdot-a)\right\rangle} \mid \sigma\left(\mathcal{F}^{u^{*, r}}, \mathcal{F}_{T_{1}}^{u}\right)\right] \mid \mathcal{F}_{T_{1}}^{u}\right]\right. \\
\left.\leq \eta_{2}\left(\theta_{2}-\theta_{1}\right)\right)\left(\nu_{T}^{*, l}\left(\theta_{1}\right)\right)(d f)-3 \delta^{\prime} .
\end{gathered}
$$

Now reason as from above (3.33) to below (3.34), this time using (3.24) from the proof of the upper bound from Lemma 3.1, to obtain the claim.

Recall the following observation for the coupling from (3.5) from the beginning of the proof of Lemma 3.2. Conditional on $\mathcal{F}_{T_{1}}^{u}, v$ has law $Q_{v}^{0,\left(\theta_{2}-\theta_{1}\right) u, 2 u, 1}\left(\theta_{2}\right)$ on $\left[0, T_{1}\right]$ and conditional on $\mathcal{F}_{T_{1}}, w$ has law $Q_{w}^{u_{T_{1}}+v_{T_{1}}, 0,0,1}\left(\theta_{1}\right)$ on $\left[T_{1}, T_{1}+T_{2}\right]$. Finally, recall that $w=u+v$.

Lemma 3.6. Let $T_{1}, T_{2}>0$ be arbitrarily fixed, $\theta_{c}<\underline{\theta} \leq \theta_{1}<\theta_{2} \leq \bar{\theta}$. For all $\delta^{\prime}>0$ there exists $\eta_{3}=\eta_{3}\left(\delta^{\prime}, T_{1}, T_{2}, \underline{\theta}, \bar{\theta}\right)>0$ small enough such that

$$
\int_{\mathcal{C}_{\text {tem }}^{+}} \mathbb{E}_{f}\left[\mathbb{1}\left\{\mathbb{P}_{f}\left(\left\{R_{0}\left(w_{T_{1}+T_{2}}\right)-R_{0}\left(u_{T_{1}+T_{2}}\right) \geq \eta_{3}\right\} \mid \mathcal{F}_{T_{1}}^{u}\right) \geq \eta_{3}^{2} \mathbb{P}_{f}\left(\left\{R_{0}\left(w_{T_{1}+T_{2}}\right)>R_{0}\left(u_{T_{1}+T_{2}}\right)\right\} \mid \mathcal{F}_{T_{1}}^{u}\right)\right\}\right]\left(\nu_{T}^{*, l}\left(\theta_{1}\right)\right)(d f) \geq 1-6 \delta^{\prime}
$$

for all $T>1$.

Remark 3.7. Note that by (3.36), $\mathbb{P}\left(\left\{R_{0}\left(w_{T_{1}+T_{2}}\right)>R_{0}\left(u_{T_{1}+T_{2}}\right)\right\} \mid \mathcal{F}_{T_{1}}^{u}\right)>0$ almost surely under $\nu_{T}^{*, l}\left(\theta_{1}\right)$.

Proof. Let $X \geq 0$ be a random variable on some probability space $(\Omega, \mathcal{F}, \tilde{\mathbb{P}})$. Then (cf. [9, Proof of Lemma 3])

$$
\tilde{\mathbb{P}}\left(X>\frac{1}{2} \tilde{\mathbb{E}}[X]\right) \geq \frac{(\tilde{\mathbb{E}}[X])^{2}}{4 \tilde{\mathbb{E}}\left[X^{2}\right]} .
$$

In the coupling from above, let

$$
0 \leq X \equiv 0 \vee\left(R_{0}\left(w_{T_{1}+T_{2}}\right) \wedge 1\right)-0 \vee\left(R_{0}\left(u_{T_{1}+T_{2}}\right) \wedge 1\right) \leq 1 .
$$

In what follows, we make use of regular conditional distributions. For $\mathbb{P}\left(\left\{R_{0}\left(w_{T_{1}+T_{2}}\right)>R_{0}\left(u_{T_{1}+T_{2}}\right)\right\} \mid\right.$ $\left.\mathcal{F}_{T_{1}}^{u}\right)>0$, set

$$
\tilde{\mathbb{P}}(\{\cdot\})=\frac{\mathbb{P}\left(\{\cdot\} \cap\left\{R_{0}\left(w_{T_{1}+T_{2}}\right)>R_{0}\left(u_{T_{1}+T_{2}}\right)\right\} \mid \mathcal{F}_{T_{1}}^{u}\right)}{\mathbb{P}\left(\left\{R_{0}\left(w_{T_{1}+T_{2}}\right)>R_{0}\left(u_{T_{1}+T_{2}}\right)\right\} \mid \mathcal{F}_{T_{1}}^{u}\right)} .
$$

Now apply (3.50) to get for $\eta_{3} \leq \frac{1}{2} \tilde{\mathbb{E}}[X]$,

$$
\begin{aligned}
& \tilde{\mathbb{P}}\left(\left\{R_{0}\left(w_{T_{1}+T_{2}}\right)-R_{0}\left(u_{T_{1}+T_{2}}\right) \geq \eta_{3}\right\}\right) \\
& \geq \tilde{\mathbb{P}}\left(X \geq \eta_{3}\right) \geq \tilde{\mathbb{P}}\left(X>\frac{1}{2} \tilde{\mathbb{E}}[X]\right) \geq \frac{(\tilde{\mathbb{E}}[X])^{2}}{4 \tilde{\mathbb{E}}\left[X^{2}\right]} \geq \frac{(\tilde{\mathbb{E}}[X])^{2}}{4} \geq \eta_{3}^{2} .
\end{aligned}
$$


Therefore it suffices to show that there exists $\eta_{3}>0$ small enough such that

$$
\int_{\mathcal{C}_{\text {tem }}^{+}} \mathbb{E}_{f}\left[\mathbb{1}_{\left\{\eta_{3} \leq \tilde{\mathbb{E}}[X] / 2\right\}}\right]\left(\nu_{T}^{*, l}\left(\theta_{1}\right)\right)(d f) \geq 1-6 \delta^{\prime}
$$

By (3.25) and (3.37) we have for $\eta_{3}=\eta_{1} /\left(2 \eta_{2}\right)$,

$$
\begin{aligned}
& \int_{\mathcal{C}_{\text {tem }}^{+}} \mathbb{E}_{f}\left[\mathbb{1}_{\left.\left\{\eta_{3} \leq \tilde{\mathbb{E}}[X] / 2\right\}\right]}\right]\left(\nu_{T}^{*, l}\left(\theta_{1}\right)\right)(d f) \\
& =\int_{\mathcal{C}_{\text {tem }}^{+}} \mathbb{E}_{f}\left[\mathbb{1}\left\{\mathbb{E}\left[0 \vee\left(R_{0}\left(w_{T_{1}+T_{2}}\right) \wedge 1\right)-0 \vee\left(R_{0}\left(u_{T_{1}+T_{2}}\right) \wedge 1\right) \mid \mathcal{F}_{T_{1}}^{u}\right] \geq 2 \eta_{3} \mathbb{P}\left(\left\{R_{0}\left(w_{T_{1}+T_{2}}\right)>R_{0}\left(u_{T_{1}+T_{2}}\right)\right\} \mid \mathcal{F}_{T_{1}}^{u}\right)\right\}\right]\left(\nu_{T}^{*, l}\left(\theta_{1}\right)\right)(d f) \\
& \geq \int_{\mathcal{C}_{\text {tem }}^{+}} \mathbb{E}_{f}\left[\mathbb{1}\left\{\mathbb{E}\left[0 \vee\left(R_{0}\left(w_{T_{1}+T_{2}}\right) \wedge 1\right)-0 \vee\left(R_{0}\left(u_{T_{1}+T_{2}}\right) \wedge 1\right) \mid \mathcal{F}_{T_{1}}^{u}\right] \geq \eta_{1}\left(\theta_{2}-\theta_{1}\right)\right\}\right]\left(\nu_{T}^{*, l}\left(\theta_{1}\right)\right)(d f) \\
& -\int_{\mathcal{C}_{\text {tem }}^{+}} \mathbb{E}_{f}\left[\mathbb{1}\left\{\mathbb{P}\left(\left\{R_{0}\left(w_{T_{1}+T_{2}}\right)>R_{0}\left(u_{T_{1}+T_{2}}\right)\right\} \mid \mathcal{F}_{T_{1}}^{u}\right) \geq \frac{\eta_{1}\left(\theta_{2}-\theta_{1}\right)}{2 \eta_{3}}\right\}\right]\left(\nu_{T}^{*, l}\left(\theta_{1}\right)\right)(d f) \\
& \geq 1-2 \delta^{\prime}-4 \delta^{\prime} .
\end{aligned}
$$

Lemma 3.8. Let $\varphi \in \mathcal{C}_{\text {tem }}^{+}$with $L_{0}(\varphi) \in(0,1)$ be arbitrarily fixed. Further let $T_{1}, T_{2}>0$ be arbitrarily fixed and $\theta_{c}<\underline{\theta} \leq \theta_{1}<\theta_{2} \leq \bar{\theta}$. For all $\delta^{\prime}>0$ there exists $\eta_{4}=\eta_{4}\left(\varphi, \delta^{\prime}, T_{1}, T_{2}, \underline{\theta}, \bar{\theta}\right)>0$ small enough such that

$$
\int_{\mathcal{C}_{\text {tem }}^{+}} \mathbb{E}_{f}\left[\mathbb{1}\left\{\mathbb{P}_{f}\left(\left\{\left\langle v_{T_{1}+T_{2}}\left(\cdot+R_{0}\left(u_{T_{1}+T_{2}}\right)\right), \varphi(\cdot)\right\rangle \geq \eta_{4}\right\} \mid \mathcal{F}_{T_{1}}^{u}\right) \geq\left(1-e^{-2 \eta_{4}}\right)^{2} \mathbb{P}_{f}\left(\left\{R_{0}\left(w_{T_{1}+T_{2}}\right)>R_{0}\left(u_{T_{1}+T_{2}}\right)\right\} \mid \mathcal{F}_{T_{1}}^{u}\right)\right\}\right]
$$

$$
\left(\nu_{T}^{*, l}\left(\theta_{1}\right)\right)(d f) \geq 1-\delta^{\prime}
$$

for all $T>1$.

Proof. Let $\varphi \in \mathcal{C}_{\text {tem }}^{+}$with $L_{0}(\varphi) \in(0,1)$ and $\eta_{4}>0$ be arbitrarily fixed. Note that in what follows we condition first on $\mathcal{F}_{T_{1}+T_{2}}^{u}$ rather than on $\mathcal{F}_{T_{1}}^{u}$. Next rewrite

$$
\begin{aligned}
& \mathbb{P}\left(\left\{\left\langle v_{T_{1}+T_{2}}\left(\cdot+R_{0}\left(u_{T_{1}+T_{2}}\right)\right), \varphi(\cdot)\right\rangle \geq \eta_{4}\right\} \mid \mathcal{F}_{T_{1}+T_{2}}^{u}\right) \\
& =\mathbb{P}\left(\left\{1-e^{-2\left\langle v_{T_{1}+T_{2}}\left(\cdot+R_{0}\left(u_{T_{1}+T_{2}}\right)\right), \varphi(\cdot)\right\rangle} \geq 1-e^{-2 \eta_{4}}\right\} \mid \mathcal{F}_{T_{1}+T_{2}}^{u}\right) .
\end{aligned}
$$

Recall from (3.6) that by means of a monotonicity-coupling, $u_{T_{1}+t}(x)=u_{t}^{\left(u_{T_{1}}\left(\theta_{1}\right)\right)}\left(\theta_{1}\right)(x) \leq w_{T_{1}+t}(x)=$ $u_{t}^{\left(w_{T_{1}}\right)}\left(\theta_{1}\right)(x)=u_{T_{1}+t}(x)+v_{T_{1}+t}(x)$ for $0 \leq t \leq T_{2}$ with $v$ solving (cf. (5.21) $)$

$$
\frac{\partial v}{\partial t}=\Delta v+\left(\theta_{1}-v-2 u\right) v+\sqrt{v} \dot{W}_{2}, \quad v\left(T_{1}\right)=w_{T_{1}}-u_{T_{1}}, \quad T_{1} \leq t \leq T_{1}+T_{2} .
$$


By Corollary 5.1 we have

$$
\begin{aligned}
& \mathbb{E}\left[1-e^{-2\left\langle v_{T_{1}+T_{2}}\left(\cdot+R_{0}\left(u_{T_{1}+T_{2}}\right)\right), \varphi(\cdot)\right\rangle} \mid \mathcal{F}_{T_{1}+T_{2}}^{u}\right]=\mathbb{E}\left[1-e^{-2\left\langle v_{T_{1}+T_{2}}, \varphi\left(\cdot-R_{0}\left(u_{T_{1}+T_{2}}\right)\right)\right\rangle} \mid \mathcal{F}_{T_{1}+T_{2}}^{u}\right] \\
& =\mathbb{E}\left[1-e^{-2\left\langle v_{T_{1}}, z_{T_{1}+T_{2}}\right\rangle} \mid \mathcal{F}_{T_{1}+T_{2}}^{u}\right]
\end{aligned}
$$

where $z$ solves

$$
\frac{\partial z}{\partial t}=\Delta z+\left(\theta-z-2 u_{2 T_{1}+T_{2}-.}\right) z+\sqrt{z} \dot{W}_{3}, \quad z\left(T_{1}\right)=\varphi\left(\cdot-R_{0}\left(u_{T_{1}+T_{2}}\right)\right), \quad T_{1} \leq t \leq T_{1}+T_{2},
$$

where $W_{2}, W_{3}$ are independent white noises. That is, conditional on $\mathcal{F}_{T_{1}+T_{2}}^{u},\left(z_{t}\right)_{T_{1} \leq t \leq T_{1}+T_{2}}$ has law $\mathbb{P}(z) \equiv Q^{z\left(T_{1}\right), 0,2 u_{2 T_{1}+T_{2}-\cdot, 1}}$. By Theorem $\left.1.5 \mathrm{r}\right), \mathbb{P}(z)$ and $Q^{z\left(T_{1}\right), 0,0,0}$ are mutually absolutely continuous on $\mathcal{U}_{R, T}$ for $R, T>0$ arbitrarily fixed. The latter is the law of a superprocess with non-zero initial condition and thus is non-zero with positive probability at time $T_{1}+T_{2}$. Similarly, $v_{T_{1}}$ is non-zero with positive probability.

Now reason as in (3.28)-(3.34) with the following modifications. Use a $\theta$-coupling for $z$ to obtain (3.28). Then investigate

$$
\mathbb{E}\left[\mathbb{E}\left[1-e^{-2\left\langle v_{T_{1}}, z_{T_{1}+T_{2}}(\underline{\theta})\right\rangle} \mid \sigma\left(\mathcal{F}^{z}, \mathcal{F}_{T_{1}+T_{2}}^{u}\right)\right] \mathcal{F}_{T_{1}}^{u}\right]
$$

instead of the (outer) conditional expectation in (3.30). Only apply the lower bound of Lemma 3.1 in case $z_{T_{1}+T_{2}} \not \equiv 0$. This way we obtain a result in the spirit of (3.25). Here we do not require $z_{T_{1}+T_{2}}$ respectively $v_{T_{1}}$ to be non-zero a.s. as we do not have to multiply the (inner) conditional expectation from (3.61) with a front factor as in (3.30).

Note in particular, that the final statement is phrased in terms of conditioning on $\mathcal{F}_{T_{1}}^{u}$. Analogous reasoning to the proof of Lemma 3.6, using that $L_{0}(\varphi) \in(0,1)$ and thus $\left\langle v_{T_{1}+T_{2}}\left(\cdot+R_{0}\left(u_{T_{1}+T_{2}}\right)\right), \varphi(\cdot)\right\rangle=0$ if $R_{0}\left(w_{T_{1}+T_{2}}\right) \leq R_{0}\left(u_{T_{1}+T_{2}}\right)$, completes the proof.

Lemma 3.9. Let $\varphi \in \mathcal{C}_{\text {tem }}^{+}$with $L_{0}(\varphi) \in(0,1)$ and $T_{1}, T_{2}>0$ be arbitrarily fixed and $\theta_{c}<\underline{\theta} \leq \theta_{1}<\theta_{2} \leq \bar{\theta}$. For all $\delta^{\prime \prime}>0$ there exists $\eta_{5}=\eta_{5}\left(\varphi, \delta^{\prime \prime}, T_{1}, T_{2}, \underline{\theta}, \bar{\theta}\right)>0$ small enough such that

$$
\int_{\mathcal{C}_{\text {tem }}^{+}} \mathbb{P}_{f}\left(\mathbb{P}_{f}\left(\left\{\left\langle v_{T_{1}+T_{2}}\left(\cdot+R_{0}\left(u_{T_{1}+T_{2}}\right)\right), \varphi(\cdot)\right\rangle \geq \eta_{5}\right\} \mid \mathcal{F}_{T_{1}}^{u}\right) \geq \eta_{5}\left(\theta_{2}-\theta_{1}\right)\right)\left(\nu_{T}^{*, l}\left(\theta_{1}\right)\right)(d f) \geq 1-\delta^{\prime \prime}
$$

for all $T>1$.

Proof. By Corollary 3.4, with $\nu_{T}^{*, l}\left(\theta_{1}\right)(d f)$-measure of at least $1-2 \delta^{\prime}$,

$$
\mathbb{P}_{f}\left(\mathbb{P}_{f}\left(R_{0}\left(w_{T_{1}+T_{2}}\right)>R_{0}\left(u_{T_{1}+T_{2}}\right) \mid \mathcal{F}_{T_{1}}^{u}\right) \geq \eta_{1}\left(\theta_{2}-\theta_{1}\right)\right) \geq 1-2 \delta^{\prime}
$$

Hence, with $\nu_{T}^{*, l}\left(\theta_{1}\right)(d f) \mathbb{P}_{f}(d \omega)$-measure of at least $\left(1-2 \delta^{\prime}\right)^{2} \geq 1-4 \delta^{\prime}$,

$$
\mathbb{P}_{f}\left(R_{0}\left(w_{T_{1}+T_{2}}\right)>R_{0}\left(u_{T_{1}+T_{2}}\right) \mid \mathcal{F}_{T_{1}}^{u}\right)(\omega) \geq \eta_{1}\left(\theta_{2}-\theta_{1}\right) .
$$

Also, by Lemma 3.8, with $\nu_{T}^{*, l}\left(\theta_{1}\right)(d f)$-measure of at least $1-\delta^{\prime}$,

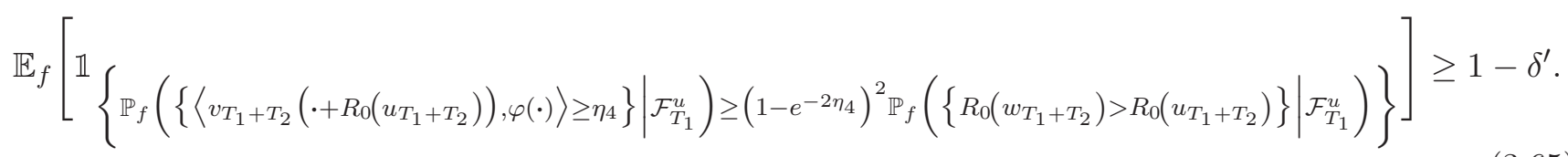


Hence, with $\nu_{T}^{*, l}\left(\theta_{1}\right)(d f) \mathbb{P}_{f}(d \omega)$-measure of at least $\left(1-\delta^{\prime}\right)^{2} \geq 1-2 \delta^{\prime}$,

$$
\begin{aligned}
& \mathbb{P}_{f}\left(\left\{\left\langle v_{T_{1}+T_{2}}\left(\cdot+R_{0}\left(u_{T_{1}+T_{2}}\right)\right), \varphi(\cdot)\right\rangle \geq \eta_{4}\right\} \mid \mathcal{F}_{T_{1}}^{u}\right)(\omega) \\
& \geq\left(1-e^{-2 \eta_{4}}\right)^{2} \mathbb{P}_{f}\left(\left\{R_{0}\left(w_{T_{1}+T_{2}}\right)>R_{0}\left(u_{T_{1}+T_{2}}\right)\right\} \mid \mathcal{F}_{T_{1}}^{u}\right)(\omega) .
\end{aligned}
$$

Together with (3.64) this yields that with $\nu_{T}^{*, l}\left(\theta_{1}\right)(d f) \mathbb{P}_{f}(d \omega)$-measure of at least $1-6 \delta^{\prime}$,

$$
\mathbb{P}_{f}\left(\left\{\left\langle v_{T_{1}+T_{2}}\left(\cdot+R_{0}\left(u_{T_{1}+T_{2}}\right)\right), \varphi(\cdot)\right\rangle \geq \eta_{4}\right\} \mid \mathcal{F}_{T_{1}}^{u}\right)(\omega) \geq\left(1-e^{-2 \eta_{4}}\right)^{2} \eta_{1}\left(\theta_{2}-\theta_{1}\right) .
$$

The claim now follows.

Proof of Proposition 2.17. Let $\varphi \in \mathcal{C}_{\text {tem }}^{+}$with $L_{0}(\varphi) \in(0,1), T_{1}, T_{2}, \xi>0$ and $\theta_{m-1}, \theta_{m}$ be arbitrarily fixed. For ease of notation, write $\theta_{1}, \theta_{2}$ instead of $\theta_{m-1}, \theta_{m}$ and set $\epsilon=\theta_{2}-\theta_{1}$. By Lemma 3.9 and the definition of $\nu_{T}^{*, l}\left(\theta_{1}\right)$ (cf. (5.8) $)$, for all $\delta^{\prime \prime}>0$ there exists $\eta_{6}>0$ small enough and $T_{0}>0$ big enough, all constants only dependent on $\varphi, \delta^{\prime \prime}, T_{1}, T_{2}, \underline{\theta}, \bar{\theta}$, such that

$$
\frac{1}{T} \int_{0}^{T} \mathbb{P}\left(\left\{\mathbb{P}_{u_{s}^{*, l}\left(\cdot+R_{0}(s)\right)}\left(\left\langle v_{T_{1}+T_{2}}\left(\cdot+R_{0}\left(u_{T_{1}+T_{2}}\right)\right), \varphi(\cdot)\right\rangle \geq \eta_{6}\right) \geq \eta_{6}\left(\theta_{2}-\theta_{1}\right)\right\}\right) d s \geq 1-\delta^{\prime \prime}
$$

for all $T \geq T_{0}$ and $\theta_{c}<\underline{\theta} \leq \theta_{1}<\theta_{2} \leq \bar{\theta}$. Hence, using Fubini-Tonelli's theorem, there exists a set $\Omega^{\prime}$ with $\mathbb{P}\left(\Omega^{\prime}\right) \geq 1-\delta^{\prime \prime}$, such that for all $\omega \in \Omega^{\prime}$,

$$
\left.\frac{1}{T} \int_{0}^{T} \mathbb{1}_{\left\{\mathbb{P}_{u_{s}^{*, l}\left(\cdot+R_{0}(s)\right)}\left(\left\langle v_{T_{1}+T_{2}}\left(\cdot+R_{0}\left(u_{T_{1}+T_{2}}\right)\right), \varphi(\cdot)\right\rangle \geq \eta_{6}\right) \geq \eta_{6} \epsilon\right.}\right\}^{d s \geq 1-\delta^{\prime \prime}}
$$

For all $\omega \in \Omega^{\prime}$, there exists

$$
s_{1}=s_{1}(\omega) \equiv \inf \left\{s \geq \xi: \mathbb{P}_{u_{s}^{*, l}\left(\cdot+R_{0}(s)\right)}\left(\left\langle v_{T_{1}+T_{2}}\left(\cdot+R_{0}\left(u_{T_{1}+T_{2}}\right)\right), \varphi(\cdot)\right\rangle \geq \eta_{6}\right) \geq \eta_{6} \epsilon\right\}
$$

satisfying $s_{1} \leq T / 2-\xi-T_{1}-T_{2}$. In case $\left\langle v_{T_{1}+T_{2}}^{\left(u_{s_{1}}^{*, l}\left(\cdot+R_{0}\left(s_{1}\right)\right)\right)}\left(\cdot+R_{0}\left(u_{T_{1}+T_{2}}^{\left(u_{s}^{*, l}\left(\cdot+R_{0}\left(s_{1}\right)\right)\right)}\right)\right), \varphi(\cdot)\right\rangle \geq \eta_{6}$, set $S=s_{1}+T_{1}+T_{2}$ and call this a success. In case of no success, by (3.69), there exists

$$
s_{2}=s_{2}(\omega) \equiv \inf \left\{s \geq s_{1}+T_{1}+T_{2}: \mathbb{P}_{u_{s}^{*, l}\left(\cdot+R_{0}(s)\right)}\left(\left\langle v_{T_{1}+T_{2}}\left(\cdot+R_{0}\left(u_{T_{1}+T_{2}}\right)\right), \varphi(\cdot)\right\rangle \geq \eta_{6}\right) \geq \eta_{6} \epsilon\right\}
$$

satisfying $s_{2} \leq T / 2-\xi-T_{1}-T_{2}$. Continue as above with $s_{2}$ instead of $s_{1}$. By choosing $C>0$ small enough, we can repeat this procedure $\lceil C T\rceil$ times. If the above procedure fails, which can only happen if $\omega \notin \Omega^{\prime}$ or $\omega \in \Omega^{\prime}$ but there was no success in $\lceil C T\rceil$ trials, set $S=T / 2-\xi$.

Note that for $\eta, \delta^{\prime \prime} \in(0,1)$ arbitrarily fixed, $\max _{x \in\left[0, \delta^{\prime \prime}\right]}(x+(1-x) \eta)=\delta^{\prime \prime}+\left(1-\delta^{\prime \prime}\right) \eta$. As a result, using the strong Markov property of the family of laws $\mathbb{P}_{f}, f \in \mathcal{C}_{\text {tem }}^{+}$, we get

$$
\mathbb{P}\left(\nexists S: \xi \leq S \leq T / 2-\xi:\left\langle\Delta_{S}^{*, l}\left(\theta_{1}, \theta_{2}\right), \varphi\right\rangle \geq \eta_{6}\right) \leq \delta^{\prime \prime}+\left(1-\delta^{\prime \prime}\right)\left(1-\eta_{6} \epsilon\right)^{\lceil C T\rceil} .
$$

Recall from (2.43) that $\epsilon=\delta / T$ to conclude that

$$
\mathbb{P}\left(\exists S: \xi \leq S \leq T / 2-\xi:\left\langle\Delta_{S}^{*, l}\left(\theta_{1}, \theta_{2}\right), \varphi\right\rangle \geq \eta_{6}\right) \geq\left(1-\delta^{\prime \prime}\right)\left(1-\left(1-\eta_{6} \delta / T\right)^{\lceil C T\rceil}\right) .
$$

For $T \rightarrow \infty$ this bound approaches $\left(1-\delta^{\prime \prime}\right)\left(1-\exp \left(-C \eta_{6} \delta\right)\right)>0$. This completes the proof of the claim. 


\section{The speed of the right marker}

Note the construction of travelling wave solutions from Theorem 5.7 respectively Remark 5.8 of the appendix. Let $\nu_{T}^{*, l} \in \mathcal{P}\left(\mathcal{C}_{\text {tem }}^{+}\right)$be given as in (5.8) and denote any arbitrary subsequential limit of the tight set $\left\{\nu_{T}^{*, l}: T \geq 1\right\}$ by $\nu=\nu^{*, l}$ in what follows. This limit yields a travelling wave solution to (1.1). By Proposition [5.6, $\nu^{*, l}\left(\left\{f: R_{0}(f)=0\right\}\right)=1$ and $\mathbb{P}_{\nu^{*}, l}(u(t) \not \equiv 0)=1$ for all $t \geq 0$. Denote with $\nu^{\left(u_{0}\right)}$ any subsequential limit that is obtained as in Remark 5.8 for $u_{0} \in \mathcal{H}$ with analogous properties.

Recall from [19, Proposition 4.1] that for $\theta>\theta_{c}$ and $\left(u_{t}^{(\nu)}\right)_{t \geq 0}$ a travelling wave solution to (1.1),

$$
R_{0}\left(u_{t}^{(\nu)}\right) / t \rightarrow A^{(\nu)}=A^{(\nu)}(\omega) \in\left[0,2 \theta^{1 / 2}\right] \text { almost surely as } t \rightarrow \infty
$$

holds. This convergence also holds in $\mathcal{L}^{1}$ if we replace $R_{0}(u(t))$ by $0 \vee R_{0}(u(t))$ as we see below.

In this section we show that the limiting speed of the dominating right marker $R_{0}\left(u_{t}^{*, l}\right)$ and that of any travelling wave solution $\nu^{*, l}$ coincide. Moreover, the speed is deterministic, namely it equals $B=B(\theta)$ from Lemma 2.7. We extend this result to right front markers of solutions to (1.1) with initial conditions $\psi$ satisfying $\psi \in \mathcal{H}^{R}$, where the convergence is now in probability and $\mathcal{L}^{1}$. For the right front marker of a corresponding travelling wave solution we obtain almost sure convergence to $A^{\left(\nu^{(\psi)}\right)}=B$.

Lemma 4.1. Let $\theta>\theta_{c}$. Then, for any $\left(u_{t}^{(\nu)}\right)_{t \geq 0}$ a travelling wave solution to (1.1),

$$
\left(0 \vee R_{0}\left(u_{t}^{(\nu)}\right)\right) / t \rightarrow A^{(\nu)}=A^{(\nu)}(\omega) \in\left[0,2 \theta^{1 / 2}\right] \quad \text { as } t \rightarrow \infty \text { in } \mathcal{L}^{1} .
$$

Moreover, $\mathbb{E}\left[A^{(\nu)}\right] \leq B$.

Proof. By (4.1), for all $N \in \mathbb{N},\left(\left(0 \vee R_{0}\left(u_{t}^{(\nu)}\right)\right) \wedge(N t)\right) / t \rightarrow A^{(\nu)} \wedge N$ almost surely for $t \rightarrow \infty$. By dominated convergence,

$$
\left(\left(0 \vee R_{0}\left(u_{t}^{(\nu)}\right)\right) \wedge(N t)\right) / t \rightarrow A^{(\nu)} \wedge N \quad \text { as } t \rightarrow \infty \text { in } \mathcal{L}^{1}
$$

By [14, (2.33)] and Lemma 2.13, for $m>0$ arbitrary,

$$
\mathbb{P}\left(R_{0}\left(u_{t}^{(\nu)}\right) / t \geq m\right) \leq C m^{-2}
$$

uniformly in $t \geq 1$. In combination with (4.1) this gives $\mathbb{P}\left(A^{(\nu)} \geq 2 m\right) \leq C m^{-2}$. Thus $A^{(\nu)} \in \mathcal{L}^{1}$. For $N \in \mathbb{N}$ arbitrary, we get

$$
\frac{\mathbb{E}\left[\left(0 \vee R_{0}\left(u_{t}^{(\nu)}\right)\right) \wedge(N t)\right]}{t} \leq \frac{\mathbb{E}\left[0 \vee R_{0}\left(u_{t}^{(\nu)}\right)\right]}{t} \leq \frac{\mathbb{E}\left[\left(0 \vee R_{0}\left(u_{t}^{(\nu)}\right)\right) \wedge(N t)\right]}{t}+\int_{N}^{\infty} C m^{-2} d m
$$

and similarly, $\left|\mathbb{E}\left[A^{(\nu)} \wedge N\right]-\mathbb{E}\left[A^{(\nu)}\right]\right| \leq 2 C / N$. Hence,

$$
\lim _{t \rightarrow \infty} \mathbb{E}\left[\left|\frac{0 \vee R_{0}\left(u_{t}^{(\nu)}\right)}{t}-A^{(\nu)}\right|\right] \leq \lim _{t \rightarrow \infty} \mathbb{E}\left[\left|\frac{\left(0 \vee R_{0}\left(u_{t}^{(\nu)}\right)\right) \wedge(N t)}{t}-A^{(\nu)} \wedge N\right|\right]+C / N+2 C / N=3 C / N
$$

and the first claim follows after taking $N \rightarrow \infty$.

Moreover, for all $N \in \mathbb{N}$, using once more [14, (2.33)] and the $\mathcal{L}^{1}$-convergence of the first claim,

$$
\begin{aligned}
\mathbb{E}\left[A^{(\nu)}\right] \leq \mathbb{E}\left[A^{(\nu)} \wedge N\right]+2 C / N & =\lim _{T \rightarrow \infty} \frac{\mathbb{E}\left[\left(0 \vee R_{0}\left(u_{T}^{(\nu)}\right)\right) \wedge(N T)\right]}{T}+2 C / N \\
& \leq \lim _{T \rightarrow \infty} \frac{\mathbb{E}\left[0 \vee R_{0}\left(u_{T}^{*, l}\right)\right]}{T}+2 C / N \leq B+2 C / N .
\end{aligned}
$$

Take $N \rightarrow \infty$ to conclude that $\mathbb{E}\left[A^{(\nu)}\right] \leq B$. 
Recall from Lemma 2.7 and Corollary 2.11 that

$$
\lim _{T \rightarrow \infty} \frac{\mathbb{E}\left[R_{0}\left(u_{T}^{*, l}\right)\right]}{T}=\inf _{T \geq 1} \frac{\mathbb{E}\left[R_{0}\left(u_{T}^{*, l}\right)\right]}{T} \equiv B \in(0, \infty) .
$$

Then

$$
B=\lim _{T \rightarrow \infty} \frac{\mathbb{E}\left[0 \vee R_{0}\left(u_{T}^{*, l}\right)\right]}{T}
$$

holds as well. Indeed, let $\left(u_{t}^{(\nu)}\right)_{t \geq 0}$ be an arbitrary travelling wave solution with $R_{0}(\nu)=0$ almost surely. By Corollary [2.2 and [14, (2.33)], (4.1), for $M \in \mathbb{N}$ arbitrary,

$$
\begin{aligned}
\lim _{T \rightarrow \infty} \mathbb{E}\left[0 \vee\left(-R_{0}\left(u_{T}^{*, l}\right)\right)\right] / T & \leq \lim _{T \rightarrow \infty} \mathbb{E}\left[\left(0 \vee\left(-R_{0}\left(u_{T}^{*, l}\right)\right)\right) \wedge(M T)\right] / T+C_{1} e^{-C_{2} M} \\
& \leq \lim _{T \rightarrow \infty} \mathbb{E}\left[\left(0 \vee\left(-R_{0}\left(u_{T}^{(\nu)}\right)\right)\right) \wedge(M T)\right] / T+C_{1} e^{-C_{2} M} \\
& =\mathbb{E}\left[0 \vee\left(-A^{(\nu)} \wedge M\right)\right]+C_{1} e^{-C_{2} M}=C_{1} e^{-C_{2} M} .
\end{aligned}
$$

Take $M \rightarrow \infty$ and the claim follows.

Lemma 4.2. Let $\theta>\theta_{c}$. With the notations from above, $\mathbb{E}\left[A^{\left(\nu^{*, l}\right)}\right]=B$ holds true. Moreover, if $\psi \in \mathcal{H}^{R}$ satisfies $R_{0}\left(u_{T}^{(\psi)}\right) / T \rightarrow B$ for $T \rightarrow \infty$ in $\mathcal{L}^{1}$, then $\mathbb{E}\left[A^{\left(\nu^{(\psi)}\right)}\right]=B$ holds as well.

Remark 4.3. Note that we will show at the end of this section (Corollary 4.7) that every $\psi \in \mathcal{H}$ indeed satisfies the above assumption.

Proof. Let $\nu=\nu^{*, l}$ or $\nu=\nu^{(\psi)}$ with $\psi$ as above. By Lemma 4.1, $\mathbb{E}\left[A^{(\nu)}\right] \leq B$. It remains to show that $\mathbb{E}\left[A^{(\nu)}\right] \geq B$. In what follows, we provide a proof in case $\nu=\nu^{*, l}$. The proof in case $\nu=\nu^{(\psi)}$ is analogous except for the changes indicated below.

Fix $T \geq 1$ arbitrary. Review the definitions and comments in [14, (5.18)-(5.20)]. Note in particular that for fixed $N \in \mathbb{N}$ and $m_{0}>0, R_{m_{0}}^{N}$ is a continuous function on $\mathcal{C}_{\text {tem }}^{+}$with $\left|R_{m_{0}}^{N}(f)\right| \leq N$ and $R^{m_{0}, N}(f) \leq R_{m_{0}}^{N}(f) \leq R_{0}(f)$ on $\left\{R_{0}(f) \geq-N\right\}$ and $R_{m_{0}}^{N}(f)=-N$ on $\left\{R_{0}(f)<-N\right\}$. Hence, for $m_{0}=m_{0}(T), N=N(T)$, by Lemma 4.1.

$$
\mathbb{E}\left[A^{\left(\nu^{*, l}\right)}\right]=\lim _{T \rightarrow \infty} \mathbb{E}_{\nu^{*, l}}\left[0 \vee R_{0}\left(u_{T}\right)\right] / T \geq \lim _{T \rightarrow \infty} \mathbb{E}_{\nu^{*, l}}\left[0 \vee R_{m_{0}}^{N}\left(u_{T}\right)\right] / T
$$

By the definition of tightness respectively weak convergence and the continuity of $f \mapsto \mathbb{P}_{f}$, (5.8) yields for $\nu_{T_{n}}^{*, l} \Rightarrow \nu^{*, l}(n \rightarrow \infty)$,

$$
\begin{aligned}
& \mathbb{E}_{\nu^{*, l}}\left[0 \vee R_{m_{0}}^{N}\left(u_{T}\right)\right]=\lim _{n \rightarrow \infty} \frac{1}{T_{n}} \int_{0}^{T_{n}} \mathbb{E}\left[0 \vee R_{m_{0}}^{N}\left(u_{s+T}^{*, l}\left(\cdot+R_{0}\left(u_{s}^{*, l}\right)\right)\right)\right] d s \\
& \geq \liminf _{n \rightarrow \infty}\left\{\frac{1}{T_{n}} \int_{0}^{T_{n}} \mathbb{E}\left[R_{0}\left(u_{s+T}^{*, l}\left(\cdot+R_{0}\left(u_{s}^{*, l}\right)\right)\right)\right] d s\right. \\
& \left.\quad-\frac{1}{T_{n}} \int_{0}^{T_{n}} \mathbb{E}\left[0 \vee\left\{R_{0}\left(u_{s+T}^{*, l}\left(\cdot+R_{0}\left(u_{s}^{*, l}\right)\right)\right)-R_{m_{0}}^{N}\left(u_{s+T}^{*, l}\left(\cdot+R_{0}\left(u_{s}^{*, l}\right)\right)\right)\right\}\right] d s\right\} \\
& \geq \liminf _{n \rightarrow \infty}\left\{\frac{1}{T_{n}} \int_{0}^{T_{n}} \mathbb{E}\left[R_{0}\left(u_{s+T}^{*, l}\left(\cdot+R_{0}\left(u_{s}^{*, l}\right)\right)\right)\right] d s\right\} \\
& \quad-\limsup _{n \rightarrow \infty}\left\{\frac{1}{T_{n}} \int_{0}^{T_{n}} \mathbb{E}\left[0 \vee\left\{R_{0}\left(u_{s+T}^{*, l}\left(\cdot+R_{0}\left(u_{s}^{*, l}\right)\right)\right)-R_{m_{0}}^{N}\left(u_{s+T}^{*, l}\left(\cdot+R_{0}\left(u_{s}^{*, l}\right)\right)\right)\right\}\right] d s\right\} \\
& \equiv I_{1}(T)-E_{1}\left(T, m_{0}(T), N(T)\right)=I_{1}(T)-E_{1}(T) .
\end{aligned}
$$


We obtain for $I_{1}$, using Corollaries 2.3 2.4, that

$$
\begin{aligned}
I_{1}(T) & =\liminf _{n \rightarrow \infty} \frac{1}{T_{n}} \int_{0}^{T_{n}} \mathbb{E}\left[R_{0}\left(u_{s+T}^{*, l}\right)-R_{0}\left(u_{s}^{*, l}\right)\right] d s \\
& =\liminf _{n \rightarrow \infty} \frac{1}{T_{n}}\left(\int_{T_{n}}^{T_{n}+T} \mathbb{E}\left[R_{0}\left(u_{s}^{*, l}\right)\right] d s-\int_{0}^{T} \mathbb{E}\left[R_{0}\left(u_{s}^{*, l}\right)\right] d s\right)=T B
\end{aligned}
$$

by (4.8) respectively the assumption $R_{0}\left(u_{T}^{(\psi)}\right) / T \rightarrow B$ for $T \rightarrow \infty$ in $\mathcal{L}^{1}$ for $\psi \in \mathcal{H}^{R}$. It therefore remains to show that $\lim \sup _{T \rightarrow \infty} E_{1}(T) / T=0$.

For $\epsilon>0$ arbitrarily fixed, recall the definition of $R^{m_{0}, N}(f)$ from [14, (5.18)]. Also recall from above that $\left|R_{m_{0}}^{N}(f)\right| \leq N$ and $R^{m_{0}, N}(f) \leq R_{m_{0}}^{N}(f) \leq R_{0}(f)$ on $\left\{R_{0}(f) \geq-N\right\}$ and $R_{m_{0}}^{N}(f)=-N$ on $\left\{R_{0}(f)<-N\right\}$ to obtain that

$$
\begin{aligned}
E_{1}(T) \leq & \limsup _{n \rightarrow \infty} \frac{1}{T_{n}} \int_{0}^{T_{n}} \mathbb{E}\left[2 R_{0}\left(u_{s+T}^{*, l}\left(\cdot+R_{0}\left(u_{s}^{*, l}\right)\right)\right) \mathbb{1}_{\left.\left\{R_{0}\left(u_{s+T}^{*, l}\left(\cdot+R_{0}\left(u_{s}^{*, l}\right)\right)\right)>N\right\}\right] d s}\right. \\
& +\limsup _{n \rightarrow \infty} \frac{1}{T_{n}} \int_{0}^{T_{n}} \mathbb{E}\left[2 N \mathbb{1}_{\left\{\left\langle u_{s+T}^{*, l}\left(\cdot+R_{0}\left(u_{s+T}^{*, l}\right)\right), \mathbb{1}_{[-\epsilon T, \infty)}\right\rangle<m_{0}\right\}}\right] d s+\epsilon T \\
= & 2 \limsup _{n \rightarrow \infty} \mathbb{E}_{\nu_{T_{n}}^{*, l}}\left[R_{0}\left(u_{T}\right) \mathbb{1}_{\left\{R_{0}\left(u_{T}\right)>N\right\}}\right]+2 N \limsup _{n \rightarrow \infty} \mathbb{P}_{\nu_{T_{n}}^{*, l}}\left(\left\langle u_{T}\left(\cdot+R_{0}(T)\right), \mathbb{1}_{[-\epsilon T, \infty)}\right\rangle<m_{0}\right)+\epsilon T .
\end{aligned}
$$

By [14, (2.33)] and Lemma 2.13 we choose $N>C T / \epsilon$ such that

$$
\mathbb{E}_{\nu_{T_{n}}^{*, l}}\left[R_{0}\left(u_{T}\right) \mathbb{1}_{\left\{R_{0}\left(u_{T}\right)>N\right\}}\right] \leq \mathbb{E}\left[R_{0}\left(u_{T}^{*, l}\right) \mathbb{1}_{\left\{R_{0}\left(u_{T}^{*, l}\right)>N\right\}}\right] \leq N^{-1} \mathbb{E}\left[\left(0 \vee R_{0}\left(u_{T}^{*, l}\right)\right)^{2}\right] \leq N^{-1} C T^{2}<\epsilon T
$$

for all $n \in \mathbb{N}$. Finally, by Lemma 5.4 with $a=\epsilon T / 2$, we choose $b=b(T)$ and $\tilde{m}=m_{0}(T)$ small enough such that

$$
\mathbb{P}_{\nu_{T_{n}}^{*, l}}\left(\left\langle u_{T}\left(\cdot+R_{0}(T)\right), \mathbb{1}_{[-\epsilon T, \infty)}\right\rangle<m_{0}\right) \leq \frac{\epsilon T}{2 N(T)}
$$

for all $n \in \mathbb{N}$. Thus, $E_{1}(T) / T \leq 4 \epsilon$ for all $T \geq 1$ and the claim follows after taking $\epsilon \downarrow 0^{+}$.

Proposition 4.4. Let $\theta>\theta_{c}$. Then $A^{\left(\nu^{*, l}\right)} \equiv \mathbb{E}\left[A^{\left(\nu^{*, l}\right)}\right]=B$ almost surely and

$$
R_{0}\left(u_{T}^{*, l}\right) / T \rightarrow B \text { almost surely as } T \rightarrow \infty .
$$

In particular, $A^{(\nu)} \leq B$ almost surely for all $A^{(\nu)}$ as in (4.1).

Proof. The last claim follows immediately from [14, (2.33)].

Fix $T_{0}>0$. By [14, (2.33)] and as $\nu^{*, l}\left(\left\{f: R_{0}(f)=0\right\}\right)=1$ by Proposition 5.6, there exists a coupling such that

$$
R_{0}\left(u_{T_{0}+t}^{\left(\nu^{*, l}\right)}\right) \leq R_{0}\left(u_{T_{0}+t}^{*, l}\right) \quad \text { for all } t \geq 0 \text { almost surely. }
$$

By Corollaries 2.3 2.4, $\mathbb{E}\left[\left|R_{0}\left(u_{T_{0}}^{*, l}\right)\right|\right] \leq C\left(T_{0}\right)$ and thus, using Lemma 4.1 and (4.1),

$$
A^{\left(\nu^{*, l}\right)}=\lim _{T \rightarrow \infty} \frac{0 \vee R_{0}\left(u_{T}^{\left(\nu^{*, l}\right)}\right)}{T}=\lim _{T \rightarrow \infty} \frac{R_{0}\left(u_{T}^{\left(\nu^{*, l}\right)}\right)}{T} \leq \liminf _{T \rightarrow \infty} \frac{R_{0}\left(u_{T}^{*, l}\right)}{T} \quad \text { a.s. },
$$

where the left equality also holds in $\mathcal{L}^{1}$. 
Note that by reasoning as in the proof of [19, Proposition 4.1a)], for $T>0$ fixed, once we bound $\limsup _{n \rightarrow \infty} R_{0}\left(u_{T_{0}+n T}^{*, l}\right) / n T$, the same bound holds for $\lim _{\sup _{t \rightarrow \infty}} R_{0}\left(u_{t}^{*, l}\right) / t$ almost surely. We therefore fix $T>0$ and rewrite

$$
\begin{aligned}
& \frac{1}{n T} R_{0}\left(u_{T_{0}+n T}^{*, l}\right)-\frac{1}{n T} R_{0}\left(u_{T_{0}}^{*, l}\right) \\
& =\frac{1}{n T} \sum_{i=1}^{n}\left(R_{0}\left(u_{T_{0}+i T}^{*, l}\right)-R_{0}\left(u_{T_{0}+(i-1) T}^{*, l}\right)\right)=\frac{1}{n T} \sum_{i=1}^{n} R_{0}\left(u_{T}^{\left(u_{T_{0}+(i-1) T}^{*,}\left(\cdot+R_{0}\left(u_{T_{0}+(i-1) T}^{*, l}\right)\right)\right)}\right) .
\end{aligned}
$$

Fix $i \in \mathbb{N}$. By (1.13), there exists a coupling such that

$$
R_{0}\left(u_{T}^{\left(u_{T_{0}+(i-1) T}^{*, l}\left(\cdot+R_{0}\left(u_{T_{0}+(i-1) T}^{*, l}\right)\right)\right)}\right) \leq R_{0}\left(u_{T}^{*, l}(i)\right) \text { almost surely, }
$$

where $\mathcal{L}\left(u_{T}^{*, l}(i)\right)=\mathcal{L}\left(u_{T}^{*, l}\right)$ for all $i \in \mathbb{N}$. By construction, the $\mathcal{L}\left(u_{T}^{*, l}(i)\right), i \in \mathbb{N}$ are independent. Indeed, we show this by induction. Let $u_{T_{0}+(i-1) T}^{*, l}$ be given. Then $\zeta_{1} \geq u_{T_{0}+(i-1) T}^{*, l}\left(\cdot+R_{0}\left(u_{T_{0}+(i-1) T}^{*, l}\right)\right)$ is chosen in the construction. Nevertheless, as $\zeta_{N}(x) \uparrow \infty$ for $x<0$ and $\zeta_{N}(x)=0$ for $x \geq 0$, the law of $u_{T}^{*, l}(i)$ conditional on $u_{T_{0}+(i-1) T}^{*, l}$ remains $\mathcal{L}\left(u_{T}^{*, l}\right)$. Thus

$$
\frac{1}{n T} R_{0}\left(u_{T_{0}+n T}^{*, l}\right)-\frac{1}{n T} R_{0}\left(u_{T_{0}}^{*, l}\right) \leq \frac{1}{n T} \sum_{i=1}^{n} R_{0}\left(u_{T}^{*, l}(i)\right),
$$

where $\left(R_{0}\left(u_{T}^{*, l}(i)\right)\right)_{i \in \mathbb{N}}$ is an i.i.d. sequence of real valued random variables with $R_{0}\left(u_{T}^{*, l}(1)\right) \stackrel{\mathcal{D}}{=} R_{0}\left(u_{T}^{*, l}\right)$. By Corollaries 2.3 2.4, $R_{0}\left(u_{T}^{*, l}\right) \in \mathcal{L}^{1}$.

By the ergodic theorem (cf. for instance Klenke [13, Theorems 20.14, 20.16 and Example 20.12]),

$$
\frac{1}{n T} \sum_{i=1}^{n} R_{0}\left(u_{T}^{*, l}(i)\right) \rightarrow \mathbb{E}\left[R_{0}\left(u_{T}^{*, l}\right)\right] / T \quad \text { almost surely and in } \mathcal{L}^{1} \text { for } n \rightarrow \infty .
$$

As a result,

$$
\limsup _{n \rightarrow \infty} \frac{R_{0}\left(u_{T_{0}+n T}^{*, l}\right)}{n T} \leq \limsup _{n \rightarrow \infty} \frac{R_{0}\left(u_{T_{0}}^{*, l}\right)}{n T}+\frac{\mathbb{E}\left[R_{0}\left(u_{T}^{*, l}\right)\right]}{T}=\frac{\mathbb{E}\left[R_{0}\left(u_{T}^{*, l}\right)\right]}{T}
$$

for all $T>0$. Take $T \rightarrow \infty$ to conclude that

$$
A^{\left(\nu^{*, l}\right)} \leq \liminf _{T \rightarrow \infty} \frac{R_{0}\left(u_{T}^{*, l}\right)}{T} \leq \limsup _{T \rightarrow \infty} \frac{R_{0}\left(u_{T}^{*, l}\right)}{T} \leq \limsup _{T \rightarrow \infty} \frac{\mathbb{E}\left[R_{0}\left(u_{T}^{*, l}\right)\right]}{T}=B=\mathbb{E}\left[A^{\left(\nu^{*, l}\right)}\right]
$$

and therefore $\lim _{T \rightarrow \infty} R_{0}\left(u_{T}^{*, l}\right) / T=B$ almost surely.

Corollary 4.5. Let $\theta>\theta_{c}$. Then

$$
R_{0}\left(u_{T}^{\left(\nu^{*, l}\right)}\right) / T \rightarrow B \text { almost surely as } T \rightarrow \infty
$$

and

$$
R_{0}\left(u_{T}^{*, l}\right) / T \rightarrow B \text { in } \mathcal{L}^{1} \text { as } T \rightarrow \infty .
$$

Proof. The first claim follows from (4.1) and Proposition 4.4.

By Proposition 4.4 we have $\left(R_{0}\left(u_{T}^{*, l}\right) / T \wedge N\right) \vee(-N) \rightarrow B$ for $T \rightarrow \infty$ almost surely for all $N \in$ $\mathbb{N}, N>B$ fixed. As these random variables are bounded, dominated convergence implies convergence in $\mathcal{L}^{1}$. We conclude that for all $N \in \mathbb{N}, N>B$,

$$
\limsup _{T \rightarrow \infty} \mathbb{E}\left[\left|\frac{R_{0}\left(u_{T}^{*, l}\right)}{T}-B\right|\right] \leq 2 \limsup _{T \rightarrow \infty} \mathbb{E}\left[\left|\frac{R_{0}\left(u_{T}^{*, l}\right)}{T}\right| \mathbb{1}_{\left\{\left|R_{0}\left(u_{T}^{*, l}\right)\right|>N T\right\}}\right] .
$$

The second claim now follows from the bounds on the positive part from Lemma 2.13 and on the negative part from (2.10) for $N \rightarrow \infty$. 
Finally, we consider initial conditions $\psi \in \mathcal{H}$ with $\mathcal{H}$ as in (1.15).

Lemma 4.6. For initial conditions $\psi \in \mathcal{H}^{R}, R_{0}\left(u_{T}^{(\psi)}\right) / T \stackrel{\mathcal{D}}{\rightarrow} B$ as $T \rightarrow \infty$.

Proof. Without loss of generality we assume that $\psi(x)=\epsilon H_{0}\left(x-x_{0}\right)$ for some $x_{0} \in \mathbb{R}, \epsilon>0$. Indeed, by definition of $\mathcal{H}^{R}$, for every $\psi \in \mathcal{H}$ there exist $x_{0} \in \mathbb{R}, \epsilon>0$ such that $\psi \geq \epsilon H_{0}\left(\cdot-x_{0}\right)$ and $R_{0}(\psi) \in$ $\mathbb{R}$. Reason as in [14, Remark 2.8(ii)] to construct a coupling such that for $T_{0}>0$ arbitrarily fixed, $u^{\left(\epsilon H_{0}\left(\cdot-x_{0}\right)\right)}(t, x) \leq u^{(\psi)}(t, x) \leq u^{*, l}\left(t, x-R_{0}(\psi)\right)$ for all $t \geq T_{0}, x \in \mathbb{R}$ almost surely. Then

$$
R_{0}\left(u_{t}^{\left(\epsilon H_{0}\left(\cdot-x_{0}\right)\right)}\right) / t \leq R_{0}\left(u_{t}^{(\psi)}\right) / t \leq\left(R_{0}\left(u_{t}^{*, l}\right)+R_{0}(\psi)\right) / t \text { for all } t \geq T_{0} \text { almost surely }
$$

and by Proposition 4.4,

$$
\lim _{t \rightarrow \infty}\left(R_{0}\left(u_{t}^{*, l}\right)+R_{0}(\psi)\right) / t=B \text { almost surely. }
$$

Hence, $R_{0}\left(u_{t}^{\left(\epsilon H_{0}\left(\cdot-x_{0}\right)\right)}\right) / t \stackrel{\mathcal{D}}{\rightarrow} B$ implies $R_{0}\left(u_{t}^{(\psi)}\right) / t \stackrel{\mathcal{D}}{\rightarrow} B$. By the shift invariance of the dynamics, assume further that $x_{0}=1$.

Let $c \in \mathbb{R}$ be arbitrary. For $c>B, \lim _{T \rightarrow \infty} \mathbb{P}\left(R_{0}\left(u_{T}^{(\psi)}\right) \geq c T\right)=0$ follows from (4.29)-(4.30). Note that $\psi(x)=\epsilon H_{0}(x-1) \geq \epsilon \mathbb{1}_{(-\infty, 0]}(x)$ for all $x \in \mathbb{R}$. By (1.11), symmetry and by the shift invariance of the dynamics, for all $T>0$,

$$
\begin{aligned}
\mathbb{P}\left(R_{0}\left(u_{T}^{(\psi)}\right)>c T\right) & =1-\mathbb{E}\left[e^{-2\left\langle\psi, u_{T}^{*, r}(\cdot-c T)\right\rangle}\right] \geq 1-\mathbb{E}\left[e^{-2 \epsilon\left\langle\mathbb{1}_{(-\infty, 0]}, u_{T}^{*, r}(\cdot-c T)\right\rangle}\right] \\
& \geq 1-\mathbb{P}\left(\left\langle\mathbb{1}_{(-\infty, 0]}, u_{T}^{*, r}(\cdot-c T)\right\rangle<N\right)-e^{-2 \epsilon N}
\end{aligned}
$$

for all $N \in \mathbb{N}$. Suppose $c<B$. Let $\delta>0$ and choose $N$ big enough such that $e^{-2 \epsilon N}<\delta$. As we will show below, for $N$ fixed,

$$
\lim _{T \rightarrow \infty} \mathbb{P}\left(\left\langle\mathbb{1}_{(-\infty, 0]}, u_{T}^{*, r}(\cdot-c T)\right\rangle<N\right)=0
$$

and therefore $\mathbb{P}\left(R_{0}\left(u_{T}^{(\psi)}\right)>c T\right) \geq 1-2 \delta$ for all $T$ big enough. Then $\lim _{T \rightarrow \infty} \mathbb{P}\left(R_{0}\left(u_{T}^{(\psi)}\right) \geq c T\right)=$ $\mathbb{1}_{(-\infty, B)}(c)$ for $c \neq B$ arbitrary follows.

It thus remains to show (4.32) for $0<c<B$ and $N$ arbitrarily fixed. Let $\Delta=(B-c) / 2,0<\Delta<B$. By symmetry, $L_{0}\left(u_{T}^{*, r}\right) / T \rightarrow-B$ almost surely. A coupling with two independent processes at time $T$ yields

$$
\begin{aligned}
& \mathbb{P}\left(L_{0}\left(u_{T+1}^{*, r}\right) \geq(-B+\Delta) T\right) \\
& \geq \mathbb{E}\left[\mathbb{1}_{\left\{\left\langle\mathbb{1}_{(-\infty,-c T]}, u_{T}^{*, r}(\cdot)\right\rangle<N\right\}} \mathbb{P}_{\mathbb{1}_{(-\infty,-c T]} u_{T}^{*, r}}(\tau \leq 1) \mathbb{P}_{\mathbb{1}_{[-c T, \infty)} u_{T}^{*, r}}\left(L_{0}\left(u_{1}\right) \geq(-B+\Delta) T\right)\right] .
\end{aligned}
$$

By [14, (2.11)], on $\left\{\left\langle\mathbb{1}_{(-\infty,-c T]}, u_{T}^{*, r}(\cdot)\right\rangle<N\right\}, \mathbb{P}_{\mathbb{1}_{(-\infty,-c T]} u_{T}^{*, r}}(\tau \leq 1) \geq \exp \left(\frac{-2 \theta N}{1-e^{-\theta}}\right)$. Note that $-B+\Delta=$ $-c-\Delta$ to further get by symmetry and domination, $\mathbb{P}_{[-c T, \infty)} u_{T}^{*, r}\left(L_{0}\left(u_{1}\right) \geq(-B+\Delta) T\right) \geq \mathbb{P}\left(R_{0}\left(u_{1}^{*, l}\right) \leq\right.$ $\Delta T)$. Hence,

$$
\mathbb{P}\left(L_{0}\left(u_{T+1}^{*, r}\right) \geq(-B+\Delta) T\right) \geq \exp \left(\frac{-2 \theta N}{1-e^{-\theta}}\right) \mathbb{P}\left(\left\langle\mathbb{1}_{(-\infty,-c T]}, u_{T}^{*, r}(\cdot)\right\rangle<N\right) \mathbb{P}\left(R_{0}\left(u_{1}^{*, r}\right) \leq \Delta T\right) .
$$

As $\lim _{T \rightarrow \infty} \mathbb{P}\left(L_{0}\left(u_{T+1}^{*, r}\right) \geq(-B+\Delta) T\right)=0$ and $\lim _{T \rightarrow \infty} \mathbb{P}\left(R_{0}\left(u_{1}^{*, r}\right) \leq \Delta T\right)=1$ by [14, Lemma 4.6] and the Markov inequality, (4.32) follows.

Corollary 4.7. For initial conditions $\psi \in \mathcal{H}^{R}, R_{0}\left(u_{T}^{(\psi)}\right) / T \rightarrow B$ for $T \rightarrow \infty$ in probability and in $\mathcal{L}^{1}$. 
Proof. As the limit is deterministic, the convergence in distribution (cf. Lemma 4.6) implies convergence in probability (cf. Grimmett and Stirzaker [8, Theorem 7.2.(4)(a)]). Use (2.3) for the negative part and Lemma 2.13 and domination for the positive part of $R_{0}\left(u_{T}^{(\psi)}\right)$ to see that the family $\left\{R_{0}\left(u_{T}^{(\psi)}\right) / T, T \geq 1\right\}$ is bounded in $\mathcal{L}^{2}$ and thus uniformly integrable (cf. [13, Corollary 6.21]). By [13, Theorem 6.25 (and Definition 6.2)], the convergence in $\mathcal{L}^{1}$ now follows from the convergence in probability of $R_{0}\left(u_{T}^{(\psi)}\right) / T$ in combination with the uniform integrability of this sequence.

Corollary 4.8. For initial conditions $\psi \in \mathcal{H}^{R}$,

$$
R_{0}\left(u_{T}^{\left(\nu^{(\psi)}\right)}\right) / T \rightarrow B \text { almost surely as } T \rightarrow \infty
$$

and $\left(0 \vee R_{0}\left(u_{T}^{\left(\nu^{(\psi)}\right)}\right)\right) / T \rightarrow B$ in $\mathcal{L}^{1}$.

Proof. By (4.1), Lemma 4.2 and Corollary 4.7,

$$
R_{0}\left(u_{T}^{\left(\nu^{(\psi)}\right)}\right) / T \rightarrow A^{\left(\nu^{(\psi)}\right)} \text { almost surely as } T \rightarrow \infty
$$

with $\mathbb{E}\left[A^{\left(\nu^{(\psi)}\right)}\right]=B$. By Proposition 4.4. $A^{\left(\nu^{(\psi)}\right)} \leq B$ almost surely. Hence, $A^{\left(\nu^{(\psi)}\right)}=B$ almost surely and the first claim follows. The second claim follows by Lemma 4.1 .

\subsection{Proof of Theorem 1.3}

The first claim and (1.17) follow from Proposition 4.4 and Corollary 4.5, Lemma 4.1 yields the $\mathcal{L}^{1}$ convergence of the positive part of the right hand side of (1.17). The third claim follows from Corollary 4.7. The forth and last claim follow from Corollary 4.8. This concludes the proof.

\section{Appendix}

\subsection{Duality}

A self duality relation in the form of [9, Subsection 1.2] respectively [14, (2.1)] holds for solutions to (1.1). For solutions with additional annihilation due to competition with a deterministic process $\beta$, see (5.1) below, a duality relation is obtained analogously. Such solutions appear for instance in the context of monotonicity-couplings, see (5.21). For existence and uniqueness of solutions to (5.1) respectively (5.2), see Theorem 1.5 .

Corollary 5.1. Let $\theta>0, T>0, \beta \in \mathcal{C}\left([0, T], \mathcal{C}_{\text {tem }}^{+}\right)$arbitrarily fixed. Let $v, z$ be independent solutions to

$$
\frac{\partial v}{\partial t}=\Delta v+(\theta-v-\beta) v+\sqrt{v} \dot{W}_{1}, \quad v(0)=v_{0}
$$

respectively

$$
\frac{\partial z}{\partial t}=\Delta z+\left(\theta-z-\beta_{T-} .\right) z+\sqrt{z} \dot{W}_{2}, \quad z(0)=z_{0}
$$

for $0 \leq t \leq T$ with $v_{0}, z_{0} \in \mathcal{C}_{\text {tem }}^{+}$and $W_{1}, W_{2}$ independent white noises. Then we have for $0 \leq s \leq T$,

$$
\mathbb{E}\left[e^{-2\langle v(T), z(0)\rangle}\right]=\mathbb{E}\left[e^{-2\langle v(s), z(T-s)\rangle}\right]=\mathbb{E}\left[e^{-2\langle v(0), z(T)\rangle}\right] .
$$

Proof. Reason as in [9, Subsection 1.2]. Let

$$
H(f, g)=2 e^{-2\langle f, g\rangle}\left(\left\langle f^{2}, g\right\rangle+\left\langle f, g^{2}\right\rangle-\langle f, \Delta g\rangle-\theta\langle f, g\rangle\right)
$$


Integration by parts yields $H(f, g)=H(g, f)$. The additional factor of 2 in the exponent results from the use of different scaling constants in the original SPDEs. Then

$$
e^{-2\left\langle v_{t}, g\right\rangle}-\int_{0}^{t} H\left(v_{s}, g\right) d s-2 \int_{0}^{t} e^{-2\left\langle v_{s}, g\right\rangle}\left\langle\beta_{s} v_{s}, g\right\rangle d s
$$

is a local martingale as well as

$$
e^{-2\left\langle z_{t}, f\right\rangle}-\int_{0}^{t} H\left(z_{s}, f\right) d s-2 \int_{0}^{t} e^{-2\left\langle z_{s}, f\right\rangle}\left\langle\beta_{T-s} z_{s}, f\right\rangle d s .
$$

As

$$
\begin{aligned}
& \frac{d}{d s} \mathbb{E}\left[e^{-2\left\langle v_{s}, z_{T-s}\right\rangle}\right] \\
& \left.\left.=\mathbb{E}\left[\left(H\left(v_{s}, z_{T-s}\right)+2 e^{-2\left\langle v_{s}, z_{T-s}\right\rangle}\left\langle\beta_{s} v_{s}, z_{T-s}\right\rangle\right)-\left(H\left(z_{T-s}, v_{s}\right)+2 e^{-2\left\langle z_{T-s}, v_{s}\right.}\right\rangle \beta_{s} z_{T-s}, v_{s}\right\rangle\right)\right]=0,
\end{aligned}
$$

the duality relation follows.

\subsection{Travelling waves for the right upper invariant measure}

We extend the construction of travelling wave solutions from solutions with compactly supported initial conditions (cf. [14]) respectively Heavyside initial data (cf. [19]) to the right upper invariant measure case. As in [14], the right marker is used to center the waves. Recall the set $\mathcal{H}^{R}$ from (1.15). The constructions extend to initial conditions $u_{0} \in \mathcal{H}^{R}$.

Let $\theta_{c}<\underline{\theta} \leq \theta \leq \bar{\theta}$ and $\nu_{T}^{*, l}=\nu_{T}^{*, l}(\theta) \in \mathcal{P}\left(\mathcal{C}_{\text {tem }}^{+}\right)$be given by

$$
\nu_{T}^{*, l}(A) \equiv T^{-1} \int_{0}^{T} \mathbb{P}\left(u_{s}^{*, l}\left(\cdot+R_{0}(s)\right) \in A\right) d s,
$$

with $\mathcal{L}\left(u_{s}^{*, l}\right)=v_{s}, s>0$ as in (1.11)-11.12). Note that by Corollaries 2.32.4, $R_{0}\left(u_{s}^{*, l}\right)$ is almost surely finite and thus $\nu_{T}^{*, l}$ is well-defined. Then the analogues of the following results of [14] hold, where constants only depend on $\theta$ through $\underline{\theta}$ and $\bar{\theta}$. Here it is important to note that the tightness-result of Lemma 5.3 from below is uniform in $\underline{\theta} \leq \theta \leq \bar{\theta}$ as well. Note that in the proof of Lemma 5.2 we use Corollaries 2.3 2.4 in place of [14, Lemma 4.8]. Also note that the constants in [19, Lemmas 3.2-3.4] hold uniform in $\underline{\theta} \leq \theta \leq \bar{\theta}$, which is easily be deduced using that $p_{t}^{\theta}(x)=e^{\theta t} p_{t}(x)$. Finally, note that the restriction to $N \in \mathbb{N}$ in [19, Lemma 3.7] and [14, Chapter 5] was only due to the fact that the sequence $\left\{\nu_{T}: T \in \mathbb{N}\right\}$ was under consideration rather than the set $\left\{\nu_{T}: T \geq 1\right\}$. As we integrate from 1 to $T$ in the proof of [19. Lemma 3.7] respectively [14, (5.2)], part of the statements below are only valid for $T>1$.

Lemma 5.2. (Analogue to [14. Lemma 4.9]) If $t>0$, then there exists $C(\underline{\theta}, \bar{\theta}, t)$ such that for all $a>0,0<s \leq t$ and $T \geq 1$,

$$
\mathbb{P}_{\nu_{T}^{*, l}}\left(\left|R_{0}(s)\right| \geq a\right) \leq \frac{C(\underline{\theta}, \bar{\theta}, t)}{a}
$$

In particular, for $0<t \leq 1$,

$$
\mathbb{P}_{\nu_{T}^{*, l}}\left(\left|R_{0}(s)\right| \geq a\right) \leq \frac{C(\underline{\theta}, \bar{\theta}) t^{1 / 4}}{a}
$$

holds.

Lemma 5.3. (Extension of [14, Lemma 5.1]) Let $\theta_{c}<\underline{\theta} \leq \theta \leq \bar{\theta}$ be arbitrarily fixed. Then the set $\left\{\nu_{T}^{*, l}(\theta): T \geq 1\right\}$ is tight. In particular, for every $\epsilon>0$ there exists a compact set $K_{\epsilon}=K_{\epsilon}(\underline{\theta}, \bar{\theta}) \subset \mathcal{C}_{\text {tem }}^{+}$ such that

$$
\inf _{\underline{\theta} \leq \theta \leq \bar{\theta}}\left(\nu_{T}^{*, l}(\theta)\right)\left(K_{\epsilon}\right) \geq 1-\epsilon \quad \text { for all } T>1 .
$$


Proof. Let $\lambda>0$ arbitrary. Then there exist $C<\infty, \gamma, \delta>0, \mu<\lambda$ and $A>0$, such that

$$
\inf _{\underline{\theta} \leq \theta \leq \bar{\theta}}\left(\nu_{T}^{*, l}(\theta)\right)\left(K(C, \delta, \gamma, \mu) \cap\left\{f \in \mathcal{C}_{\text {tem }}^{+}:\left\langle f, \phi_{1}\right\rangle \leq A\right\}\right) \geq 1-\epsilon \quad \text { for all } T>1,
$$

where $\phi_{1}(x) \equiv \exp (-|x|)$ and

$$
K(C, \delta, \gamma, \mu) \equiv\left\{f \in \mathcal{C}_{\text {tem }}^{+}:\left|f(x)-f\left(x^{\prime}\right)\right| \leq C\left|x-x^{\prime}\right|^{\gamma} e^{\mu|x|} \text { for all }\left|x-x^{\prime}\right| \leq \delta\right\} .
$$

Indeed, a look at the proof of [14, Lemma 5.1] shows that the sets under consideration, namely $K(C, \delta, \gamma, \bar{\mu})$ and $\left\{f:\left\langle f, \phi_{1}\right\rangle \leq N\right\}$ are independent of $\theta$. The bounds are derived from previous statements, where constants only depend on $\theta$ through $\underline{\theta}$ and $\bar{\theta}$.

Recall that $K \subset \mathcal{C}_{\text {tem }}^{+}$is (relatively) compact if and only if it is (relatively) compact in $\mathcal{C}_{\lambda}^{+}$for all $\lambda>0$ and that $K(C, \delta, \gamma, \mu) \cap\left\{f \in \mathcal{C}_{\text {tem }}^{+}:\left\langle f, \phi_{1}\right\rangle \leq A\right\} \equiv K(\epsilon, \lambda)$ satisfying (5.12) is compact in $\mathcal{C}_{\lambda}^{+}$(cf. [19, above (1.2)]). Now set

$$
K_{\epsilon}=\overline{\bigcap_{n \in \mathbb{N}} K\left(\epsilon 2^{-n}, 1 / n\right)}
$$

to conclude the proof of the claim.

Lemma 5.4. (Analogue to 14, Lemma 5.2]) Let $t \geq 0$ and $a, \tilde{m}>0,0<b \leq 1$ be arbitrarily fixed. Then

$$
\begin{aligned}
& \mathbb{P}_{\nu_{T}^{*, l}}\left(\left\langle u_{t}\left(\cdot+R_{0}\left(u_{t}\right)\right), \mathbb{1}_{(-2 a, \infty)}(\cdot)\right\rangle<\tilde{m}\right) \\
& \leq\left(\left(1-\frac{C_{1}(\underline{\theta}, \bar{\theta}) b^{1 / 4}}{a}\right) \vee 0\right)^{-1}\left\{\frac{T+t}{T} \frac{C_{2}(\underline{\theta}, \bar{\theta}) b^{1 / 4}}{a}+\left(1-e^{-2 \bar{\theta} \frac{\tilde{m}}{1-e^{-\underline{\theta b}}}}\right)\right\}
\end{aligned}
$$

for all $T \geq 1$.

Recall from (3.32) for $d_{0}, m_{0}>0$ the definition of $M\left(d_{0}, m_{0}\right)=\left\{f \in \mathcal{C}_{\text {tem }}^{+}\right.$: there exist $-1 / 2 \leq l_{0}<r_{0} \leq 0$ with $\left|r_{0}-l_{0}\right|=d_{0}$ such that $\left.f \geq m_{0} \mathbb{1}_{\left[l_{0}, r_{0}\right]}\right\}$.

Corollary 5.5. Let $\epsilon>0$ arbitrary. Then there exist $d_{0}=d_{0}(\epsilon), m_{0}=m_{0}(\epsilon)>0$ such that

$$
\inf _{\underline{\theta} \leq \theta \leq \bar{\theta}}\left(\nu_{T}^{*, l}(\theta)\right)\left(M\left(d_{0}, m_{0}\right)\right) \geq 1-\epsilon
$$

for all $T>1$.

Proof. In Lemma 5.4 choose $t=0$ and $a=1 / 4$. Then choose $b$ small enough and then $\tilde{m}$ small enough to obtain

$$
\inf _{\underline{\theta} \leq \theta \leq \bar{\theta}}\left(\nu_{T}^{*, l}(\theta)\right)\left(\left\{f:\left\langle f, \mathbb{1}_{(-1 / 2,0]}(\cdot)\right\rangle \geq \tilde{m}\right\}\right) \geq 1-\epsilon / 2 \quad \text { for all } T \geq 1 .
$$

By (5.12) - (5.13) for $\lambda=1$ there exist $C<\infty, \gamma, \delta>0, \mu<1$ and $A>0$, such that

$$
\inf _{\underline{\theta} \leq \theta \leq \bar{\theta}}\left(\nu_{T}^{*, l}(\theta)\right)\left(K(C, \delta, \gamma, \mu) \cap\left\{f \in \mathcal{C}_{\text {tem }}^{+}:\left\langle f, \phi_{1}\right\rangle \leq A\right\}\right) \geq 1-\epsilon / 2 \quad \text { for all } T>1 .
$$

For deterministic $f_{0} \in \mathcal{C}_{\text {tem }}^{+}$, note that if $f_{0} \in\left\{\mathcal{C}_{\text {tem }}^{+}:\left\langle f, \mathbb{1}_{[-1 / 2,0]}\right\rangle \geq \tilde{m}\right\} \cap\left\{f \in \mathcal{C}_{\text {tem }}^{+}:\left|f(x)-f\left(x^{\prime}\right)\right| \leq\right.$ $C\left|x-x^{\prime}\right|^{\gamma}$ for all $\left.x, x^{\prime} \in[-1,0],\left|x-x^{\prime}\right| \leq \delta\right\}$, then there exists $x_{0} \in[-1 / 2,0]$ such that $f\left(x_{0}\right) \geq 2 \tilde{m}$. Now use the Hölder- $\gamma$-continuity of $f_{0}$ around $x_{0}$ to obtain the existence of $d_{0}>0$ such that there exist $l_{0} \leq x_{0} \leq r_{0}, l_{0}, r_{0} \in[-1 / 2,0],\left|r_{0}-l_{0}\right|=d_{0}$ and $0<m_{0}<2 \tilde{m}$ such that $f(x) \geq m_{0}$ for all $l_{0} \leq x \leq r_{0}$.

Proposition 5.6. (Analogue to [14, Proposition 1.7]) Let $\nu_{T_{n}}^{*, l}$ be a subsequence that converges to $\nu^{*, l}$. Then $\nu^{*, l}\left(\left\{f: R_{0}(f)=0\right\}\right)=1$ and $\mathbb{P}_{\nu^{*}, l}(u(t) \not \equiv 0)=1$ for all $t \geq 0$.

Theorem 5.7. (Analogue to [14, Theorem 1.6]) Every subsequential limit of the tight set $\left\{\nu_{T}^{*, l}: T \geq 1\right\}$ yields a travelling wave solution to equation (1.1).

Remark 5.8. Recall the set $\mathcal{H}^{R}$ from (1.15). The constructions and statements from above extend to initial conditions $u_{0} \in \mathcal{H}^{R}$. Here we use Lemma 2.1 instead of (2.9). 


\subsection{Coupling techniques}

In what follows we shortly introduce the main coupling techniques and ideas that are used in this article. We start with the monotonicity-coupling from [14, Remark 2.1(i)].

Remark 5.9 (monotonicity-coupling). Let $0<\theta$ and $u_{i} \in \mathcal{C}_{\text {tem }}^{+}, i=1,2$ with $u_{1}(x) \leq u_{2}(x)$ for all $x \in \mathbb{R}$. Then there exists a coupling of solutions $u^{(i)}, i=1,2$ to (1.1) with initial conditions $u_{i}, i=1,2$ such that $u^{(1)}(t, x) \leq u^{(2)}(t, x)$ for all $t \geq 0, x \in \mathbb{R}$ almost surely. For intuition purposes, compare the construction of [16, Lemma 2.1.7]. The main idea is to write

$$
\begin{gathered}
\frac{\partial u^{(1)}}{\partial t}=\Delta u^{(1)}+\left(\theta-u^{(1)}\right) u^{(1)}+\sqrt{u^{(1)}} \dot{W}_{1}, \quad u^{(1)}(0)=u_{1}, \\
\frac{\partial v}{\partial t}=\Delta v+\left(\theta-v-2 u^{(1)}\right) v+\sqrt{v} \dot{W}_{2}, \quad v(0)=u_{2}-u_{1},
\end{gathered}
$$

where $W_{1}, W_{2}$ are independent white noises and $u^{(2)} \equiv u^{(1)}+v$ with $v(t, x) \geq 0$ for all $t \geq 0, x \in \mathbb{R}$ almost surely. $v$ is constructed (conditional on $u^{(1)}$ ) as a process with annihilation due to competition with $u^{(1)}$. Now recall [14, (1.8)] to note that

$$
\begin{aligned}
& \left\langle\int_{0}^{\cdot} \int\left|u^{(1)}(s, x)\right|^{1 / 2} \phi(x) d W_{1}(x, s)+|v(s, x)|^{1 / 2} \phi(x) d W_{2}(x, s)\right\rangle_{t}=\int_{0}^{t} \int\left(u^{(1)}(s, x)+v(s, x)\right) \phi^{2}(x) d x d s \\
& =\left\langle\int_{0}^{\cdot} \int\left|u^{(1)}(s, x)+v(s, x)\right|^{1 / 2} \phi(x) d W(x, s)\right\rangle_{t}=\left\langle\int_{0}^{\cdot} \int\left|u^{(2)}(s, x)\right|^{1 / 2} \phi(x) d W(x, s)\right\rangle_{t}
\end{aligned}
$$

for $W$ a white noise appropriately chosen.

In this article we call a $\theta$-coupling a coupling in the spirit of [16, Lemma 2.1.6]. To be more precise, use the techniques of [14, (2.2)-(2.4)] to show the following.

Remark 5.10 ( $\theta$-coupling). Let $0<\theta_{1}<\theta_{2}$. Let $u_{0} \in \mathcal{C}_{\text {tem }}^{+}$. Then there exists a coupling of solutions $u^{(i)}, i=1,2$ to (1.1) with common initial condition $u_{0}$ but different parameters $\theta_{1}$ respectively $\theta_{2}$ such that $u^{(1)}(t, x) \leq u^{(2)}(t, x)$ for all $t \geq 0, x \in \mathbb{R}$ almost surely. The main idea is to write

$$
\begin{aligned}
\frac{\partial u^{(1)}}{\partial t} & =\Delta u^{(1)}+\left(\theta_{1}-u^{(1)}\right) u^{(1)}+\sqrt{u^{(1)}} \dot{W}_{1}, \quad u^{(1)}(0)=u_{0}, \\
\frac{\partial v}{\partial t} & =\Delta v+\left(\theta_{2}-\theta_{1}\right) u^{(1)}+\left(\theta_{2}-v-2 u^{(1)}\right) v+\sqrt{v} \dot{W}_{2}, \quad v(0)=0,
\end{aligned}
$$

where $W_{1}, W_{2}$ are independent white noises and $u^{(2)} \equiv u^{(1)}+v$ with $v(t, x) \geq 0$ for all $t \geq 0, x \in \mathbb{R}$ almost surely. $v$ is constructed (conditional on $u^{(1)}$ ) as a process with annihilation due to competition with $u^{(1)}$ and an immigration-term $\left(\theta_{2}-\theta_{1}\right) u^{(1)}$.

In what follows we call a coupling with two independent processes a coupling in the spirit of [16, Lemma 2.1.7]. To be more precise, use the techniques of [14, (2.2)-(2.4)] to show the following.

Remark 5.11 (coupling with two independent processes). Let $0<\theta$. Let $u_{1}, u_{2} \in \mathcal{C}_{\text {tem }}^{+}$and $u_{0} \equiv u_{1}+u_{2}$. Then there exists a coupling of solutions $u^{(i)}, i=0,1,2$ to (1.1) with initial conditions $u_{i}, i=0,1,2$ such that $u^{(1)}$ and $u^{(2)}$ are independent and $u^{(0)}(t, x) \leq u^{(1)}(t, x)+u^{(2)}(t, x)$ for all $t \geq 0, x \in \mathbb{R}$ almost surely. The main idea is to write

$$
\begin{aligned}
\frac{\partial u^{(1)}}{\partial t} & =\Delta u^{(1)}+\left(\theta-u^{(1)}\right) u^{(1)}+\sqrt{u^{(1)}} \dot{W}_{1}, & u^{(1)}(0)=u_{1}, \\
\frac{\partial v}{\partial t} & =\Delta v+\left(\theta-v-2 u^{(1)}\right) v+\sqrt{v} \dot{W}_{2}, & v(0)=u_{2}, \\
\frac{\partial u^{(2)}}{\partial t} & =\Delta u^{(2)}+\left(\theta-u^{(2)}\right) u^{(2)}+\sqrt{u^{(2)}} \dot{W}_{2}, & u^{(2)}(0)=u_{2},
\end{aligned}
$$


where $W_{1}, W_{2}$ are independent white noises and $u^{(0)} \equiv u^{(1)}+v$ with $v(t, x) \leq u^{(2)}(t, x)$ for all $t \geq 0, x \in \mathbb{R}$ almost surely. $v$ is constructed (conditional on $\left.u^{(1)}\right)$ as a process with annihilation due to competition with $u^{(1)}$ contrary to $u^{(2)}$, where no annihilation takes place. The independence of $u^{(1)}$ and $u^{(2)}$ follows from the independence of the white noises $W_{1}, W_{2}$.

An immigration-coupling is constructed similarly to a $\theta$-coupling, where the immigration-term only depends on an outside source.

Remark 5.12 (immigration-coupling). Let $\alpha_{1}, \alpha_{2}-\alpha_{1} \in \mathcal{C}\left([0, \infty), \mathcal{C}_{\text {tem }}^{+}\right)$. Let $u_{0} \in \mathcal{C}_{\text {tem }}^{+}$. Then there exists a coupling of solutions $u^{(i)}, i=1,2$ solving

$$
\frac{\partial u^{(i)}}{\partial t}=\Delta u^{(i)}+\alpha_{i}+\left(\theta-u^{(i)}\right) u^{(i)}+\sqrt{u^{(i)}} \dot{W}_{i}, \quad u^{(i)}(0)=u_{0}, \quad i=1,2
$$

with $W_{1}, W_{2}$ two independent white noises, such that $u^{(1)}(t, x) \leq u^{(2)}(t, x)$ for all $t \geq 0, x \in \mathbb{R}$ almost surely. The main idea is to write $u^{(2)} \equiv u^{(1)}+v$ with $v \geq 0$ satisfying

$$
\frac{\partial v}{\partial t}=\Delta v+\left(\alpha_{2}-\alpha_{1}\right)+\left(\theta-v-2 u^{(1)}\right) v+\sqrt{v} \dot{W}_{2}, \quad v(0)=0
$$

$v$ is constructed (conditional on $\left.u^{(1)}\right)$ as a process with annihilation due to competition with $u^{(1)}$ and an immigration-term $\alpha_{2}-\alpha_{1}$.

Note that conditional on $u^{(1)} \in \mathcal{C}\left([0, \infty), \mathcal{C}_{t e m}^{+}\right)$all the processes $(v(t))_{t \geq 0}$ fit into the framework of [19, (2.4)] and are as such non-negative. The final coupling we present is of a different flavor. It is based on the approximation of solutions to (1.1) with initial conditions $u_{0} \in \mathcal{C}_{\text {tem }}^{+}$by means of densities of rescaled long-range contact processes, see [17, Theorem 1] for the convergence result. Note that the parameter $\theta_{c}$ in [17] denotes an arbitrary $\theta>0$ and does not have any relation to the critical parameter $\theta_{c}$ of the present article. Changes in constants are

We use the construction of an approximating particle system $\left(\xi_{t}^{n}\left(f_{0}\right)\right)_{t \geq 0}$ for $n \in \mathbb{N}$ resulting in a solution to (1.1) with initial condition $f_{0} \in \mathcal{C}_{\text {tem }}^{+}$from [17]. The dynamics are modeled by means of i.i.d. Poisson processes given at the beginning of Section 2. Their rates depend in a monotone way on the parameter $\theta$. Initial conditions $f_{0}$ get approximated by approximate densities $A_{c}\left(\xi_{0}^{n}\left(f_{0}\right)\right), n \in \mathbb{N}$, compare the definition preceding Theorem 1 . The approximate densities $\left(A_{c}\left(\xi_{t}^{n}\left(f_{0}\right)\right)_{t \geq 0}\right.$ converge to a solution to (1.1) with initial condition $f_{0}$.

For the next lemma, recall the definition of $v_{T}=v_{T}(\theta)$ from [14, Remark 2.8]. Note in particular the use of the non-decreasing sequence $\zeta_{N} \in \mathcal{C}_{\text {tem }}^{+}, N \in \mathbb{N}$.

Lemma 5.13 ( $\theta$-*-coupling). Let $0<\theta_{1}<\theta_{2}$ and $T>0$ be arbitrarily fixed. There exists a coupling of two processes $\left(u_{T+t}^{*, l}\left(\theta_{i}\right)\right)_{t \geq 0}, i=1,2$ such that $\mathcal{L}\left(\left(u_{T+t}^{*, l}\left(\theta_{i}\right)\right)_{t \geq 0}\right)=\mathbb{P}_{v_{T}\left(\theta_{i}\right)}, i=1,2$. Moreover,

$$
\left(u_{T+t}^{*, l}\left(\theta_{1}\right)\right)(x) \leq\left(u_{T+t}^{*, l}\left(\theta_{2}\right)\right)(x) \text { for all } x \in \mathbb{R}, t \geq 0 \text { a.s. }
$$

This result also holds for a finite number of $0<\theta_{1}<\cdots<\theta_{m}, m \in \mathbb{N}$.

This coupling relies on two properties of the processes involved. Firstly, we use the monotonicity of the respective solutions resulting from $\theta_{1}<\theta_{2}$ for each initial condition $\zeta_{N}, N \in \mathbb{N}$, secondly, for $\theta_{i}$ fixed, we use the construction of $\left(u_{T+t}^{*, l}\left(\theta_{i}\right)\right)_{t \geq 0}$ by means of a non-decreasing sequence $\left(u_{T+t}^{\left(\zeta_{N}\right)}\left(\theta_{i}\right)\right)_{t \geq 0}, N \in \mathbb{N}$ as in [14, Remark 2.8]. Unfortunately, we could not make the constructions from above work to integrate these two steps into one. Thus we had to make use of the approximation by discrete particle systems, where at least the motivation for the veracity of the above result should be easily accessible to the reader. 
Proof. The dynamics of the $n^{\text {th }}$ approximation for $\theta_{i}, i=1,2$ use the same set of i.i.d. Poisson processes for death-events. For birth-events, consider i.i.d. Poisson processes

$$
\begin{aligned}
& \left(P_{t}(x, y): x, y \in n^{-2} \mathbb{Z}, x \text { neigbor of } y\right) \text { with rate }\left(2 c_{1} n^{3 / 2}\right)^{-1}\left(n+\theta_{1}\right), \\
& \left(Q_{t}(x, y): x, y \in n^{-2} \mathbb{Z}, x \text { neighbor of } y\right) \text { with rate }\left(2 c_{1} n^{3 / 2}\right)^{-1}\left(\theta_{2}-\theta_{1}\right),
\end{aligned}
$$

where $c_{1}(n) \rightarrow 1$ as $n \rightarrow \infty$. For the $\theta_{1}$-system, at a jump of $P_{t}(x, y)$, if the site $x$ is occupied, there is a birth and the site $y$, if vacant, becomes occupied (cf. beginning of [17, Section 2]). In our coupling, for the $\theta_{2}$-system, at a jump of $P_{t}(x, y)$ or $Q_{t}(x, y)$ the same holds. Note that $\left(P_{t}(x, y)+Q_{t}(x, y): x, y \in\right.$ $n^{-2} \mathbb{Z}, x$ neighbor of $\left.y\right)$ is a family of i.i.d. Poisson processes with rate $\left(2 c_{1} n^{3 / 2}\right)^{-1}\left(n+\theta_{2}\right)$. As a result, given the same initial configurations, the $\theta_{2}$-system dominates the $\theta_{1}$-system.

Additionally, we construct a set of initial conditions $\left(\xi_{0}^{n}\left(\zeta_{N}\right): N \in \mathbb{N}\right)$ of the $n^{\text {th }}$ approximating particle systems as follows below. They are the same for the $\theta_{1}$ - and $\theta_{2}$-system. After linear interpolation in space, $A_{c}\left(\xi_{0}^{n}\left(\zeta_{N}\right)\right)$ converges in $\mathcal{C}_{\text {tem }}^{+}$to $\zeta_{N}$ for $n \rightarrow \infty$ for all $N \in \mathbb{N}$ and (use that the sequence $\left(\zeta_{N}\right)_{N \in \mathbb{N}}$ is non-decreasing), $\xi_{0}^{n}\left(\zeta_{N_{1}}\right) \leq \xi_{0}^{n}\left(\zeta_{N_{2}}\right)$ for $N_{1} \leq N_{2}$. By [17, Theorem 1], the approximating densities $\left(A_{c}\left(\xi_{t}^{n}\left(\zeta_{N}\right)\right)\left(\theta_{i}\right)\right)_{t \geq 0}, i=1,2$ converge in distribution for $n \rightarrow \infty$ to continuous solutions $\left(u_{t}^{\left(\zeta_{N}\right)}\left(\theta_{i}\right)\right)_{t \geq 0}, i=$ 1,2 of (1.1) with initial conditions $\zeta_{N} \in \mathcal{C}_{\text {tem }}^{+}$. By construction,

$$
A_{c}\left(\xi_{t}^{n}\left(\zeta_{N_{1}}\right)\right)\left(\theta_{i}\right) \leq A_{c}\left(\xi_{t}^{n}\left(\zeta_{N_{2}}\right)\right)\left(\theta_{j}\right)
$$

for all $t \geq 0, N_{1} \leq N_{2}, \theta_{i} \leq \theta_{j}, i, j \in\{1,2\}$.

For $n \in \mathbb{N}$ fixed, use the following coupling to obtain $n^{t h}$-approximations $\left(\xi_{0}^{n}\left(\zeta_{N}\right): N \in \mathbb{N}\right)$ for a family of initial conditions $\left(\zeta_{N}\right)_{N \in \mathbb{N}}$ as in [14, Remark 2.8]. Assume without loss of generality that for all $N \in \mathbb{N}$, $\zeta_{N} \in \mathcal{C}_{\text {tem }}^{+}$is a bounded continuously differentiable function with bounded first derivatives. To construct the initial conditions of the $n^{t h}$ approximating particle system recall that each site $z \in n^{-2} \mathbb{Z}$ has $2 c_{1} n^{3 / 2}$ neighbors (including $z$ ) and for $f_{0} \in \mathcal{C}_{\text {tem }}^{+}, A_{c}\left(\xi_{0}^{n}\left(f_{0}\right)\right)(z)=\left(2 c_{1} n^{1 / 2}\right)^{-1} \sum_{y \text { neighbor of } z}\left(\xi_{0}^{n}\left(f_{0}\right)\right)(y)$. For $z \in n^{-2} \mathbb{Z}$, let

$$
\left(\xi_{0}^{n}\left(f_{0}\right)\right)(z)= \begin{cases}1, & \exists k \in \mathbb{Z}: z \in\left\{\frac{k \cdot 2 c_{1} n^{3 / 2}}{n^{2}}, \frac{k \cdot 2 c_{1} n^{3 / 2}+1}{n^{2}}, \ldots, \frac{k \cdot 2 c_{1} n^{3 / 2}+\left\lfloor 2 c_{1} n^{1 / 2} f_{0}\left(k c_{1} n^{-1 / 2}\right)\right\rfloor}{n^{2}}\right\} \\ 0, \quad \text { otherwise. }\end{cases}
$$

Then $A_{c}\left(\xi_{0}^{n}\left(\zeta_{N}\right)\right)$, after linear interpolation in space, converges in $\mathcal{C}_{\text {tem }}^{+}$to $\zeta_{N}$ for $n \rightarrow \infty$ for all $N \in \mathbb{N}$ and by construction, (5.30) is fulfilled.

Assume without loss of generality that $\tilde{d}$ is such that $\left(\mathcal{D}\left([0, \infty), \mathcal{C}_{\text {tem }}^{+}\right), \tilde{d}\right)$ is a Polish space (recall $d$ from below [14, (1.6)] and cf. Ethier and Kurtz [6, Theorem III.5.6]). We have

$$
\begin{aligned}
& \left(A_{c}\left(\xi^{n}\left(\zeta_{N}\right)\right)\left(\theta_{1}\right), A_{c}\left(\xi^{n}\left(\zeta_{N}\right)\right)\left(\theta_{2}\right), A_{c}\left(\xi^{n}\left(\zeta_{N}\right)\right)\left(\theta_{2}\right)-A_{c}\left(\xi^{n}\left(\zeta_{N}\right)\right)\left(\theta_{1}\right),\right. \\
& \left.A_{c}\left(\xi^{n}\left(\zeta_{N+1}\right)\right)\left(\theta_{2}\right)-A_{c}\left(\xi^{n}\left(\zeta_{N}\right)\right)\left(\theta_{2}\right), A_{c}\left(\xi^{n}\left(\zeta_{N+1}\right)\right)\left(\theta_{1}\right)-A_{c}\left(\xi^{n}\left(\zeta_{N}\right)\right)\left(\theta_{1}\right)\right)_{N \in \mathbb{N}} \\
\equiv & \left(A_{n}\left(N, \theta_{1}\right), A_{n}\left(N, \theta_{2}\right), A_{n}(N, \Delta \theta), A_{n}\left(\Delta N, \theta_{1}\right), A_{n}\left(\Delta N, \theta_{2}\right)\right)_{N \in \mathbb{N}} \\
\equiv & A_{n} \in X \equiv\left(\left(\mathcal{D}\left([0, \infty), \mathcal{C}_{t e m}^{+}\right)\right)^{5}\right)^{\mathbb{N}} .
\end{aligned}
$$

Then $X$ is a Polish space as well if we equip it with the metric $\rho(\bar{f}, \bar{g}) \equiv \sum_{i \in \mathbb{N}} 2^{-i}\left(\sum_{j=1,2,3,4,5} \tilde{d}\left(f_{i 1}, g_{i 1}\right)\right) \wedge$ 1 where $\bar{f}=\left(f_{i j}\right)_{i \in \mathbb{N}, j=1,2,3,4,5}, \bar{g}=\left(g_{i j}\right)_{i \in \mathbb{N}, j=1,2,3,4,5} \in X, f_{i j}, g_{i j} \in \mathcal{D}\left([0, \infty), \mathcal{C}_{t e m}^{+}\right)$. Reason as in Jacod and Shiryaev [12, Corollary VI.3.33], to see that the convergence in distribution of the sequences $\left(A_{c}\left(\xi^{n}\left(\zeta_{N}\right)\right)\left(\theta_{j}\right)\right)_{n \in \mathbb{N}}$ for $j \in\{1,2\}, N \in \mathbb{N}$ fixed and $n \rightarrow \infty$ to a continuous (in $t$ ) limit implies the convergence of

$$
\left(A_{n}\left(N, \theta_{1}\right), A_{n}\left(N, \theta_{2}\right), A_{n}\left(N, \Delta \theta_{1}\right), A_{n}\left(\Delta N, \theta_{1}\right), A_{n}\left(\Delta N, \theta_{2}\right)\right)_{n \in \mathbb{N}}
$$


to

$$
\left(u .^{\left(\zeta_{N}\right)}\left(\theta_{1}\right), u \cdot^{\left(\zeta_{N}\right)}\left(\theta_{2}\right), u .^{\left(\zeta_{N}\right)}\left(\theta_{2}\right)-u .^{\left(\zeta_{N}\right)}\left(\theta_{1}\right), u .^{\left(\zeta_{N+1}\right)}\left(\theta_{1}\right)-u \cdot^{\left(\zeta_{N}\right)}\left(\theta_{1}\right), u .^{\left(\zeta_{N+1}\right)}\left(\theta_{2}\right)-u .^{\left(\zeta_{N}\right)}\left(\theta_{2}\right)\right)
$$

By the definition of $\rho(\bar{f}, \bar{g})$, we can choose a subsequence such that $\left(A_{n_{k}}\right)_{k \in \mathbb{N}}$ converges in $X$. Note in particular that the marginal distributions of every subsequential limit are given by their respective one-dimensional limits.

Fix this convergent subsequence. Now apply Skorokhod's theorem (cf. [6, Theorem III.1.8]) to obtain that after possibly changing to another probability space, this convergence becomes almost sure convergence, that is

$$
\begin{aligned}
& \left(A_{n_{k}}\left(N, \theta_{1}\right), A_{n_{k}}\left(N, \theta_{2}\right), A_{n_{k}}\left(N, \Delta \theta_{1}\right), A_{n_{k}}\left(\Delta N, \theta_{1}\right), A_{n_{k}}\left(\Delta N, \theta_{2}\right)\right)_{N \in \mathbb{N}} \\
& \rightarrow\left(\left(u^{\left(\zeta_{N}\right)}\left(\theta_{1}\right), u \cdot^{\left(\zeta_{N}\right)}\left(\theta_{2}\right), u \cdot^{\left(\zeta_{N}\right)}\left(\theta_{2}\right)-u \cdot^{\left(\zeta_{N}\right)}\left(\theta_{1}\right),\right.\right. \\
& \left.\quad u .^{\left(\zeta_{N+1}\right)}\left(\theta_{1}\right)-u .^{\left(\zeta_{N}\right)}\left(\theta_{1}\right), u \cdot^{\left(\zeta_{N+1}\right)}\left(\theta_{2}\right)-u \cdot^{\left(\zeta_{N}\right)}\left(\theta_{2}\right)\right)_{N \in \mathbb{N}} \in X \text { a.s. for } k \rightarrow \infty
\end{aligned}
$$

where $u^{\left(\zeta_{N}\right)}\left(\theta_{i}\right)$ solves (1.1) with initial condition $\zeta_{N}$ and parameter $\theta_{i}$ and

$$
u^{\left(\zeta_{N_{1}}\right)}\left(\theta_{j_{1}}\right) \leq u^{\left(\zeta_{N_{2}}\right)}\left(\theta_{j_{2}}\right) \text { for all } N_{1} \leq N_{2}, j_{1} \leq j_{2} \text { a.s. }
$$

by (5.35) in combination with the definition of $X$.

Fix $T>0$. Let $u_{T+t}^{*, l}\left(\theta_{i}\right)=\uparrow \lim _{N \rightarrow \infty} u_{T+t}^{\left(\zeta_{N}\right)}\left(\theta_{i}\right), i=1,2, t \geq 0$. Reason as in [14, Corollary 2.6] to conclude that $\mathcal{L}\left(\left(u_{T+t}^{*, l}\left(\theta_{i}\right)\right)_{t \geq 0}=\mathbb{P}_{v_{T}}\right.$. From (5.36), (5.28) follows.

Acknowledgements. This work was funded by the Deutsche Forschungsgemeinschaft (DFG, German Research Foundation) - Projectnumber 393092071.

\section{References}

[1] D.G. Aronson and H.F. Weinberger. Nonlinear diffusion in population genetics, combustion, and nerve pulse propagation. In Partial differential equations and related topics (Program, Tulane Univ., New Orleans, La., 1974), volume 446 of Lecture Notes in Math., pages 5-49. Springer, Berlin, 1975.

[2] M. Bessonov and R. Durrett. Phase transitions for a planar quadratic contact process. Adv. in Appl. Math., 87:82-107, 2017.

[3] R. Durrett. On the growth of one-dimensional contact processes. Ann. Probab., 8(5):890-907, 1980.

[4] R. Durrett. Oriented percolation in two dimensions. Ann. Probab., 12(4):999-1040, 1984.

[5] R. Durrett. Ten lectures on particle systems. In Lectures on Probability Theory (Saint-Flour, 1993), volume 1608 of Lecture Notes in Math., pages 97-201. Springer, Berlin, 1995.

[6] S.N. Ethier and T.G. Kurtz. Markov Processes: Characterization and Convergence. Wiley and Sons, Inc., Hoboken, New Jersey, 2005.

[7] D. Griffeath. The basic contact processes. Stochastic Process. Appl., 11(2):151-185, 1981.

[8] G.R. Grimmett and D.R. Stirzaker. Probability and random processes. Oxford University Press, New York, third edition, 2001.

[9] P. Horridge and R. Tribe. On stationary distributions for the KPP equation with branching noise. Ann. Inst. H. Poincaré Probab. Statist., 40(6):759-770, 2004. 
[10] I. Iscoe. A weighted occupation time for a class of measure-valued branching processes. Probab. Theory Related Fields, 71(1):85-116, 1986.

[11] I. Iscoe. On the supports of measure-valued critical branching Brownian motion. Ann. Probab., 16(1):200-221, 1988.

[12] J. Jacod and A.N. Shiryaev. Limit Theorems for Stochastic Processes, volume 288 of Grundlehren Math. Wiss. Springer, Berlin, second edition edition, 2003.

[13] A. Klenke. Probability Theory. Universitext. Springer, London, second edition, 2014.

[14] S. Kliem. Travelling wave solutions to the KPP equation with branching noise arising from initial conditions with compact support. Stochastic Process. Appl., 127(2):385-418, 2017.

[15] T.M. Liggett. Stochastic interacting systems: contact, voter and exclusion processes. Springer, Berlin Heidelberg New York, 1999.

[16] C. Mueller and R. Tribe. A phase transition for a stochastic PDE related to the contact process. Probab. Theory Related Fields, 100:131-156, 1994.

[17] C. Mueller and R. Tribe. Stochastic p.d.e.'s arising from the long range contact and long range voter processes. Probab. Theory Related Fields, 102:519-545, 1995.

[18] E.A. Perkins. Dawson-Watanabe superprocesses and measure-valued diffusions, volume 1781 of Lecture Notes in Math. Springer, Berlin, lectures on probability theory and statistics (saint-flour, 1999) edition, 2002.

[19] R. Tribe. A travelling wave solution to the Kolmogorov equation with noise. Stochastics Stochastics Rep., 56:317-340, 1996. 